TRANSACTIONS OF THE

AMERICAN MATHEMATICAL SOCIETY

Volume 365, Number 1, January 2013, Pages 251-301

S 0002-9947(2012)05567-X

Article electronically published on July 11, 2012

\title{
STABILITY OF PULSE SOLUTIONS FOR THE DISCRETE FITZHUGH-NAGUMO SYSTEM
}

\author{
H. J. HUPKES AND B. SANDSTEDE
}

\begin{abstract}
We show that the fast travelling pulses of the discrete FitzHughNagumo system in the weak-recovery regime are nonlinearly stable. The spectral conditions that need to be verified involve linear operators that are associated to functional differential equations of mixed type. Such equations are ill-posed and do not admit a semi-flow, which precludes the use of standard Evans-function techniques. Instead, we construct the potential eigenfunctions directly by using exponential dichotomies, Fredholm techniques and an infinitedimensional version of the Exchange Lemma.
\end{abstract}

\section{INTRODUCTION}

In this paper we consider the stability of travelling pulse solutions to the discrete FitzHugh-Nagumo system

$$
\begin{aligned}
& \dot{u}_{j}(t)=\alpha\left[u_{j+1}(t)-2 u_{j}(t)+u_{j-1}(t)\right]+g\left(u_{j}(t)\right)-w_{j}(t), \\
& \dot{w}_{j}(t)=\epsilon\left(u_{j}(t)-\gamma w_{j}(t)\right),
\end{aligned}
$$

where $j \in \mathbb{Z}$ and $u_{j}, w_{j} \in \mathbb{R}$. The nonlinearity $g(u)=g(u ; a)$ that we use throughout this paper is given by the cubic polynomial

$$
g(u ; a)=u(1-u)(u-a)
$$

for some $0<a<\frac{1}{2}$, but our techniques work equally well for the general class of bistable nonlinearities considered in HS10. Throughout this paper we assume that $\alpha>0$ and $0<\gamma<4(a-1)^{-2}$, which ensures that the origin is the only $j$-independent equilibrium for (1.1) when $\epsilon>0$. We also need the following condition, which in view of [MP99b, Thm. 2.6] can be satisfied by picking the detuning parameter $a>0$ to be sufficiently small.

Hypothesis (H). The lattice system (1.1) with $\epsilon=0$ admits a travelling wave solution

$$
\left(u_{j}, w_{j}\right)(t)=\left(q_{f}\left(j+c_{*} t\right), 0\right)
$$

for some speed $c_{*}>0$ and profile $q_{f} \in C^{1}(\mathbb{R}, \mathbb{R})$ that satisfies the limits

$$
\lim _{\xi \rightarrow-\infty} q_{f}(\xi)=0 \quad \text { and } \quad \lim _{\xi \rightarrow \infty} q_{f}(\xi)=1 .
$$

Inserting the travelling wave Ansatz

$$
\left(u_{j}, w_{j}\right)(t)=(\bar{u}, \bar{w})(j+c t)
$$

Received by the editors May 6, 2010 and, in revised form, February 1, 2011.

2010 Mathematics Subject Classification. Primary 34A33, 34K26, 34D35, 34K08.

Key words and phrases. Discrete FitzHugh-Nagumo system, travelling pulses, stability, singular perturbation theory, lattice differential equations, functional differential equations.

(C) 2012 American Mathematical Society 
into (1.1) leads to the singularly perturbed functional differential equation of mixed type (MFDE)

$$
\begin{aligned}
& c \bar{u}^{\prime}(\xi)=\alpha[\bar{u}(\xi+1)-2 \bar{u}(\xi)+\bar{u}(\xi-1)]+g(\bar{u}(\xi))-\bar{w}(\xi), \\
& c \bar{w}^{\prime}(\xi)=\epsilon(\bar{u}(\xi)-\gamma \bar{w}(\xi)) .
\end{aligned}
$$

Our assumption on $\gamma$ guarantees that $(0,0)$ is the only equilibrium of (1.6) when $\epsilon>0$. However, when $\epsilon=0$ this system admits an entire manifold $\mathcal{M}=\{(u, g(u))$ : $u \in \mathbb{R}\}$ of equilibria. As illustrated in Figure 1, we pick two submanifolds $\mathcal{M}_{L}$ and $\mathcal{M}_{R}$ that lie to the left of the first knee and to the right of the second knee of $g$. The pair $\left(q_{f}, 0\right)$ solves (1.6) at $\epsilon=0$ and connects $(0,0) \in \mathcal{M}_{L}$ to $(1,0) \in \mathcal{M}_{R}$. By exploiting the mirror symmetry of the cubic polynomial $g$, one can see that there exists $w_{*}>0$ such that this equation also admits a solution $\left(q_{b}, w_{*}\right)$ that connects $\mathcal{M}_{R}$ to $\mathcal{M}_{L}$, i.e.,

$$
\left(\lim _{\xi \rightarrow-\infty} q_{b}(\xi), w_{*}\right) \in \mathcal{M}_{R} \quad \text { and } \quad\left(\lim _{\xi \rightarrow \infty} q_{b}(\xi), w_{*}\right) \in \mathcal{M}_{L} .
$$

Let us write $\Gamma_{0}$ for the singular homoclinic orbit that arises by combining these two orbits with the segments of $\mathcal{M}_{R}$ and $\mathcal{M}_{L}$ that connect $w=0$ to $w=w_{*}$; see Figure 1. In our previous paper [HS10], we constructed a branch of fast pulse solutions to (1.6) that bifurcate off $\Gamma_{0}$ as $\epsilon$ moves away from zero and wind around this singular homoclinic orbit exactly once.

Theorem 1.1 (See HS10). Consider the discrete FitzHugh-Nagumo equation (1.1) with the nonlinearity (1.2) and suppose that $(H)$ is satisfied. Then for every sufficiently small $\epsilon>0$, there exist functions $\bar{u}(\epsilon), \bar{w}(\epsilon) \in C^{1}(\mathbb{R}, \mathbb{R})$ and a wave speed $c(\epsilon)>0$ that depends continuously on $\epsilon>0$ with $c(0)=c_{*}$ such that

$$
\left(u_{j}, w_{j}\right)(t)=(\bar{u}(\epsilon), \bar{w}(\epsilon))(j+c(\epsilon) t)
$$

is a travelling pulse solution of (1.1) that winds around $\Gamma_{0}$ once and satisfies the limits

$$
\lim _{\xi \rightarrow \pm \infty}(\bar{u}(\epsilon), \bar{w}(\epsilon))(\xi)=(0,0) .
$$

Up to temporal translations, this pulse is uniqu@ among solutions with these properties.

The main result that we set out to prove in this paper states that the fast pulses described in Theorem 1.1 are nonlinearly stable. Of course, we have to allow for asymptotic phase shifts due to the temporal invariance of (1.1). A precise version of this result will be stated in $₫ 2$,

Theorem 1.2. Consider the setting of Theorem 1.1. For every sufficiently small $\epsilon>0$, the travelling pulse solution

$$
\left(u_{j}, w_{j}\right)(t)=(\bar{u}(\epsilon), \bar{w}(\epsilon))(j+c(\epsilon) t)
$$

is asymptotically stable with an asymptotic phase shift: each solution to the system (1.1) that is sufficiently close to this pulse at $t=0$ will remain in a small neighbourhood of the pulse for all $t>0$ and converge to some temporal translate of the pulse as $t \rightarrow \infty$.

\footnotetext{
${ }^{1}$ We refer to HS10 for a definition of the winding number and a precise version of the uniqueness claim.
} 


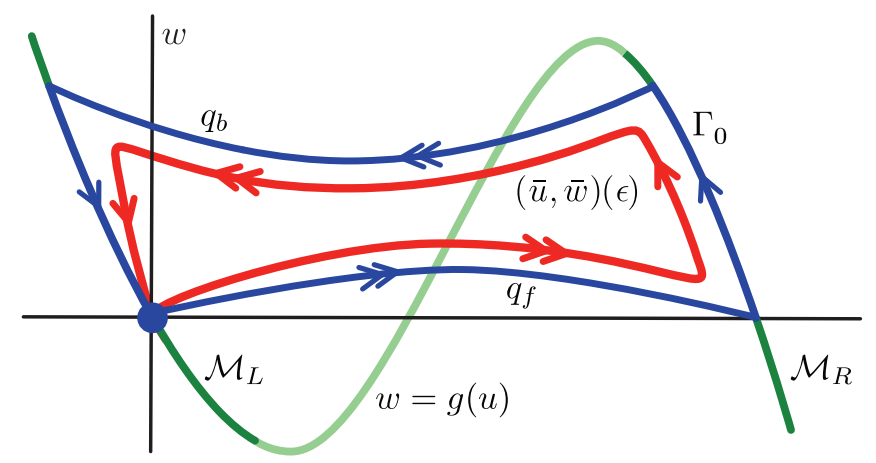

FiguRE 1. Shown is the singular homoclinic orbit $\Gamma_{0}$ comprised of the front $\left(q_{f}, 0\right)$, the back $\left(q_{b}, w_{*}\right)$ and segments of $\mathcal{M}_{L}$ and $\mathcal{M}_{R}$. Also shown are the pulse solutions $(\bar{u}, \bar{w})(\epsilon)$ described in Theorem 1.1 .

Before we outline the ideas behind the proof of our stability result, we briefly recall the motivation for studying the discrete FitzHugh-Nagumo equation and comment on related work.

One natural way for the discrete system (1.1) to arise occurs when $\alpha=h^{-2} \gg 1$, in which case we may think of (1.1) as the spatial finite-difference discretization of the FitzHugh-Nagumo PDE

$$
\begin{aligned}
& u_{t}=u_{x x}+g(u)-w, \\
& w_{t}=\epsilon(u-\gamma w),
\end{aligned}
$$

on a grid with node distance $h$. This PDE has been studied extensively in the literature, serving as a tractable simplification of the Hodgkin-Huxley equations that are widely used to model the propagation of signals through myelinated nerve fibers [HHK52]. However, from a modelling point of view it turns out to be more natural to use the discrete system (1.1) and avoid the $h \rightarrow 0$ limit that leads to the PDE (1.11). This is related to the fact that a nerve axon is almost entirely surrounded by an insulating myeline coating, which admits small regularly-spaced gaps that are referred to as the nodes of Ranvier [Ran78]. The insulation induced by the myeline causes excitations of the nerve at these nodes to effectively jump from one node to the next Lil25, HS49, leading to a system of the form (1.1) to describe the electrochemical properties at the $j$-th node of Ranvier KS98. Many other natural systems admit such an intrinsic discrete structure. As a consequence, models involving lattice differential equations (LDEs) have appeared in a wide range of scientific disciplines, including chemical reaction theory [EN93, LE92, material science [BC99, Cah60] and image processing and pattern recognition CR93.

The search for travelling pulse solutions to the PDE (1.11) has led to the development of many new mathematical techniques. The existence of a branch of fast 2 pulses for (1.11) that bifurcates from the singular orbit $\Gamma_{0}$ as in Theorem 1.1 was first established by Carpenter Car77] and Hastings Has76], who obtained their results independently using classical singular perturbation theory Car77, and the Conley index Has76. A more streamlined approach was developed by Jones

\footnotetext{
${ }^{2}$ The term 'fast' here refers to the fact that 1.11) also admits a branch of slow pulses with speed $c=O(\sqrt{\epsilon})$.
} 


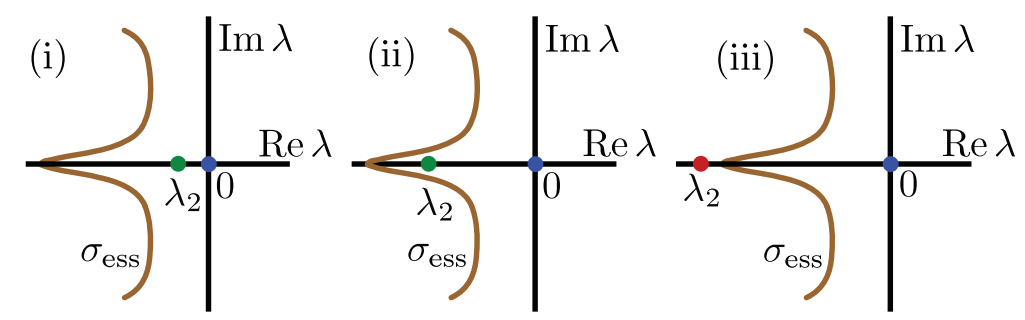

Figure 2. The Floquet spectrum of the linearization of (1.1) about the fast pulse is illustrated for $\epsilon>0$ : the spectrum near the origin consists of a curve of essential spectrum, a simple eigenvalue at the origin, and at most one additional simple stable eigenvalue $\lambda_{2}$ near zero. Depending on the values of $a$ and $\gamma, \lambda_{2}$ is an eigenvalue (panels (i) and (ii)) or a resonance pole (panel (iii)), and $\lambda_{2}$ may lie to the right or left of the rightmost elements of the essential spectrum. We refer to $\$ 7$ for further details.

and coworkers [JKL91, who used a geometrical construction called the Exchange Lemma to exhibit these fast pulses as intersections of $\mathcal{W}^{u}(0)$ and $\mathcal{W}^{s}\left(\mathcal{M}_{L}\right)$, thereby also obtaining their local uniqueness. Jones proved in [Jon84 that these fast pulses are stable with respect to the dynamics of the PDE (1.11). His arguments were significantly simplified by Yanagida Yan85, but both authors essentially used shooting arguments to construct and analyze an Evans function Eva75] that encodes the stability properties of the pulses.

In contrast, very few rigorous results have appeared in the literature for the discrete FitzHugh-Nagumo equation (1.1). Previous work either replaced the cubic polynomial $g$ by specially tailored nonlinearities [Ton03, EVV05, CH99] or employed formal asymptotic arguments [Car05a, Car05b, CB02]. On the other hand, numerical simulations give strong evidence for the existence of stable pulse solutions of (1.1), and we refer in particular to the work [CB02] of Carpio and Bonilla. Much more is known for the Nagumo lattice system

$$
\dot{u}_{j}=\alpha\left[u_{j+1}-2 u_{j}+u_{j-1}\right]+g\left(u_{j}\right),
$$

which arises when considering (1.1) for $\epsilon=0$ with $w_{j} \equiv 0$. In particular, results concerning the existence of the front solution $q_{f}$ mentioned in $(\mathrm{H})$ can be found in Kee87, HZ93, MP99b. The first result pertaining to the stability of the front $q_{f}$ with respect to the dynamics of (1.12) was obtained by Zinner [Zin91], using methods based upon the comparison principles developed in [Kee87.

We now comment on the proof of Theorem 1.2 for the FitzHugh-Nagumo system, for which comparison principles are not available. The key step of our stability analysis is concerned with the spectral stability of the pulse solution, which we address by investigating the spectrum of the linearization of the travelling wave equation (1.6) about the pulse. The corresponding eigenvalue problem is given by (1.13)

$$
\begin{aligned}
& c(\epsilon) u^{\prime}(\xi)=\alpha[u(\xi-1)+u(\xi+1)-2 u(\xi)]+g^{\prime}(\bar{u}(\epsilon)(\xi)) u(\xi)-w(\xi)-\lambda u(\xi), \\
& c(\epsilon) w^{\prime}(\xi)=\epsilon(u(\xi)-\gamma w(\xi))-\lambda w(\xi) .
\end{aligned}
$$

We will see that the essential spectrum associated to (1.13) lies at a distance $O(\epsilon)$ to the left of the imaginary axis. Furthermore, the translational invariance of (1.6) 
implies that there is one eigenvalue at $\lambda=0$. The pulse is constructed by gluing a stable front and a stable back together, each of which have a simple eigenvalue at the origin. Thus, we can think of the pulse as a bound state of a front and a back, and we may therefore expect to find two eigenvalues near the origin that come from the eigenvalues of the front and back at the origin. One of these will be the translational eigenvalue of the pulse that we already discussed, while the second eigenvalue can be thought of as an interaction eigenvalue of the front and the back. Our task is to make this intuitive picture precise by showing that (1.13) can have at most one nonzero eigenvalue near the origin, and that this eigenvalue lies in the left half-plane. Since the initial-value problem associated with the linear MFDE (1.13) is ill-posed [Rus89], we cannot readily use Evans function techniques to track eigenvalues. Instead, we use exponential dichotomies to explicitly construct potential eigenfunctions of (1.13) for $(\lambda, \epsilon)$ near $(0,0)$. These potential eigenfunctions are continuous up to two jumps, and requiring that these jumps vanish yields a two-dimensional set of bifurcation equations that depend on $\lambda$ and $\epsilon$ : any solution $\lambda$ of this system corresponds to an eigenvalue or a resonance pole (the eigenfunction associated with the latter may not be bounded). We show that the bifurcation equations are given in terms of appropriate Melnikov integrals, whose sign is known, and use this information to calculate the leading-order expansion of $\lambda_{2}$ in terms of $\epsilon$. In particular, in contrast to the Evans function techniques used in [Jon84, Yan85, our approach therefore gives the approximate location of the second eigenvalue $\lambda_{2}$. The dynamics of (1.1) near the pulse $(\bar{u}, \bar{w})(\epsilon)$ will depend on the location of $\lambda_{2}$ relative to the imaginary axis and the essential spectrum. In 97 , we provide numerical evidence that shows that the three possibilities illustrated in Figure 2 can all occur. The final step of the stability analysis is to conclude the nonlinear orbital stability of the pulse from its spectral stability. In $\$ 2$, we give an overview of two different strategies, due respectively to [CMPS98] and BGHR03, that allow us to relate spectral and nonlinear stability.

The remainder of this paper is organized as follows. In $\oint_{2}$, we formulate a set of spectral conditions that guarantees the nonlinear stability of travelling wave solutions to a general class of lattice systems. We proceed in $\$ 3$ with an overview of the existence and stability properties of the front and back solutions $q_{f}$ and $q_{b}$. In \$4, we recall the construction of the pulses $(\bar{u}, \bar{w})(\epsilon)$ from [HS10] as this information will be crucial for the stability analysis. Finally, we outline our approach for proving the spectral stability of these pulses in 95 , leaving the technical details to $\$ 6$,

\section{Nonlinear stability}

In this section we formulate a set of spectral conditions that guarantee the nonlinear stability of travelling wave solutions to the lattice system 3

$$
\dot{U}_{j}=\mathcal{F}(U)_{j}=F\left(U_{j-1}, U_{j}, U_{j+1}\right), \quad j \in \mathbb{Z}, \quad U_{j} \in \mathbb{R}^{n}
$$

in which we take $F: \mathbb{R}^{3 n} \rightarrow \mathbb{R}^{n}$ to be $C^{2}$-smooth with $n \geq 1$. We are interested in nontrivial waves that move to the left 4 as described in the following condition.

Hypothesis (HV). The LDE (2.1) admits a solution $U(t)=\bar{U}(t)$ that has

$$
\bar{U}_{j}(t)=\bar{V}(j+c t)
$$

\footnotetext{
${ }^{3}$ Our restriction to nearest-neighbour interactions is arbitrary; any finite-range interaction can be treated in the same fashion.

${ }^{4}$ Waves that move to the right can be treated by switching $j \mapsto-j$.
} 
for some wave profile $\bar{V} \in C^{1}\left(\mathbb{R}, \mathbb{R}^{n}\right)$ and some wave speed $c>0$. The profile $\bar{V}$ satisfies the limits $\bar{V}(\xi) \rightarrow \bar{V}_{ \pm}$as $\xi \rightarrow \pm \infty$ and $\bar{V}^{\prime}$ does not vanish everywhere.

There are two possible approaches to analyzing spectral and nonlinear stability of the travelling wave solution $\bar{U}(t)$. First, $\bar{U}(t)$ can be viewed as a relative periodic orbit of the lattice system (2.1). Indeed, we have

$$
\bar{U}_{j}(t)=\bar{U}_{j-1}(t+1 / c)
$$

for all $t$ so that the profile at times $t=k / c$ for integers $k$ looks like the profile at $t=0$ but shifted by $k$ lattice points to the left. We could therefore use Floquet theory applied to the infinite-dimensional ordinary differential equation (2.1) to identify appropriate spectral stability assumptions that imply nonlinear stability. This program was carried out in CMPS98, and we will review it in 2.1 . The second approach focuses on the profile $\bar{V}(\xi)$ : this function satisfies a functional differential equation of mixed type, and we might expect that the linearization of this MFDE about the profile $\bar{V}$ gives an appropriate notion of spectral stability that one could utilize to show nonlinear stability via the associated Green's functions. This approach was first used in BGHR03, and we will also follow it in this paper. Our choice is motivated primarily by the fact that our existence proof for the pulses also relies on a detailed analysis of an MFDE, which will allow us to reuse many of the tools developed in HS10. We now describe this framework in more detail.

We consider solutions $U(t)$ to (2.1) that take values in the sequence space

$$
\ell^{\infty}=\left\{U \in\left(\mathbb{R}^{n}\right)^{\mathbb{Z}}:|U|_{\ell \infty}:=\sup _{j \in \mathbb{Z}}\left|U_{j}\right|<\infty\right\} .
$$

In addition, we introduce the spaces

$$
\ell^{p}=\left\{U \in\left(\mathbb{R}^{n}\right)^{\mathbb{Z}}:|U|_{\ell^{p}}:=\left[\sum_{j \in \mathbb{Z}}\left|U_{j}\right|^{p}\right]^{1 / p}<\infty\right\}
$$

for $1 \leq p<\infty$, which we use to characterize perturbations from the wave $\bar{U}$.

The discreteness of the spatial variable $j$ implies that one cannot pass to a reference frame in which the wave $\bar{U}$ becomes stationary without destroying the structure of (2.1). This is the central difficulty that needs to be addressed in any stability analysis concerning $\bar{U}$. We will handle this problem using the approach pioneered by Benzoni-Gavage and coworkers BG02, BGHR03. The main idea is to fill the space between the lattice points by looking for solutions to (2.1) that can be written as $U_{j}(t)=V(t, j+c t)$ for some $V \in C\left(\mathbb{R}_{+} \times \mathbb{R}, \mathbb{R}^{n}\right)$. This function must hence solve the nonlocal PDE

$$
\partial_{t} V(t, \xi)+c \partial_{\xi} V(t, \xi)=F(V(t, \xi-1), V(t, \xi), V(t, \xi+1)) .
$$

By construction, $V(t, \xi)=\bar{V}(\xi)$ is now a stationary solution to this problem and it is therefore natural to proceed by linearizing (2.6) around $\bar{V}$.

The main result of this section, Proposition 2.1. shows that stability properties for $\bar{U}$ can indeed be read off from spectral properties of the operator $\mathcal{L}$ : $W^{1, \infty}\left(\mathbb{R}, \mathbb{C}^{n}\right) \rightarrow L^{\infty}\left(\mathbb{R}, \mathbb{C}^{n}\right)$ that is given by

$$
\mathcal{L} V=-c V^{\prime}+L V .
$$

Here we have introduced the notation

$$
[L V](\xi)=\sum_{j=-1}^{1} A_{j}(\xi) V(\xi+j),
$$




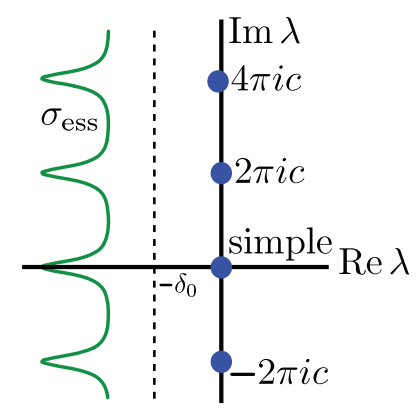

Figure 3. Illustration of the spectral conditions (S1)-(S3). The spectrum of $\mathcal{L}$ is invariant under the operation $\lambda \mapsto \lambda+2 \pi i c$.

in which $A_{-1}(\xi)=D_{1} F(\bar{V}(\xi-1), \bar{V}(\xi), \bar{V}(\xi+1))$, and $A_{0}(\xi)$ and $A_{1}(\xi)$ are defined analogously.

An easy computation shows that

$$
e_{-2 \pi i k}(\mathcal{L}-\lambda) e_{2 \pi i k}=\mathcal{L}-2 \pi i k c-\lambda
$$

for all $k \in \mathbb{Z}$, in which the exponential shift operator $e_{\nu}$ is defined by

$$
\left[e_{\nu} V\right](\xi)=e^{\nu \xi} V(\xi) \text {. }
$$

In particular, the spectrum of $\mathcal{L}$ is invariant under the operation $\lambda \mapsto \lambda+2 \pi i c$. This should be seen as a direct consequence of the fact that the lattice cannot see modulations on a scale finer than the lattice size. Since we have $\mathcal{L} \bar{V}^{\prime}=0$, the entire set $2 \pi i c \mathbb{Z}$ is contained in the spectrum of $\mathcal{L}$.

Our stability result imposes the conditions (S1)-(S3) listed below. As illustrated in Figure 3, these conditions basically state that the spectrum of $\mathcal{L}$ is contained in the open left half-plane, except for isolated simple eigenvalues at $\lambda \in 2 \pi i c \mathbb{Z}$ that arise as a consequence of translational invariance.

Hypothesis (S1). There exists $\delta_{0}>0$ such that $\mathcal{L}-\lambda$ is invertible as a map from $W^{1, \infty}\left(\mathbb{R}, \mathbb{C}^{n}\right)$ into $L^{\infty}\left(\mathbb{R}, \mathbb{C}^{n}\right)$ for all $\lambda \in \mathbb{C} \backslash 2 \pi i c \mathbb{Z}$ that have $\operatorname{Re} \lambda \geq-\delta_{0}$.

Hypothesis (S2). The only nontrivial solutions $V \in W^{1, \infty}\left(\mathbb{R}, \mathbb{C}^{n}\right)$ of $\mathcal{L} V=0$ are $V=\bar{V}^{\prime}$ and scalar multiples thereof.

Hypothesis (S3). The equation $\mathcal{L} V=\bar{V}^{\prime}$ has no solutions $V \in W^{1, \infty}\left(\mathbb{R}, \mathbb{C}^{n}\right)$.

The following proposition states that the preceding spectral stability properties imply nonlinear stability of the travelling wave with respect to the original lattice dynamical system.

Proposition 2.1. Consider the LDE (2.1) and suppose that (HV) and (S1)-(S3) are satisfied. Then there exist constants $\delta>0, C>0$ and $\beta>0$ such that the following holds true for any $1 \leq p \leq \infty$. For any initial condition $U^{0} \in \ell^{\infty}$ that can be bounded by

$$
\left|U^{0}-\bar{U}(0)\right|_{\ell^{p}} \leq \delta
$$

there is a $\vartheta_{*} \in \mathbb{R}$ such that the solution $x$ of (1.1) with $U(0)=U^{0}$ satisfies

$$
\left|U(t)-\bar{U}\left(t+\vartheta_{*}\right)\right|_{\ell^{p}} \leq C e^{-\beta t}\left|U^{0}-\bar{U}(0)\right|_{\ell^{p}}
$$

for every $t>0$. 
We prove this result in 2.2 , exploiting an identity derived in BGHR03, that describes the linearized flow of (2.1) near the wave $\bar{U}$ in terms of Green's functions for $\mathcal{L}-\lambda$. We show that these Green's functions are meromorphic with simple poles at $\lambda \in 2 \pi i c \mathbb{Z}$, allowing us to properly isolate the nondecaying part of the linearized flow that is responsible for the asymptotic phase shift.

We remark that a similar nonlinear stability analysis was performed in BHSZ10 for the far more difficult setting where the translational eigenvalues at $\lambda \in 2 \pi i c \mathbb{Z}$ are embedded in the essential spectrum. This situation is encountered when studying Lax shocks in semi-discrete conservation laws. To analyze the corresponding Green's functions for $\mathcal{L}-\lambda$, the system $\mathcal{L} V=0$ was rewritten as an ODE posed on the augmented space $L^{2}\left([-1,1], \mathbb{C}^{n}\right) \times \mathbb{C}^{n}$. The resulting enlarged system can be shown to admit exponential dichotomies, which can in turn be used to define the desired Green's functions and analyze their behaviour. Our approach here uses a more direct method, which does not require us to work in this augmented state space and hence avoids many technical issues related to the regularity of solutions.

In the special case that $p=\infty$ and $\bar{U}$ is a pulse, i.e., $\bar{V}_{-}=\bar{V}_{+}$in (HV), the conclusions in Proposition 2.1 can be derived by combining the results in BGHR03. and [CMPS98. In the latter paper, the authors avoid the nonlocal comoving PDE (2.6) and study (2.1) directly. They exploit the fact that the wave $\bar{U}$ is shift-periodic in the sense of (2.3). By developing a shift-periodic version of Floquet theory, the authors are able to analyze the stability properties of the discrete Nagumo equation (1.12). The main disadvantage of this approach compared to the use of Green's function techniques is that spectral conditions that are weaker than (S1)(S3) cannot be readily accommodated. In 2.1 we discuss these issues in greater detail.

2.1. Shift-periodic orbits. In this part, we discuss the results obtained in CMPS98, where a shift-periodic version of Floquet theory was developed to analyze the stability of a travelling wave solution $\bar{U}$ to (2.1). In particular, we will show how Proposition 2.1 with $p=\infty$ and $\bar{V}_{-}=\bar{V}_{+}$can be derived from these results by relating the spectrum of the appropriate shift-periodic monodromy operator to the spectrum of the operator $\mathcal{L}$ defined in (2.7).

Looking for solutions to (2.1) of the form $U(t)=\bar{U}(t)+W(t)$, we find that $W$ must satisfy the time-dependent LDE

$$
\dot{W}(t)=D \mathcal{F}(\bar{U}(t)) W(t)+\mathcal{N}(t, W(t)),
$$

in which

$$
\mathcal{N}(t, W)=\mathcal{F}(\bar{U}(t)+W)-\mathcal{F}(\bar{U}(t))-D \mathcal{F}(\bar{U}(t)) W .
$$

Since $\bar{V}$ is bounded, we have $\mathcal{N}(t, W)=O\left(|W|^{2}\right)$ and $D_{2} \mathcal{N}(t, W)=O(|W|)$ as $W \rightarrow 0$, uniformly for $t \in \mathbb{R}$. Here $D_{2}$ denotes differentiation with respect to the second variable.

Upon introducing the right-shift operator $\mathcal{S} \in \mathcal{L}\left(\ell^{\infty}\right)$ that acts as $(\mathcal{S} U)_{j}=U_{j-1}$, the shift-periodicity of the wave $\bar{U}$ can be formulated as

$$
\bar{U}(t)=\mathcal{S} \bar{U}\left(t+c^{-1}\right), \quad t \in \mathbb{R} .
$$

Since $\mathcal{F}$ commutes with shifts on the lattice, the nonautonomous terms in (2.13) are also shift-periodic in the sense that

$$
D \mathcal{F}(\bar{U}(t)) W=\mathcal{S} D \mathcal{F}\left(\bar{U}\left(t+c^{-1}\right)\right) \mathcal{S}^{-1} W, \quad \mathcal{N}(t, W)=\mathcal{S} \mathcal{N}\left(t+c^{-1}, \mathcal{S}^{-1} W\right) .
$$


These observations show that it indeed makes sense to develop a shift-periodic version of Floquet theory to study the stability properties of $\bar{U}$. As a first step, one needs to identify the appropriate monodromy operator associated to the linear part of (2.13). Using standard ODE techniques, one can see that for any $t_{0} \in \mathbb{R}$ and $j_{0} \in \mathbb{Z}$, the LDE

$$
\dot{W}(t)=D \mathcal{F}(\bar{U}(t)) W(t)
$$

with the initial condition $W_{j}\left(t_{0}\right)=\delta_{j j_{0}}$ has a unique solution $W=W^{t_{0} j_{0}}$ that is defined for all $t \in \mathbb{R}$. This allows us to define a Green's function $\mathcal{G}\left(t, t_{0}\right) \in \mathcal{L}\left(\ell^{\infty}\right)$ by writing

$$
\mathcal{G}_{j j_{0}}\left(t, t_{0}\right)=W_{j}^{t_{0} j_{0}}(t)
$$

and observing that the unique solution to (2.17) with the initial condition $W\left(t_{0}\right)=z$ is given by

$$
W_{j}(t)=\left(\mathcal{G}\left(t, t_{0}\right) z\right)_{j}:=\sum_{j_{0} \in \mathbb{Z}} \mathcal{G}_{j j_{0}}\left(t, t_{0}\right) z_{j_{0}} .
$$

Differentiating (2.1), we see that

$$
\dot{\bar{U}}(t)=\mathcal{G}(t, 0) \dot{\bar{U}}(0)
$$

holds for all $t \in \mathbb{R}$ and hence, exploiting (2.15),

$$
\dot{\bar{U}}(0)=\mathcal{S G}\left(c^{-1}, 0\right) \dot{\bar{U}}(0) .
$$

Writing $\mathcal{R}=\mathcal{S G}\left(c^{-1}, 0\right)$, we hence expect $\mathcal{R}$ to be the appropriate generalization of the monodromy map. As usual, we define the spectrum of $\mathcal{R}$ to be the set

$$
\sigma(\mathcal{R})=\left\{\zeta \in \mathbb{C} \mid \mathcal{R}-\zeta: \ell^{\infty} \rightarrow \ell^{\infty} \text { is not invertible }\right\}
$$

The following result shows that $\mathcal{R}$ can indeed be used to study the stability of $\bar{U}$.

Proposition 2.2 (See [CMPS98, Thm. A] and [CMPS98, §5]). Consider the LDE (2.1) and suppose that $(H V)$ is satisfied 5 Suppose furthermore that

$$
\sigma(\mathcal{R}) \backslash\{1\} \subset\{\zeta:|\zeta|<1\}
$$

and that the eigenvalue $1 \in \sigma(\mathcal{R})$ is simple. Then the conclusions of Proposition 2.1 hold for $p=\infty$, i.e., $\bar{U}$ is asymptotically stable in $\ell^{\infty}$ with an asymptotic phase shift.

This result was established in CMPS98 by constructing a local $\ell^{\infty}$-coordinate system around the wave $\bar{U}$ and analyzing the monodromy map in these new coordinates. The arguments used to construct this coordinate system are very technical and it is therefore not clear if they work in $\ell^{p}$ with $1 \leq p<\infty$. In addition, it is not at all obvious how spectral conditions that are weaker than (2.23) can be treated.

In practice, it is often hard to analyze the operator $\mathcal{R}$ directly. Fortunately, one can use a spectral mapping result due to Benzoni-Gavage and coworkers BGHR03. to work around this difficulty and analyze the operator $\mathcal{L}$ from (2.7) instead. As a

\footnotetext{
${ }^{5}$ The assumptions in CMPS98 concerning the nonlinearity $\mathcal{F}$ and the travelling wave $\bar{U}$ are weaker than those stated here.
} 
preparation, we introduce the point spectra and essential spectra of the operators $\mathcal{R}$ and $\mathcal{L}$ that are defined by

$(2.24)$

$$
\begin{aligned}
& \sigma_{\text {ess }}(\mathcal{L})=\{\lambda \in \mathbb{C} \mid \mathcal{L}-\lambda \text { is not a Fredholm operator with index zero }\}, \\
& \sigma_{p}(\mathcal{L})=\left\{\lambda \in \mathbb{C} \backslash \sigma_{\text {ess }}(\mathcal{L}) \mid \mathcal{L} V=\lambda V \text { for some nonzero } V \in W^{1, \infty}\left(\mathbb{R}, \mathbb{C}^{n}\right)\right\}, \\
& \sigma_{\text {ess }}(\mathcal{R})=\{\zeta \in \mathbb{C} \mid \mathcal{R}-\zeta \text { is not a Fredholm operator with index zero }\}, \\
& \sigma_{p}(\mathcal{R})=\left\{\zeta \in \mathbb{C} \backslash \sigma_{\text {ess }}(\mathcal{R}) \mid \mathcal{R} W=\lambda W \text { for some nonzero } W \in \ell^{\infty}\right\} .
\end{aligned}
$$

Lemma 2.3 (See BGHR03, §3]). Consider the LDE (2.1) and suppose that (HV) is satisfied. Suppose furthermore that $\bar{V}(\xi)-\bar{V}_{ \pm}=O\left(e^{-\beta|\xi|}\right)$ as $\xi \rightarrow \pm \infty$ for some $\beta>0$. Then we have the spectral mapping

$$
\exp \left[c^{-1} \sigma_{p}(\mathcal{L})\right]=\sigma_{p}(\mathcal{R}) \backslash\{0\} .
$$

In addition, if $\zeta$ is a nonzero eigenvalue of $\mathcal{R}$ and $\zeta=e^{\lambda / c}$, then the multiplicity of $\lambda$ as an eigenvalue of $\mathcal{L}$ equals the multiplicity of $\zeta$ as an eigenvalue of $\mathcal{R}$. Finally, in the special case $\bar{V}_{-}=\bar{V}_{+}$, we also have the spectral mapping

$$
\exp \left[c^{-1} \sigma_{\text {ess }}(\mathcal{L})\right]=\sigma_{\text {ess }}(\mathcal{R}) \backslash\{0\} .
$$

Proof. The statements concerning the point spectrum follow from BGHR03, Thm. 3.8]. In the special case $\bar{V}_{-}=\bar{V}_{+}$, the arguments in the proof of [BGHR03, Lem. 3.4] can be used to strengthen the inclusions [BGHR03, Eq. (3.13)] and obtain (2.26).

This spectral mapping result provides a link between the spectral conditions (S1)(S3) concerning $\mathcal{L}$ and the spectral conditions on $\mathcal{R}$ that appear in Proposition 2.2 .

Corollary 2.4. Consider the LDE (2.1) and suppose that (HV) and (S1)-(S3) are satisfied. Suppose furthermore that $\bar{V}_{-}=\bar{V}_{+}$. Then the conclusions of Proposition 2.1 hold for $p=\infty$, i.e., $\bar{U}$ is asymptotically stable in $\ell^{\infty}$ with an asymptotic phase shift.

Proof. Condition (S1) implies that $0 \in \sigma(\mathcal{L})$ is isolated, which in view of MP99a, Thm. A] means that the characteristic equations

$$
c z-\sum_{j=-1}^{1}\left[\lim _{\xi \rightarrow \pm \infty} A_{j}(\xi)\right] e^{z j}=0
$$

cannot have roots with $z \in i \mathbb{R}$. By applying MP99a, Prop 5.3] to MP99b, Eq. (6.4)], we hence find that $\bar{V}$ approaches its limits $\bar{V}_{ \pm}$at an exponential rate. We may hence use Lemma 2.3 to verify the spectral conditions on $\mathcal{R}$ mentioned in Proposition 2.2.

2.2. Proof of Proposition 2.1, Throughout the remainder of this section we give a direct proof of Proposition 2.1, bypassing the monodromy operator $\mathcal{R}$ and the local coordinate system around $\bar{U}$ developed in CMPS98. The key ingredient that we will use is that the Green's function $\mathcal{G}$ for the lattice system (2.17) can be related to the Green's functions $G\left(\xi, \xi_{0}, \lambda\right)$ that solve

$$
(\mathcal{L}-\lambda) G\left(\cdot, \xi_{0}, \lambda\right)=\delta\left(\cdot-\xi_{0}\right)
$$

in the sense of distributions. Indeed, we have the following representation for $\mathcal{G}$. 


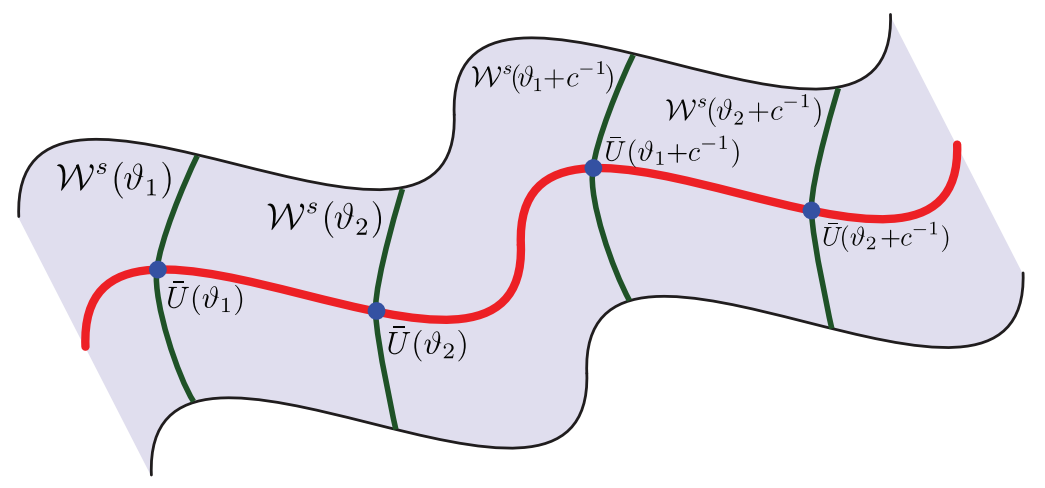

Figure 4. The entire orbital neighbourhood of the wave $\bar{U}$ is spanned by the branches $\mathcal{W}^{s}(\vartheta)$.

Lemma 2.5 ([BGHR03, Thm. 4.2]). Consider the LDE (2.1) and suppose that $(H V)$ is satisfied. For each fixed sufficiently large $\gamma \gg 1$, each pair $t>t_{0}$ and all $j, j_{0} \in \mathbb{Z}$, we have

$$
\mathcal{G}_{j j_{0}}\left(t, t_{0}\right)=-\frac{1}{2 \pi i} \int_{\gamma-i \pi c}^{\gamma+i \pi c} e^{\lambda\left(t-t_{0}\right)} G\left(j+c t, j_{0}+c t_{0}, \lambda\right) d \lambda .
$$

In view of the symmetry (2.9), we see that

$$
G\left(\xi, \xi_{0}, \lambda+2 \pi i k c\right)=e^{2 \pi i k\left(\xi_{0}-\xi\right)} G\left(\xi, \xi_{0}, \lambda\right)
$$

for all $k \in \mathbb{Z}$, which implies that the integration contour in (2.29) can be shifted to the left as long as $G\left(\xi, \xi_{0}, \cdot\right)$ remains analytic. In fact, we will show that $G\left(\xi, \xi_{0}, \lambda\right)$ is meromorphic for $\lambda \approx 0$. This will allow us to shift the integration contour to the left of the imaginary axis and write

$$
\mathcal{G}\left(t, t_{0}\right)=\mathcal{E}\left(t, t_{0}\right)+\widetilde{\mathcal{G}}\left(t, t_{0}\right)
$$

in which $\mathcal{G}$ decays exponentially and $\mathcal{E}\left(t, t_{0}\right)$ projects onto the center part of the flow induced by $\mathcal{G}$, which is spanned by $\dot{\bar{U}}(t)$. For every $\vartheta \in \mathbb{R}$, we will construct a codimension one branch of initial conditions $\mathcal{W}^{s}(\vartheta)$ that is transverse to $\dot{\bar{U}}(\vartheta)$ at $\bar{U}(\vartheta)$, for which the associated solutions to the lattice system (2.1) converge exponentially to $\bar{U}(\cdot+\vartheta)$. As illustrated in Figure 4 , the entire orbital neighbourhood of the wave $\bar{U}$ can subsequently be spanned by such initial conditions by varying $\vartheta \in \mathbb{R}$.

We start our analysis by constructing the Green's functions $G\left(\xi, \xi_{0}, \lambda\right)$ associated to the operators $\mathcal{L}-\lambda$. As a preparation, let us introduce the limiting operator

$$
\mathcal{L}_{\infty} V=-c V^{\prime}+L_{\infty} V
$$

in which

$$
\left[L_{\infty} V\right](\xi)=\sum_{j=-1}^{1}\left[\lim _{\xi \rightarrow \infty} A_{j}(\xi)\right] V(\xi+j) .
$$

Arguing as in the proof of Corollary 2.4 we may use [MP99a, Thm. 4.1] to conclude that $\mathcal{L}_{\infty}$ is invertible and has a Green's function $G_{\infty}(\xi)$ that is continuous on $\mathbb{R} \backslash\{0\}$, decays exponentially as $\xi \rightarrow \pm \infty$ and satisfies $\mathcal{L}_{\infty} G_{\infty}=\delta(\cdot)$ in the sense 
of distributions. This function $G_{\infty}$ can be used to construct Green's functions for $\mathcal{L}-\lambda$.

Lemma 2.6. Suppose that (S1) holds. Consider any $\lambda \in \mathbb{C} \backslash \sigma(\mathcal{L})$. Then the function

$$
G\left(\xi, \xi_{0}, \lambda\right)=G_{\infty}\left(\xi-\xi_{0}\right)-\left[(\mathcal{L}-\lambda)^{-1}\left[L-L_{\infty}-\lambda\right] G_{\infty}\left(\cdot-\xi_{0}\right)\right](\xi)
$$

satisfies $(\mathcal{L}-\lambda) G\left(\cdot, \xi_{0}, \lambda\right)=\delta\left(\cdot-\xi_{0}\right)$ in the sense of distributions. In addition, $G\left(\cdot, \xi_{0}, \lambda\right)$ is $C^{0}$-smooth on $\mathbb{R} \backslash\left\{\xi_{0}\right\}$ and $C^{1}$-smooth on $\mathbb{R} \backslash\left\{\xi_{0}-1, \xi_{0}, \xi_{0}+1\right\}$. Finally, there exist $\beta>0$ and $C>0$ such that

$$
\left|G\left(\xi, \xi_{0}, \lambda\right)\right| \leq C e^{-\beta\left|\xi-\xi_{0}\right|}
$$

holds for all $\xi, \xi_{0} \in \mathbb{R}$.

Proof. The bound (2.35) can be computed directly from (2.34) by using the exponential decay of $G_{\infty}$ and evaluating $(\mathcal{L}-\lambda)^{-1}$ on the space $L_{\beta}^{\infty}$ if $\xi<\xi_{0}$ and $L_{-\beta}^{\infty}$ if $\xi>\xi_{0}$. Here we have used the shorthand $L_{\beta}^{\infty}=e_{\beta}\left(L^{\infty}\left(\mathbb{R}, \mathbb{C}^{n}\right)\right)$ with norm $\|f\|_{L_{\beta}^{\infty}}=\left\|e_{-\beta} f\right\|_{L^{\infty}}$, where $e_{ \pm \beta}$ are as in (2.10). The remaining properties can be verified by a direct calculation.

We will write $\mathcal{L}^{*}: W^{1, \infty}\left(\mathbb{R}, \mathbb{C}^{n}\right) \rightarrow L^{\infty}\left(\mathbb{R}, \mathbb{C}^{n}\right)$ for the formal adjoint of $\mathcal{L}$, which is given by

$$
\left[\mathcal{L}^{*} V\right](\xi)=c V^{\prime}(\xi)+\sum_{j=-1}^{1} A_{j}^{*}(\xi-j) V(\xi-j) .
$$

We remark that (S1) and (S2) in combination with [MP99a, Thm. A] imply that $\operatorname{dim} \operatorname{Ker} \mathcal{L}^{*}=1$. In particular, we have

$$
\operatorname{Ker} \mathcal{L}^{*}=\operatorname{span}\{d\}
$$

for some nonzero $d \in C^{1}(\mathbb{R}, \mathbb{R})$ that we will use throughout the remainder of this section. The following result describes the pole that arises as the contour of integration in (2.29) is shifted to the left of the imaginary axis.

Lemma 2.7. Consider the LDE (2.1) and suppose that (HV) and (S1)-(S3) are satisfied. Then there exists $\delta_{\lambda}>0$ such that for all $\lambda \in \mathbb{C}$ with $0<|\lambda|<\delta_{\lambda}$ we have the representation

$$
G\left(\xi, \xi_{0}, \lambda\right)=E\left(\xi, \xi_{0}, \lambda\right)+\widetilde{G}\left(\xi, \xi_{0}, \lambda\right) .
$$

Here the meromorphic term can be written as

$$
E\left(\xi, \xi_{0}, \lambda\right)=-\left[\lambda \int_{-\infty}^{\infty} d\left(\xi^{\prime}\right)^{*} \bar{V}^{\prime}\left(\xi^{\prime}\right) d \xi^{\prime}\right]^{-1} \bar{V}^{\prime}(\xi) d\left(\xi_{0}\right)^{*}
$$

while the remainder $\widetilde{G}\left(\xi, \xi_{0}, \lambda\right)$ depends analytically on $\lambda$ in the region $|\lambda|<\delta_{\lambda}$ and satisfies the bound

$$
\left|\widetilde{G}\left(\xi, \xi_{0}, \lambda\right)\right| \leq C e^{-\beta\left|\xi-\xi_{0}\right|}
$$

for some $C>0$ and $\beta>0$. 
Proof. Without loss of generality, we will assume that $d$ does not vanish identically on either $\mathbb{R}_{-}$or $\mathbb{R}_{+}$. Indeed, if this condition fails, the proof given here can be modified by choosing different half-lines. Arguing as in [HVL09, §3] 6 we may show that the inhomogeneous equations $\mathcal{L} V=f$ can be solved on the half-lines $\mathbb{R}_{ \pm}$. Proceeding as in [HVL09, $\S 6]$, we can show that for any $f \in L^{\infty}\left(\mathbb{R}, \mathbb{C}^{n}\right)$, a bounded pair $\left(V^{-}, V^{+}\right)=: L_{*} f$ with $V^{-} \in C\left((-\infty, 1], \mathbb{C}^{n}\right)$ and $V^{+} \in C\left([-1, \infty), \mathbb{C}^{n}\right)$ can be constructed in such a way that $\mathcal{L} V^{ \pm}=f$ on $\mathbb{R}_{ \pm}$. In addition, $V^{+}$and $V^{-}$agree on $[-1,1]$ if and only if the integral

$$
M(f)=\int_{-\infty}^{\infty} d(\xi)^{*} f(\xi) d \xi
$$

vanishes 7 Now, for $\lambda \neq 0$, notice that $(\mathcal{L}-\lambda)^{-1} f$ must be given by

$$
\left(V^{-}, V^{+}\right)=L_{*} f+\lambda L_{*}\left(V^{-}, V^{+}\right)+\kappa \bar{V}^{\prime}
$$

for some $\kappa \in \mathbb{C}$, which in view of the requirement $M\left(f+\lambda\left(V^{-}, V^{+}\right)\right)=0$ must satisfy

$$
\kappa M\left(\bar{V}^{\prime}\right)=-\frac{1}{\lambda} M(f)-M\left(L_{*} f\right)-\lambda M\left(L_{*}\left(V^{+}, V^{-}\right)\right) .
$$

Observing that $M\left(\bar{V}^{\prime}\right) \neq 0$ in view of (S3) and MP99a, Thm. A] allows us to write

$$
(\mathcal{L}-\lambda)^{-1} f=-\frac{M(f)}{\lambda M\left(\bar{V}^{\prime}\right)} \bar{V}^{\prime}+\mathcal{B}(\lambda) f
$$

in which $\|\mathcal{B}(\lambda)\| \leq C$ for all $|\lambda| \leq \delta_{\lambda}$. The proof can be completed by computing

$$
\begin{aligned}
\int_{-\infty}^{\infty} d(\xi)^{*}\left[\left(L-L_{\infty}\right) G_{\infty}\left(\cdot-\xi_{0}\right)\right](\xi) d \xi & =\int_{-\infty}^{\infty} d(\xi)^{*}\left[\left(\mathcal{L}-\mathcal{L}_{\infty}\right) G_{\infty}\left(\cdot-\xi_{0}\right)\right](\xi) d \xi \\
& =-\int_{-\infty}^{\infty} d(\xi)^{*}\left[\mathcal{L}_{\infty} G_{\infty}\left(\cdot-\xi_{0}\right)\right](\xi) d \xi \\
& =-d\left(\xi_{0}\right)^{*}
\end{aligned}
$$

and using weighted norms as in the proof of Lemma 2.6 to estimate the remainder $\widetilde{G}\left(\xi, \xi_{0}, \lambda\right)$.

Using the symmetry (2.30), we can now shift the integration path in (2.29) slightly to the left of the imaginary axis. We pick up a residue from the simple pole at $\lambda=0$ that comes from the meromorphic term $E\left(\xi, \xi_{0}, \lambda\right)$.

Corollary 2.8. Consider the LDE (2.1) and suppose that (HV) and (S1)-(S3) are satisfied. For any pair $t>t_{0}$ and any $j, j_{0} \in \mathbb{Z}$, we have the representation

$$
\mathcal{G}_{j j_{0}}\left(t, t_{0}\right)=\mathcal{E}_{j j_{0}}\left(t, t_{0}\right)+\widetilde{\mathcal{G}}_{j j_{0}}\left(t, t_{0}\right),
$$

in which

$$
\mathcal{E}_{j, j_{0}}\left(t, t_{0}\right)=\left[\int_{-\infty}^{\infty} d(\xi)^{*} \bar{V}^{\prime}(\xi) d \xi\right]^{-1} \bar{V}^{\prime}(j+c t) d\left(j_{0}+c t_{0}\right)^{*}
$$

\footnotetext{
${ }^{6}$ Condition (HB) in HVL09 may not be true. However, since $\mathcal{L}^{*}$ has a one-dimensional kernel, the integration interval in [HVL09, Eq. (3.28)] can be chosen to be arbitrarily small. In addition, we only need this equation to hold for a single $\xi>0$ and a single $\xi<0$.

${ }^{7}$ We emphasize that we do not need the Hale inner product [HVL09] Eq. (2.7)] to be nondegenerate for the 'if' part of this statement to work. Indeed, we only need [HVL09, Lem. 4.3(ii)] to hold for $\xi=0$, after which we may conclude $\beta(0)=1$ in [MPVL, Eq. (4.12)].
} 
while $\widetilde{\mathcal{G}}$ satisfies the bound

$$
\left|\widetilde{\mathcal{G}}_{j j_{0}}\left(t, t_{0}\right)\right| \leq C e^{-\beta\left(t-t_{0}\right)} e^{-\beta\left|j+c t-j_{0}-c t_{0}\right|}
$$

for some $C>0$ and $\beta>0$.

For any $t \in \mathbb{R}$, we introduce the operator $\Pi^{c}(t) \in \mathcal{L}\left(\ell^{p}\right)$ that acts as

$$
\Pi^{c}(t) W=\mathcal{E}(t, t) W
$$

and write $\Pi^{s}(t)=I-\Pi^{c}(t)$. Although it is not immediately clear from the representation (2.47), the operators $\Pi^{c}(t)$ and hence $\Pi^{s}(t)$ are projections that correspond to the center and stable parts of the flow induced by $\mathcal{G}$.

Lemma 2.9. Consider the LDE (2.1) and suppose that (HV) and (S1)-(S3) are satisfied. Then the operators $\Pi^{c}(t)$ and $\Pi^{s}(t)$ are projections and for any $t>t_{0}$ we have

$$
\mathcal{G}\left(t, t_{0}\right)=\mathcal{E}\left(t, t_{0}\right) \Pi^{c}\left(t_{0}\right)+\widetilde{\mathcal{G}}\left(t, t_{0}\right) \Pi^{s}\left(t_{0}\right) .
$$

Proof. To see that $\left(\Pi^{c}(t)\right)^{2}=\Pi^{c}(t)$, it suffices to show that

$$
\sum_{j \in \mathbb{Z}} d(j+c t)^{*} \bar{V}^{\prime}(j+c t)=\int_{-\infty}^{\infty} \bar{d}(\xi)^{*} \bar{V}^{\prime}(\xi) d \xi
$$

To see this, we note that a simple computation (see e.g. [HVL09, Eq. (4.8)]) yields

$$
c d(\xi)^{*} \bar{V}^{\prime}(\xi)=\sum_{j=-1}^{1} \int_{0}^{j} d(\xi+\theta-j)^{*} A_{j}(\xi+\theta-j) \bar{V}^{\prime}(\xi+\theta) d \theta
$$

for all $\xi \in \mathbb{R}$, which ensures that the sum and integral in (2.51) pick up exactly the same terms.

The explicit form of $\mathcal{E}$ and the computation above show that $\mathcal{E}\left(t, t_{0}\right)=$ $\mathcal{E}\left(t, t_{0}\right) \Pi^{c}\left(t_{0}\right)$, which implies $\mathcal{E}\left(t, t_{0}\right) \Pi^{s}\left(t_{0}\right)=0$. In addition, using (2.20) we find $\mathcal{G}\left(t, t_{0}\right) \Pi^{c}\left(t_{0}\right)=\mathcal{E}\left(t, t_{0}\right)$, which implies $\widetilde{\mathcal{G}}\left(t, t_{0}\right) \Pi^{c}\left(t_{0}\right)=0$ and completes the proof.

Before we turn to the proof of our nonlinear stability result, we need to introduce the family of weighted function spaces

$$
B C_{\eta}(I, Y)=\left\{W \in C(I, Y):\|W\|_{\eta}:=\sup _{\xi \in I} e^{-\eta|\xi|}\|W(\xi)\|<\infty\right\},
$$

in which $\eta \in \mathbb{R}, I \subset \mathbb{R}$ is an interval and $Y$ is a Banach space. We will write $W \in B C_{\eta}^{1}(I, Y)$ if both $W$ and $W^{\prime}$ belong to $B C_{\eta}(I, Y)$.

Proof of Proposition 2.1. Recall the constant $\beta>0$ from Corollary 2.8. Let us consider any $W_{s} \in$ Range $\Pi^{s}(0)$ and consider the fixed point problem

$$
\begin{aligned}
W(t)= & \widetilde{\mathcal{G}}(t, 0) W_{s}+\int_{0}^{t} \widetilde{\mathcal{G}}\left(t, t_{0}\right) \Pi^{s}\left(t_{0}\right) \mathcal{N}\left(t_{0}, W\left(t_{0}\right)\right) d t_{0} \\
& +\int_{\infty}^{t} \mathcal{E}\left(t, t_{0}\right) \Pi^{c}\left(t_{0}\right) \mathcal{N}\left(t_{0}, W\left(t_{0}\right)\right) d t_{0}
\end{aligned}
$$

posed on the space $B C_{-\beta / 2}\left([0, \infty), \ell^{p}\right)$. One may show in a standard fashion that (2.54) admits a unique solution $W_{*}\left(W_{s}\right)$ for every sufficiently small $W_{s}$, with $\Pi^{s}(0) W_{*}\left(W_{s}\right)(0)=W_{s}$ and $\Pi^{c}(0) W_{*}\left(W_{s}\right)(0)=O\left(\left(W_{s}\right)^{2}\right)$. By construction, the function $\bar{U}+W_{*}\left(W_{s}\right)$ now satisfies (2.1). 


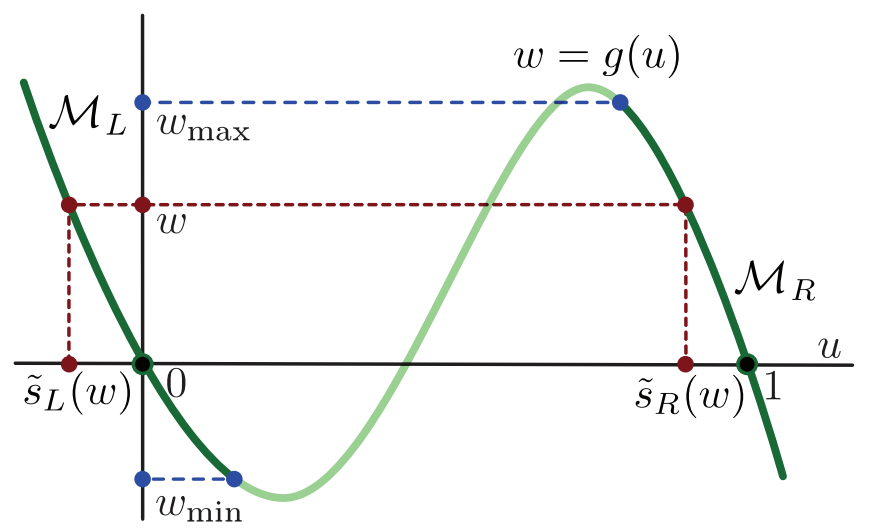

FiguRE 5. Illustrated here are the definitions of $w_{\min }, w_{\max }, \widetilde{s}_{L}$, $\widetilde{s}_{R}, \mathcal{M}_{L}$ and $\mathcal{M}_{R}$.

We have hence found a codimension one branch of initial conditions that converge to $\bar{U}$ at an exponential rate. Of course, we may repeat this procedure for any $\vartheta_{*} \in \mathbb{R}$ to find similar branches that converge to $\bar{U}\left(\cdot+\vartheta_{*}\right)$. Together, these branches span the orbital vicinity of $\bar{U}$, which concludes the proof.

\section{Preliminaries: The discrete Nagumo equation}

In this section we introduce the notation that we use throughout the remainder of this paper and gather some results for the linearization around the travelling wave solutions $q_{f}$ and $q_{b}$ to the discrete Nagumo system (1.12). More precisely, we study the MFDE

$$
c_{*} v^{\prime}=L\left(q_{f}\right) v-\lambda v,
$$

related to the front $q_{f}$, along with the MFDE

$$
c_{*} v^{\prime}=L\left(q_{b}\right) v-\lambda v,
$$

related to the back $q_{b}$. Here we have introduced the following notation for any $u \in C(\mathbb{R}, \mathbb{R}):$

$$
[L(u) v](\xi)=\alpha[v(\xi-1)+v(\xi+1)-2 v(\xi)]+g^{\prime}(u(\xi)) v(\xi) .
$$

As a preparation, let us pick closed intervals $I_{L}$ and $I_{R}$ with $0 \in I_{L}$ and $1 \in I_{R}$ that avoid the knees of the cubic, i.e., $g^{\prime}(u)<0$ for all $u \in I_{L} \cup I_{R}$. In addition, let us pick two constants $w_{\min }<0$ and $w_{\max }>0$ in such a way that both $w_{\min }, w_{\max } \in$ $g\left(I_{L}\right) \cap g\left(I_{R}\right)$. We may now define two smooth functions

$$
\widetilde{s}_{L}:\left[w_{\min }, w_{\max }\right] \rightarrow I_{L} \quad \text { and } \quad \widetilde{s}_{R}:\left[w_{\min }, w_{\max }\right] \rightarrow I_{R}
$$

in such a way that

$$
g\left(\widetilde{s}_{L}(w)\right)=g\left(\widetilde{s}_{R}(w)\right)=w \quad \text { for all } w \in\left[w_{\min }, w_{\max }\right] .
$$

In terms of these functions, the manifolds $\mathcal{M}_{L}$ and $\mathcal{M}_{R}$ mentioned in the introduction and depicted in Figure 5 can be written as (3.6)

$$
\mathcal{M}_{L}=\left\{\left(\widetilde{s}_{L}(w), w\right)\right\}_{w \in\left[w_{\min }, w_{\max }\right]} \quad \text { and } \quad \mathcal{M}_{R}=\left\{\left(\widetilde{s}_{R}(w), w\right)\right\}_{w \in\left[w_{\min }, w_{\max }\right]} .
$$


Our first step is to study the autonomous linear equations that arise by taking the limits $\xi \rightarrow \pm \infty$ in (3.1) and (3.2). In fact, we will study the general class of autonomous equations

$$
c(\epsilon) v^{\prime}=L\left(\widetilde{s}_{\#}(\vartheta)\right) v-\lambda v, \quad \text { for } \#=L, R,
$$

in which we take $\epsilon>0$ to be small and allow any $\vartheta \in\left[w_{\min }, w_{\max }\right]$. Looking for solutions of the form $v(\xi)=\mathrm{e}^{z \xi}$, we must solve the characteristic equation

$$
\Delta_{\#}(\epsilon, \vartheta, \lambda, z)=0 \text {, }
$$

in which we have introduced the characteristic functions

$$
\Delta_{\#}(\epsilon, \vartheta, \lambda, z)=c(\epsilon) z-\alpha\left(e^{z}+e^{-z}-2\right)-g^{\prime}\left(\widetilde{s}_{\#}(\vartheta)\right)+\lambda, \quad \text { for } \#=L, R .
$$

Our first result ensures that we can find a vertical strip $-\eta_{*} \leq \operatorname{Re} z \leq \eta_{*}$ for which the characteristic equation (3.8) is free of roots, uniformly for the parameters $(\epsilon, \vartheta, \lambda)$ in which we will be interested. We will use this constant $\eta_{*}$ ubiquitously throughout the rest of this paper.

Lemma 3.1. There exist constants $\delta_{0}>0, \delta_{\epsilon}>0, \eta_{*}>0$ and $\kappa>0$ such that the inequality

$$
\operatorname{Re} \Delta_{\#}(\epsilon, \vartheta, \lambda, z) \geq \kappa>0, \quad \#=L, R,
$$

holds for all $\epsilon \in\left[0, \delta_{\epsilon}\right], \vartheta \in\left[w_{\min }, w_{\max }\right], \lambda \in \mathbb{C}$ with $\operatorname{Re} \lambda \geq-\delta_{0}$ and $z \in \mathbb{C}$ with $|\operatorname{Re} z| \leq \eta_{*}$.

Proof. Pick $\kappa>0$ in such a way that $g^{\prime}\left(\widetilde{s}_{\#}(\vartheta)\right)<-3 \kappa$ for all $\vartheta \in\left[w_{\min }, w_{\max }\right]$, both for $\#=L, R$. Observe that

$$
\operatorname{Re} \Delta_{\#}(\epsilon, \vartheta, \lambda, p+i q)=c(\epsilon) p+\alpha\left(2-\cos q\left(e^{p}+e^{-p}\right)\right)-g^{\prime}\left(\widetilde{s}_{\#}(\vartheta)\right)+\operatorname{Re} \lambda .
$$

Choosing $\eta_{*}$ in such a way that

$$
\left|c(\epsilon) p+\alpha\left(2-e^{p}-e^{-p}\right)\right| \leq \kappa
$$

holds for all $p \in\left[-\eta_{*}, \eta_{*}\right]$, we pick $\delta_{0}=\kappa$ and compute

$\operatorname{Re} \Delta_{\#}(\epsilon, \vartheta, \lambda, p+i q) \geq c(\epsilon) p+\alpha\left(2-e^{p}-e^{-p}\right)-g^{\prime}\left(\widetilde{s}_{\#}(\vartheta)\right)+\operatorname{Re} \lambda \geq-\kappa+3 \kappa-\kappa=\kappa$, as desired.

Returning to the nonautonomous MFDEs (3.1) and (3.2), we introduce the associated operators

$$
\Lambda_{f, \lambda}: B C_{0}^{1}(\mathbb{R}, \mathbb{C}) \rightarrow B C_{0}(\mathbb{R}, \mathbb{C}) \quad \text { and } \quad \Lambda_{b, \lambda}: B C_{0}^{1}(\mathbb{R}, \mathbb{C}) \rightarrow B C_{0}(\mathbb{R}, \mathbb{C})
$$

that act as

$$
\Lambda_{f, \lambda} v=-c_{*} v^{\prime}+L\left(q_{f}\right) v-\lambda v \quad \text { and } \quad \Lambda_{b, \lambda} v=-c_{*} v^{\prime}+L\left(q_{b}\right) v-\lambda v .
$$

In order to describe solutions to the homogeneous system (3.1) that remain bounded on half-lines, we introduce the spaces

$$
\begin{aligned}
\mathcal{P}_{f, \lambda} & =\left\{v \in B C_{0}((-\infty, 1], \mathbb{C}) \mid v \text { solves (3.1) on }(-\infty, 0]\right\}, \\
\mathcal{Q}_{f, \lambda} & =\left\{v \in B C_{0}([-1, \infty), \mathbb{C}) \mid v \text { solves (3.1) on }[0, \infty)\right\},
\end{aligned}
$$

and their counterparts $\mathcal{P}_{b, \lambda}, \mathcal{Q}_{b, \lambda}$ associated to (3.2). 

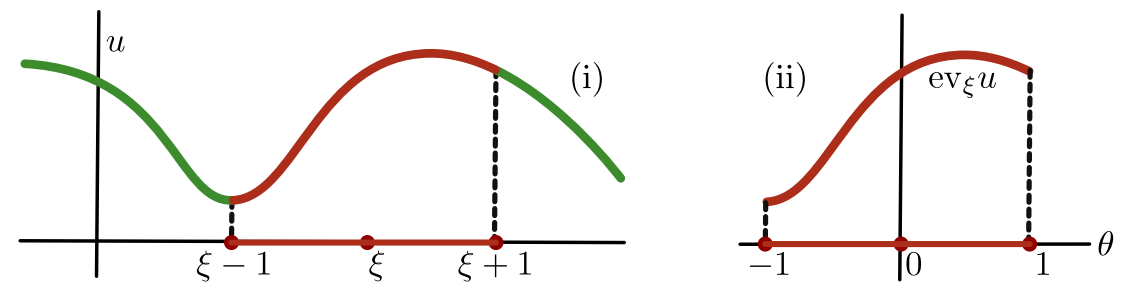

Figure 6. Panel (i) shows the graph of a given function $u$, while panel (ii) illustrates the associated function $\operatorname{ev}_{\xi} u:[-1,1] \rightarrow \mathbb{C}$ for a fixed $\xi$.

In order to capture the initial conditions associated to the functions in these spaces, we will use the notation $\operatorname{ev}_{\xi} u \in C([-1,1], \mathbb{C})$ to denote the state of a function $u \in C(\mathbb{R}, \mathbb{C})$ at $\xi$. As illustrated in Figure 6 , this state is defined by

$$
\left[\operatorname{ev}_{\xi} u\right](\theta):=u(\xi+\theta), \quad \theta \in[-1,1] .
$$

This allows us to define the spaces

$$
\begin{aligned}
P_{f, \lambda} & =\left\{\phi \in C([-1,1], \mathbb{C}) \mid \phi=\mathrm{ev}_{0} v \text { for some } v \in \mathcal{P}_{f, \lambda}\right\}, \\
Q_{f, \lambda} & =\left\{\phi \in C([-1,1], \mathbb{C}) \mid \phi=\mathrm{ev}_{0} v \text { for some } v \in \mathcal{Q}_{f, \lambda}\right\},
\end{aligned}
$$

and their counterparts $P_{b, \lambda}$ and $Q_{b, \lambda}$ for (3.2).

Lemma 3.2. There exists $\delta_{0}>0$ such that the operators $\Lambda_{f, \lambda}$ and $\Lambda_{b, \lambda}$ are Fredholm with index zero for all $\lambda \in \mathbb{C}$ that have $\operatorname{Re} \lambda \geq-\delta_{0}$.

Proof. In view of [MP99a, Thm. A], the operators $\Lambda_{f, \lambda}$ and $\Lambda_{b, \lambda}$ are Fredholm if and only if the characteristic equations

$$
\Delta_{\#}(0,0, \lambda, z)=0, \quad \Delta_{\#}\left(0, w_{*}, \lambda, z\right)=0, \quad \#=L, R,
$$

which are associated to (3.1) and (3.2) in the limits $\xi \rightarrow \pm \infty$, admit no roots $z \in i \mathbb{R}$. This however follows directly from Lemma 3.1. To see that the Fredholm index of $\Lambda_{f, \lambda}$ is zero, imitate the proof of Lemma 3.1 to observe that the equation

$$
s \Delta_{L}(0,0, \lambda, z)+(1-s) \Delta_{R}(0,0, \lambda, z)=0
$$

admits no roots $z \in i \mathbb{R}$ for all $0 \leq s \leq 1$ and invoke the spectral flow result [MP99a, Thm. C]. The fact that $\Lambda_{b, \lambda}$ also has index zero follows in a similar fashion.

Lemma 3.3. There exists $\delta_{0}>0$ such that the identities

$$
\operatorname{dim} \operatorname{Ker} \Lambda_{f, \lambda}=0, \quad \operatorname{dim} \operatorname{Ker} \Lambda_{b, \lambda}=0
$$

and the splittings

$$
C([-1,1], \mathbb{C})=P_{f, \lambda} \oplus Q_{f, \lambda}=Q_{b, \lambda} \oplus Q_{b, \lambda}
$$

hold for any $\lambda \in \mathbb{C} \backslash 2 \pi i c_{*} \mathbb{Z}$ that has $\operatorname{Re} \lambda \geq-\delta_{0}$.

Proof. Recall the constant $\delta_{0}$ from Lemma 3.2 and consider the set

$$
S=\left\{\lambda \in \mathbb{C} \mid \operatorname{Re} \lambda \geq-\delta_{0} \text { and } \operatorname{dim} \operatorname{Ker} \Lambda_{f, \lambda}>0\right\} .
$$

Combining [Hen81, Thm. 5.A.1] and the proof of [CMPS98, Prop 2.2(3)], we see that the set $S$ contains only isolated points. Now consider $\lambda \notin 2 \pi i c_{*} \mathbb{Z}$ and suppose that $\Lambda_{f, \lambda} v=0$ for some nonzero $v \in B C_{0}^{1}(\mathbb{R}, \mathbb{R})$. The lattice function $U_{i}(t)=$ 
$e^{\lambda t} v\left(i+c_{*} t\right)$ then satisfies the linearization (2.17) and has $U_{i}\left(t+c_{*}^{-1}\right)=e^{\lambda / c_{*}} U_{i+1}(t)$, which shows $\mathcal{R} U_{i}=e^{\lambda / c_{*}} U_{i}$. However, [CMPS98, Thm. C] implies that the spectral condition (2.23) must hold, which shows $\operatorname{Re} \lambda<0$. In view of the symmetry (2.9), we can hence decrease $\delta_{0}$ to ensure that (3.21) holds for all $\lambda$ under consideration. The splitting (3.22) follows from MPVL, Thm. 4.3].

For the remainder of this section, we will restrict ourselves to the case $\lambda=0$ and use the shorthands $\Lambda_{f}=\Lambda_{f, 0}, \Lambda_{b}=\Lambda_{b, 0}, \mathcal{P}_{f}=\mathcal{P}_{f, 0}$ and $\mathcal{Q}_{f}=\mathcal{Q}_{f, 0}$. We will need to consider the formal adjoints

$$
\Lambda_{f}^{*}: B C_{0}^{1}(\mathbb{R}, \mathbb{C}) \rightarrow B C_{0}(\mathbb{R}, \mathbb{C}) \quad \text { and } \quad \Lambda_{b}^{*}: B C_{0}^{1}(\mathbb{R}, \mathbb{C}) \rightarrow B C_{0}(\mathbb{R}, \mathbb{C})
$$

that act as

$$
\Lambda_{f}^{*} v=c_{*} v^{\prime}+L\left(q_{f}\right) v \quad \text { and } \quad \Lambda_{b}^{*} v=c_{*} v^{\prime}+L\left(q_{b}\right) v .
$$

We introduce the kernels

$$
\begin{array}{ll}
\mathbb{K}_{f}=\operatorname{Ker} \Lambda_{f}, & \mathbb{K}_{f}^{*}=\operatorname{Ker} \Lambda_{f}^{*}, \\
\mathbb{K}_{b}=\operatorname{Ker} \Lambda_{b}, & \mathbb{K}_{b}^{*}=\operatorname{Ker} \Lambda_{b}^{*},
\end{array}
$$

which are now nonempty since $\Lambda_{f} q_{f}^{\prime}=0$ and $\Lambda_{b} q_{b}^{\prime}=0$. The following description of these kernels follows directly from [MP99b, Theorem 4.1].

Lemma 3.4. Suppose that $(H)$ is satisfied. Then we have $q_{f}^{\prime}(\xi)>0$ and $q_{b}^{\prime}(\xi)<0$ for all $\xi \in \mathbb{R}$, together with

$$
\mathbb{K}_{f}=\operatorname{span}\left\{q_{f}^{\prime}\right\}, \quad \mathbb{K}_{b}=\operatorname{span}\left\{q_{b}^{\prime}\right\} .
$$

In addition, there exist two bounded functions $d_{f}$ and $d_{b}$ that decay exponentially at both $\pm \infty$ and have $d_{f}(\xi)>0$ and $d_{b}(\xi)>0$ for all $\xi \in \mathbb{R}$, such that

$$
\mathbb{K}_{f}^{*}=\operatorname{span}\left\{d_{f}\right\}, \quad \mathbb{K}_{b}^{*}=\operatorname{span}\left\{d_{b}\right\} .
$$

Associated to these kernels, we define the spaces

$$
B_{f}=\operatorname{span}\left\{\operatorname{ev}_{0} q_{f}^{\prime}\right\}, \quad B_{b}=\operatorname{span}\left\{\operatorname{ev}_{0} q_{b}^{\prime}\right\} .
$$

We now find that

$$
P_{f} \cap Q_{f}=B_{f} \quad \text { and } \quad P_{b} \cap Q_{b}=B_{b} .
$$

In view of these nonempty intersections, we introduce the normalized spaces

$$
\begin{aligned}
& \widehat{\mathcal{P}}_{f}=\left\{v \in \mathcal{P}_{f} \mid \int_{-\infty}^{0} q_{f}^{\prime}(\xi) v(\xi) d \xi=0\right\}, \\
& \widehat{\mathcal{Q}}_{f}=\left\{v \in \mathcal{Q}_{f} \mid \int_{+\infty}^{0} q_{f}^{\prime}(\xi) v(\xi) d \xi=0\right\},
\end{aligned}
$$

together with

$$
\begin{aligned}
& \widehat{P}_{f}=\left\{\phi \in C([-1,1], \mathbb{C}) \mid \phi=\mathrm{ev}_{0} v \text { for some } v \in \widehat{\mathcal{P}}_{f}\right\}, \\
& \widehat{Q}_{f}=\left\{\phi \in C([-1,1], \mathbb{C}) \mid \phi=\mathrm{ev}_{0} v \text { for some } v \in \widehat{\mathcal{Q}}_{f}\right\}
\end{aligned}
$$

and their natural counterparts $\widehat{\mathcal{P}}_{b}, \widehat{\mathcal{Q}}_{b}, \widehat{P}_{b}$ and $\widehat{Q}_{b}$.

In order to obtain a splitting for the state space $C([-1,1], \mathbb{C})$ along the lines of (3.22), we need to use the Hale inner product [HVL93]. In the current setting this bilinear form is given by

$$
\langle\psi, \phi\rangle:=c_{*} \psi(0)^{*} \phi(0)-\alpha\left[\int_{0}^{1} \psi(\sigma-1)^{*} \phi(\sigma) \mathrm{d} \sigma+\int_{0}^{-1} \psi(\sigma+1)^{*} \phi(\sigma) \mathrm{d} \sigma\right]
$$


for any pair $\phi, \psi \in C([-1,1], \mathbb{C})$. The Hale inner product is nondegenerate in the sense that if $\langle\psi, \phi\rangle=0$ for all $\psi \in C([-1,1], \mathbb{C})$, then necessarily $\phi=0$ [MPVL]. As a consequence of [MPVL, Thm. 4.3], we have the characterization

$$
\widehat{P}_{f} \oplus \widehat{Q}_{f} \oplus B_{f}=\left\{\phi \in C([-1,1]) \mid\left\langle\mathrm{ev}_{0} d_{f}, \phi\right\rangle=0\right\} .
$$

Let us now pick a one-dimensional space $\Gamma_{f} \subset C([-1,1], \mathbb{C})$ that has the property that $\phi \in \Gamma_{f}$ satisfies $\phi=0$ if and only if $\left\langle\operatorname{ev}_{0} d_{f}, \phi\right\rangle=0$. Picking $\Gamma_{b}$ in a similar fashion, we now see that

$$
C([-1,1], \mathbb{C})=B_{f} \oplus \widehat{Q}_{f} \oplus \widehat{P}_{f} \oplus \Gamma_{f}=B_{b} \oplus \widehat{Q}_{b} \oplus \widehat{P}_{b} \oplus \Gamma_{b} .
$$

As is customary, the homoclinic orbits $\left(q_{f}, 0\right)$ and $\left(q_{b}, w_{*}\right)$ for the travelling wave equation (1.6) break when changing $c$ and $\epsilon$. The key ingredient we will use in this paper is that the arising gaps can be captured in the finite-dimensional spaces $\Gamma_{f}$ and $\Gamma_{b}$. As a consequence, the size of such gaps can be measured effectively by means of the Hale inner product. This is particularly useful in view of the identity

$$
\begin{aligned}
\frac{\mathrm{d}}{\mathrm{d} \xi}\left\langle\operatorname{ev}_{\xi} y, \mathrm{ev}_{\xi} v\right\rangle & =y(\xi)^{*}\left[\Lambda_{f} v\right](\xi)+\left[\Lambda_{f}^{*} y\right](\xi)^{*} v(\xi) \\
& =y(\xi)^{*}\left[\Lambda_{b} v\right](\xi)+\left[\Lambda_{b}^{*} y\right](\xi)^{*} v(\xi),
\end{aligned}
$$

which holds for any pair $y, v \in C^{1}(\mathbb{R}, \mathbb{C})$ and $\xi \in \mathbb{R}$. Indeed, picking $y=d_{f}$ or $y=d_{b}$ and integrating (3.36), we will be able to use Melnikov-type identities to quantify the size of the aforementioned gaps.

\section{Construction of travelling Pulses}

In this section we briefly recall the building blocks that were used in HS10 to construct the travelling pulses described in Theorem 1.1. Our approach towards proving the nonlinear stability of these pulses will proceed along similar lines and rely heavily on the estimates described in the results below. We give a short illustrated sketch of the pulse construction in $\$ 4.1$ and defer the technical details to \$.2 A more comprehensive overview of the material presented here can be found in [HS10, $\S 3]$.

4.1. Sketch of construction. Instead of looking at (1.6) directly, we choose a $C^{\infty}$-smooth cut-off function $\chi_{\mathrm{s} 1}: \mathbb{R} \rightarrow \mathbb{R}$ as shown in Figure 7 and consider the system

$$
\begin{aligned}
& c u^{\prime}(\xi)=\alpha[u(\xi+1)+u(\xi-1)-2 u(\xi)]+g(u(\xi))-w(\xi), \\
& c w^{\prime}(\xi)=\epsilon(u(\xi)-\gamma w(\xi)) \chi_{\mathrm{sl}}(w(\xi)) .
\end{aligned}
$$

This modification allows us to talk about solutions that connect the manifolds $\mathcal{M}_{L}$ and $\mathcal{M}_{R}$ without worrying about the complications that arise when $w$ leaves the region $\left[w_{\min }, w_{\max }\right]$.

Let us first consider what happens to the front $\left(q_{f}, 0\right)$ as the parameters $(c, \epsilon)$ move away from $\left(c_{*}, 0\right)$. In general, we do not expect heteroclinic connections from $(0,0)$ to $\mathcal{M}_{R}$ to persist. However, such connections can in fact be found if we allow them to have a discontinuity in the $u$-component at $\xi=0$. Furthermore, such discontinuous connections are fixed uniquely by the requirement that the jump at $\xi=0$ lies in the one-dimensional space $\Gamma_{f}$ introduced in (3.35). The resulting solutions are called quasi-fronts and are depicted in Figure 8 . We remark that the jump in $\Gamma_{f}$ is of order $O\left(\epsilon+\left|c-c_{*}\right|\right)$. In addition, the signs of the derivatives of this jump with respect to $c$ and $\epsilon$ can be determined by evaluating Melnikov integrals. 


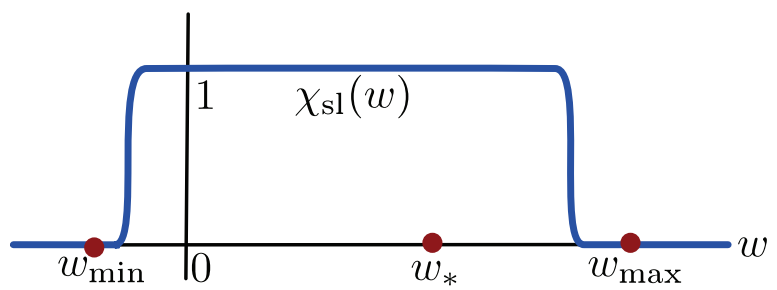

FIGURE 7. The definition of the cut-off function $\chi_{\mathrm{sl}}(w)$.

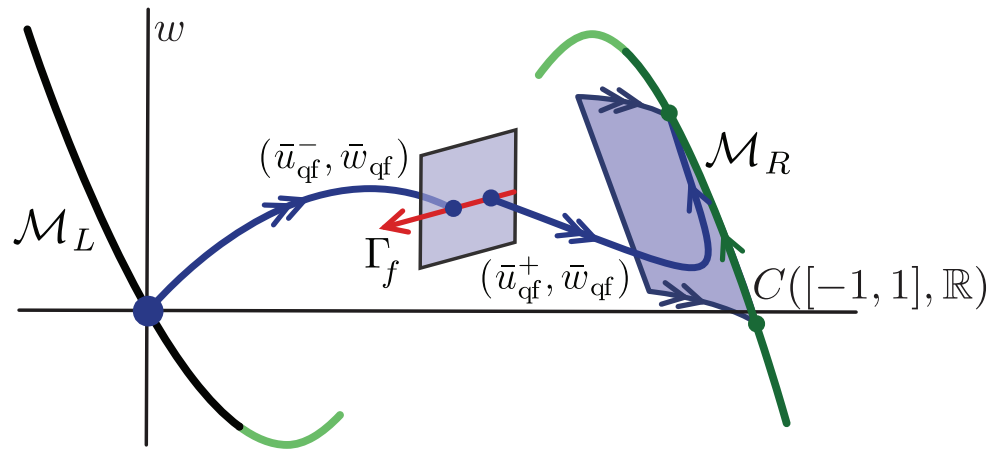

FiguRE 8. Shown is a quasi-front solution which consists of two solutions that lie respectively in the unstable manifold of the equilibrium $(u, w)=0$ and the stable foliation of the slow manifold $\mathcal{M}_{R}$. These solutions will, in general, not coincide but can be chosen so that their difference at $\xi=0$ lies in the one-dimensional subspace $\Gamma_{f}$ of the phase space $C([-1,1], \mathbb{R})$. The new equilibria inside $\mathcal{M}_{R}$ are created by the cut-off function in (4.1).

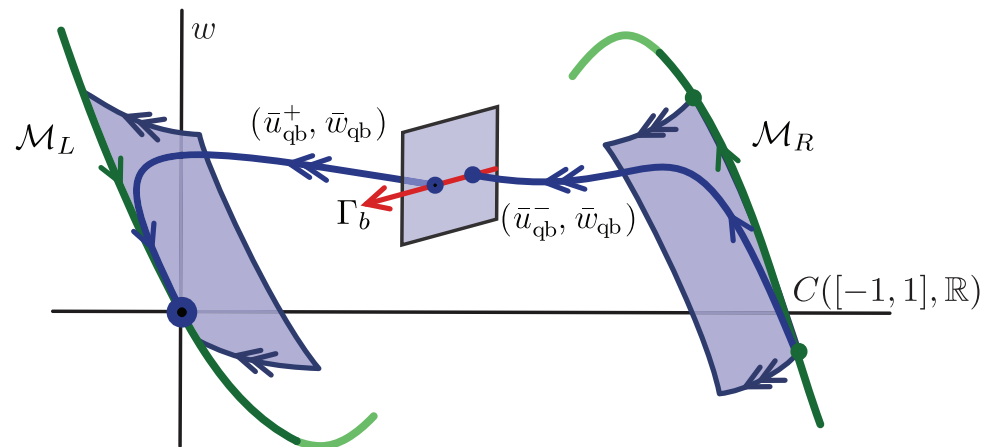

Figure 9. Shown is a quasi-back solution which consists of two solutions that lie respectively in the stable manifold of the slow manifold $\mathcal{M}_{L}$ and the unstable foliation of the slow manifold $\mathcal{M}_{R}$. Compared to the quasi-fronts, there is an additional degree of freedom when constructing quasi-backs as one can choose the stable fibre of $\mathcal{M}_{L}$ to which to converge. 


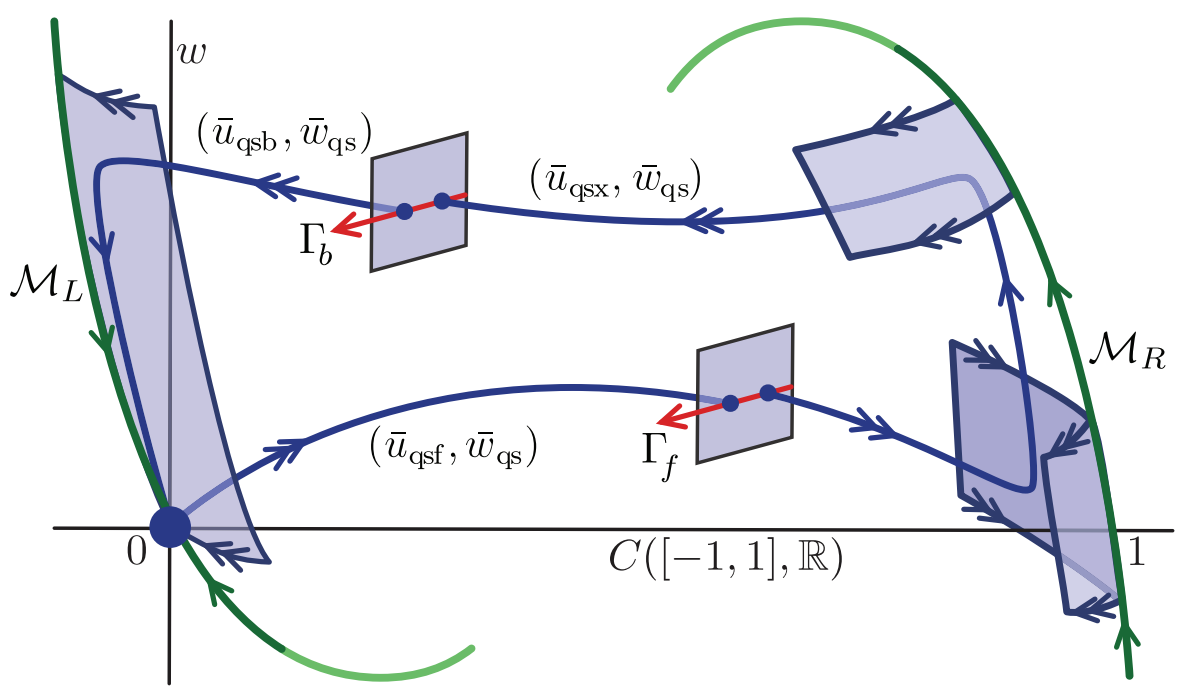

Figure 10. An illustration of quasi-solutions and their passage near the slow manifold $\mathcal{M}_{R}$.

In a similar fashion, a family of discontinuous quasi-back solutions branches off from the back $\left(q_{b}, w_{*}\right)$ as $(c, \epsilon)$ are varied. In this case, there is an additional degree of freedom, as we may move the quasi-back up and down slightly. Depicted in Figure 9, these quasi-backs connect $\mathcal{M}_{R}$ back to $\mathcal{M}_{L}$ and have a jump at $\xi=0$ that now is contained in $\Gamma_{b}$. This time, the jump is of order $O\left(\epsilon+\left|c-c_{*}\right|+\left|w(0)-w_{*}\right|\right)$.

Using an infinite-dimensional version of the Exchange Lemma, the quasi-fronts and quasi-backs can be glued together near $\mathcal{M}_{R}$ at the price of two additional jumps that can also be contained in the spaces $\Gamma_{f}$ and $\Gamma_{b}$. The resulting quasi-solutions are depicted in Figure 10.

We now have three free parameters in the problem and two one-dimensional jumps that need to be closed. The contribution from the Exchange Lemma is $C^{1}$-exponentially small with respect to the time spent near $\mathcal{M}_{R}$, which is of order $O(1 / \epsilon)$. This contribution can hence safely be neglected and the remaining equations can be solved to yield the one-parameter branch of solution described in Theorem 1.1 .

4.2. Summary of results from HS10. In this part we recall the key technical results that were obtained in [HS10] and present them in a form that will be useful for our analysis in 55+6. To simplify our notation, we will depart from the setting in [HS10, §3] and assume a priori knowledge of the wave speeds $c(\epsilon)$ here. We hence study the system

$$
\begin{aligned}
& c(\epsilon) u^{\prime}(\xi)=\alpha[u(\xi+1)+u(\xi-1)-2 u(\xi)]+g(u(\xi))-w(\xi), \\
& c(\epsilon) w^{\prime}(\xi)=\epsilon(u(\xi)-\gamma w(\xi)) \chi_{\mathrm{sl}}(w(\xi)) .
\end{aligned}
$$

We start by studying the behaviour of the equilibrium manifold $\mathcal{M}$ as $\epsilon$ moves away from zero. Our first result shows how the invariant manifolds $\mathcal{M}_{L}$ and $\mathcal{M}_{R}$ persist for $\epsilon>0$. 
Proposition 4.1 (See HS10, §4]). Consider the nonlinear system (4.2). There exists a constant $\delta_{\epsilon}>0$ together with two $C^{\infty}$-smooth functions

$$
s_{R}, s_{L}:\left[w_{\min }, w_{\max }\right] \times\left[0, \delta_{\epsilon}\right] \rightarrow \mathbb{R}
$$

that satisfy the following properties.

(i) For each $\vartheta \in\left[w_{\min }, w_{\max }\right]$ and $\epsilon \in\left[0, \delta_{\epsilon}\right]$, we have

$$
s_{R}(\vartheta, 0)=\widetilde{s}_{R}(\vartheta) \quad \text { and } \quad s_{L}(\vartheta, 0)=\widetilde{s}_{L}(\vartheta) .
$$

(ii) For each $\vartheta \in\left[w_{\min }, w_{\max }\right]$ and $\epsilon \in\left[0, \delta_{\epsilon}\right]$, the unique solution of the $O D E$

$$
c(\epsilon) w^{\prime}(\xi)=\epsilon\left(s_{R}(w(\xi), \epsilon)-\gamma w(\xi)\right) \chi_{\mathrm{sl}}(w(\xi)), \quad w(0)=\vartheta,
$$

yields a solution $\left(s_{R}(w, \epsilon), w\right)$ to (4.2). The same statement holds upon replacing the subscript $R$ by $L$.

(iii) There exists a constant $\delta>0$ such that any solution $(u, w)$ to (4.2) with $0 \leq \epsilon \leq \delta_{\epsilon}$ that has both $w_{\min } \leq w(\xi) \leq w_{\max }$ and $\left|u(\xi)-\widetilde{s}_{R}(w(\xi))\right|<\delta$ for all $\xi \in \mathbb{R}$ must in fact satisfy $u(\xi)=s_{R}(w(\xi), \epsilon)$ for all $\xi \in \mathbb{R}$. The same statement holds for the subscript $L$.

Our second result describes the quasi-front and quasi-back solutions to (4.2) depicted in Figures 8 and 9 . As explained in $\$ 4.1$, these solutions should be seen as orbits that bifurcate from $q_{f}$ and $q_{b}$ and connect $\mathcal{M}_{L}$ to $\mathcal{M}_{R}$ and vice versa, at the price of a discontinuity at $\xi=0$ that can be contained in the spaces $\Gamma_{f}$ and $\Gamma_{b}$. As a preparation, we introduce the notation

$$
\widehat{X}_{f}=\widehat{Q}_{f} \oplus \widehat{P}_{f} \oplus \Gamma_{f} \quad \text { and } \quad \widehat{X}_{b}=\widehat{Q}_{b} \oplus \widehat{P}_{b} \oplus \Gamma_{b}
$$

and recall the exponent $\eta_{*}$ that appears in Lemma 3.1

Proposition 4.2 (See [HS10, §5]). Pick a sufficiently large constant $\xi_{*}>0$ and a sufficiently small constant $\delta_{\tilde{\epsilon}}>0$. Then for every pair $\left(\xi_{0}, \epsilon\right)$ with $\xi_{0} \geq \xi_{*}$ and $0 \leq \epsilon \xi_{0} \leq \delta_{\tilde{\epsilon}}$, there exists a set of functions

$$
\begin{array}{ll}
\bar{u}_{\mathrm{qf}}^{-}\left(\xi_{0}, \epsilon\right), & \bar{u}_{\mathrm{qb}}^{-}\left(\xi_{0}, \epsilon\right) \in C((-\infty, 1], \mathbb{R}), \\
\bar{u}_{\mathrm{qf}}^{+}\left(\xi_{0}, \epsilon\right), & \bar{u}_{\mathrm{qb}}^{+}\left(\xi_{0}, \epsilon\right) \in C([-1, \infty), \mathbb{R}), \\
\bar{w}_{\mathrm{qf}}\left(\xi_{0}, \epsilon\right), & \bar{w}_{\mathrm{qb}}\left(\xi_{0}, \epsilon\right) \in C(\mathbb{R}, \mathbb{R})
\end{array}
$$

that satisfies the following properties.

(i) The pairs $\left(\bar{u}_{\mathrm{qf}}^{ \pm}, \bar{w}_{\mathrm{qf}}\right)$ and $\left(\bar{u}_{\mathrm{qb}}^{ \pm}, \bar{w}_{\mathrm{qb}}\right)$ satisfy (4.2) on the intervals $\mathbb{R}_{ \pm}$.

(ii) We have the inclusions

$$
\begin{array}{llll}
\mathrm{ev}_{0} \bar{u}_{\mathrm{qf}}^{-}\left(\xi_{0}, \epsilon\right) & \in \mathrm{ev}_{0} q_{f}+\widehat{X}_{f}, & \mathrm{ev}_{0} \bar{u}_{\mathrm{qf}}^{+}\left(\xi_{0}, \epsilon\right) & \in \mathrm{ev}_{0} q_{f}+\widehat{X}_{f}, \\
\mathrm{ev}_{0} \bar{u}_{\mathrm{qb}}^{-}\left(\xi_{0}, \epsilon\right) \in \mathrm{ev}_{0} q_{b}+\widehat{X}_{b}, & \mathrm{ev}_{0} \bar{u}_{\mathrm{qb}}^{+}\left(\xi_{0}, \epsilon\right) \in & \mathrm{ev}_{0} q_{b}+\widehat{X}_{b} .
\end{array}
$$

(iii) We have the jump conditions

$$
\mathrm{ev}_{0} \bar{u}_{\mathrm{qf}}^{-}\left(\xi_{0}, \epsilon\right)-\mathrm{ev}_{0} \bar{u}_{\mathrm{qf}}^{+}\left(\xi_{0}, \epsilon\right) \in \Gamma_{f}, \quad \mathrm{ev}_{0} \bar{u}_{\mathrm{qb}}^{-}\left(\xi_{0}, \epsilon\right)-\mathrm{ev}_{0} \bar{u}_{\mathrm{qb}}^{+}\left(\xi_{0}, \epsilon\right) \in \Gamma_{b} .
$$

(iv) Choose any $\xi_{0} \geq \xi_{*}$. Restricting $q_{f}$ and $q_{b}$ to the appropriate intervals, we have $\bar{u}_{\mathrm{qf}}^{ \pm}\left(\xi_{0}, 0\right)=q_{f}$ and $\bar{u}_{\mathrm{qb}}^{ \pm}\left(\xi_{0}, 0\right)=q_{b}$. In addition, we have $\bar{w}_{\mathrm{qf}}\left(\xi_{0}, 0\right)=0$ and $\bar{w}_{\mathrm{qb}}\left(\xi_{0}, 0\right)=w_{*}$. 
(v) Choose any $\xi_{0} \geq \xi_{*}$. Upon taking the appropriate restrictions, the maps

$$
\tilde{\epsilon} \mapsto\left\{\begin{array}{lll}
\bar{u}_{\mathrm{qf}}^{-}\left(\xi_{0}, \tilde{\epsilon} / \xi_{0}\right) & \in B C_{-\eta_{*}}((-\infty, 1], \mathbb{R}), \\
\bar{u}_{\mathrm{qf}}^{+}\left(\xi_{0}, \widetilde{\epsilon} / \xi_{0}\right) & \in B C_{0}\left(\left[-1, \xi_{0}\right], \mathbb{R}\right), \\
e^{-\eta_{*} \xi_{0}}\left[\bar{u}_{\mathrm{ff}}^{+}\left(\xi_{0}, \tilde{\epsilon} / \xi_{0}\right)-s_{R}\left(\bar{w}_{\mathrm{qf}}\left(\xi_{0}, \widetilde{\epsilon} / \xi_{0}\right), \widetilde{\epsilon} / \xi_{0}\right)\right] & \in B C_{-\eta_{*}}\left(\left[\xi_{0}, \infty\right), \mathbb{R}\right), \\
\bar{w}_{\mathrm{qf}}\left(\xi_{0}, \tilde{\epsilon} / \xi_{0}\right) & \in B C_{-\eta_{*}}((-\infty, 0], \mathbb{R}), \\
\bar{w}_{\mathrm{qf}}\left(\xi_{0}, \tilde{\epsilon} / \xi_{0}\right) & \in B C_{0}\left(\left[0, \xi_{0}\right], \mathbb{R}\right), \\
e^{\nu \xi_{0}} \bar{w}_{\mathrm{qf}}\left(\xi_{0}, \widetilde{\epsilon} / \xi_{0}\right) & \in B C_{\nu}\left(\left[\xi_{0}, \infty\right), \mathbb{R}\right)
\end{array}\right.
$$

$$
\widetilde{\epsilon} \mapsto\left\{\begin{array}{lll}
e^{-\eta_{*} \xi_{0}}\left[\bar{u}_{\mathrm{qb}}^{-}\left(\xi_{0}, \tilde{\epsilon} / \xi_{0}\right)-s_{R}\left(\bar{w}_{\mathrm{qb}}\left(\xi_{0}, \tilde{\epsilon} / \xi_{0}\right), \tilde{\epsilon} / \xi_{0}\right)\right] & \in B C_{-\eta_{*}}\left(\left(-\infty,-\xi_{0}\right], \mathbb{R}\right), \\
\bar{u}_{\mathrm{qb}}^{-}\left(\xi_{0}, \widetilde{\epsilon} / \xi_{0}\right) & \in B C_{0}\left(\left[-\xi_{0}, 1\right], \mathbb{R}\right), \\
\bar{u}_{\mathrm{qb}}^{+}\left(\xi_{0}, \tilde{\epsilon} / \xi_{0}\right) & \in B C_{0}\left(\left[-1, \xi_{0}\right], \mathbb{R}\right), \\
e^{-\eta_{*} \xi_{0}}\left[\bar{u}_{\mathrm{qb}}^{+}\left(\xi_{0}, \widetilde{\epsilon} / \xi_{0}\right)-s_{L}\left(\bar{w}_{\mathrm{qb}}\left(\xi_{0}, \widetilde{\epsilon} / \xi_{0}\right), \widetilde{\epsilon} / \xi_{0}\right)\right] & \in B C_{-\eta_{*}}\left(\left[\xi_{0}, \infty\right), \mathbb{R}\right), \\
e^{\nu \xi_{0}} \bar{w}_{\mathrm{qb}}\left(\xi_{0}, \widetilde{\epsilon} / \xi_{0}\right) & \in B C_{\nu}\left(\left(-\infty,-\xi_{0}\right], \mathbb{R}\right), \\
\bar{w}_{\mathrm{qb}}\left(\widetilde{\epsilon} / \xi_{0}\right) & \in B C_{0}\left(\left[-\xi_{0}, \xi_{0}\right], \mathbb{R}\right), \\
e^{\nu \xi_{0}} \bar{w}_{\mathrm{qb}}\left(\xi_{0}, \tilde{\epsilon} / \xi_{0}\right) & \in B C_{\nu}\left(\left[\xi_{0}, \infty\right), \mathbb{R}\right)
\end{array}\right.
$$

are all $C^{2}$-smooth with derivatives that can be bounded independently of $\xi_{0} \geq \xi_{*}$. Here $\nu$ is a constant of order $\nu=O\left(\delta_{\tilde{\epsilon}} / \xi_{*}\right)$.

We remark that the exponential terms appearing in property (v) above merely serve to counteract the effects of the weight in (2.53) on intervals that start at $\pm \xi_{0}$.

Our third result describes the correction terms that are necessary to join together the quasi-fronts and quasi-backs. Due to the singular nature of the limit $\epsilon \rightarrow 0$, it turns out to be fruitful to introduce new variables $T^{\mathrm{sl}}$ and $T$ that measure the time needed to travel between the Poincaré sections $\mathrm{ev}_{0} q_{f}+\widehat{X}_{f}$ and $\mathrm{ev}_{0} q_{b}+\widehat{X}_{b}$ in the slow and fast time scales. These time scales are related via the identification

$$
T^{\mathrm{sl}}=\epsilon T .
$$

We choose $T_{*}^{\text {sl }}$ in such a way that the unique solution to the ODE

$$
c_{*} w^{\prime}(\zeta)=\widetilde{s}_{R}(\zeta)-\gamma w(\zeta), \quad w(0)=0
$$

has $w\left(T_{*}^{\mathrm{sl}}\right)=w_{*}$. This ODE corresponds to the flow along $\mathcal{M}_{R}$ in the slow time scale and hence $T_{*}^{\text {sl }}$ can be interpreted as the slow time spent on the segment of the singular orbit $\Gamma_{0}$ that is contained in the slow manifold $\mathcal{M}_{R}$.

Treating $T^{\mathrm{sl}}$ and $T$ as independent variables, we will be interested in the parameter space

$$
\Omega=\Omega\left(\xi_{*}, \delta_{\mathrm{sl}}, M_{T}\right)=\left\{\left(\xi_{0}, T^{\mathrm{sl}}, T\right) \mid \xi_{0} \geq \xi_{*} \text { and }\left|T^{\mathrm{sl}}-T_{*}^{\mathrm{sl}}\right|<\delta_{\lambda} \text { and } T \geq M_{T} \xi_{0}\right\} .
$$

For any $\omega \in \Omega$, the result below shows that a quasi-solution for (4.2) can be constructed that is $C^{1}$-exponentially close to the corresponding quasi-front and quasiback with respect to the time $T$. Such a quasi-solution is illustrated in Figure 10,

Proposition 4.3 (See [HS10, §6]). Pick sufficiently large constants $\xi_{*}>0, M_{T}>0$ and a sufficiently small constant $\delta_{\mathrm{sl}}>0$. Then for every

$$
\omega=\left(\xi_{0}, T^{\mathrm{sl}}, T\right) \in \Omega=\Omega\left(\xi_{*}, \delta_{\mathrm{sl}}, M_{T}\right),
$$


there exists a quadruplet $\left(\bar{u}_{\mathrm{qsf}}(\omega), \bar{u}_{\mathrm{qsb}}(\omega), \bar{u}_{\mathrm{qsx}}(\omega), \bar{w}_{\mathrm{qs}}(\omega)\right)$ with

$$
\begin{array}{ll}
\bar{u}_{\mathrm{qsf}}(\omega) & \in C((-\infty, 1], \mathbb{R}), \\
\bar{u}_{\mathrm{qsb}}(\omega) & \in C([T-1, \infty), \mathbb{R}), \\
\bar{u}_{\mathrm{qsx}}(\omega) & \in C([-1, T+1], \mathbb{R}), \\
\bar{w}_{\mathrm{qs}}(\omega) & \in C(\mathbb{R}, \mathbb{R})
\end{array}
$$

that satisfies the following properties.

(i) The pair $\left(\bar{u}_{\mathrm{qsf}}(\omega), \bar{w}_{\mathrm{qs}}(\omega)\right)$ satisfies (4.2) on the interval $(\infty, 0]$, the pair $\left(\bar{u}_{\mathrm{qsx}}(\omega), \bar{w}_{\mathrm{qs}}(\omega)\right)$ satisfies (4.2) on $[0, T]$ and the pair $\left(\bar{u}_{\mathrm{qsb}}(\omega), \bar{w}_{\mathrm{qs}}(\omega)\right)$ satisfies (4.2) on $[T, \infty)$.

(ii) We have the inclusions

$$
\begin{array}{lll}
\operatorname{ev}_{0} \bar{u}_{\mathrm{qsf}}(\omega) \in \mathrm{ev}_{0} q_{f}+\widehat{X}_{f}, & \operatorname{ev}_{0} \bar{u}_{\mathrm{qsx}}(\omega) \in \mathrm{ev}_{0} q_{f}+\widehat{X}_{f}, \\
\operatorname{ev}_{T} \bar{u}_{\mathrm{qsx}}(\omega) \in \mathrm{ev}_{0} q_{b}+\widehat{X}_{b}, & \operatorname{ev}_{T} \bar{u}_{\mathrm{qsb}}(\omega) \in \mathrm{ev}_{0} q_{b}+\widehat{X}_{b} .
\end{array}
$$

(iii) We have the jump conditions

$$
\mathrm{ev}_{0} \bar{u}_{\mathrm{qsf}}(\omega)-\mathrm{ev}_{0} \bar{u}_{\mathrm{qsx}}(\omega) \in \Gamma_{f}, \quad \mathrm{ev}_{T} \bar{u}_{\mathrm{qsx}}(\omega)-\mathrm{ev}_{T} \bar{u}_{\mathrm{qsb}}(\omega) \in \Gamma_{b} .
$$

(iv) Taking the appropriate restrictions, the maps

$$
\omega \mapsto \bar{U}(\omega)=\left\{\begin{array}{lll}
\bar{u}_{\mathrm{qsf}}(\omega)-\bar{u}_{\mathrm{qf}}(\omega) & \in & B C_{-\eta_{*}}((-\infty, 1], \mathbb{R}), \\
\bar{u}_{\mathrm{qsx}}(\omega)-\bar{u}_{\mathrm{qf}}(\omega) & \in B C_{\eta_{*}}\left(\left[-1, \frac{1}{2} T\right], \mathbb{R}\right), \\
{\left[\bar{u}_{\mathrm{qsx}}(\omega)-\bar{u}_{\mathrm{pb}}(\omega)(\cdot-T)\right] e^{\eta_{*} T}} & \in B C_{\eta_{*}}\left(\left[\frac{1}{2} T, T+1\right], \mathbb{R}\right), \\
{\left[\bar{u}_{\mathrm{qsb}}(\omega)-\bar{u}_{\mathrm{qb}}(\omega)(\cdot-T)\right] e^{-\eta_{*} T}} & \in & B C_{-\eta_{*}}([T-1, \infty), \mathbb{R}),
\end{array}\right.
$$

together with

$$
\omega \mapsto \bar{W}(\omega)=\left\{\begin{array}{lll}
\bar{w}_{\mathrm{qs}}(\omega)-\bar{w}_{\mathrm{qf}}\left(T^{\mathrm{sl}} / T\right) & \in B C_{-\eta_{*}}((-\infty, 0], \mathbb{R}), \\
\bar{w}_{\mathrm{qs}}(\omega)-\bar{w}_{\mathrm{qf}}\left(T^{\mathrm{sl}} / T\right) & \in B C_{\eta_{*}}\left(\left[0, \frac{1}{2} T\right], \mathbb{R}\right), \\
{\left[\bar{w}_{\mathrm{qs}}(\omega)-\bar{w}_{\mathrm{qb}}\left(T^{\mathrm{sl}} / T\right)(\cdot-T)\right] e^{\eta_{*} T}} & \in & B C_{\eta_{*}}\left(\left[\frac{1}{2} T, T\right], \mathbb{R}\right), \\
{\left[\bar{w}_{\mathrm{qs}}(\omega)-\bar{w}_{\mathrm{qb}}\left(T^{\mathrm{sl}} / T\right)(\cdot-T)\right] e^{-\eta_{*} T}} & \in B C_{-\eta_{*}}([T, \infty), \mathbb{R}),
\end{array}\right.
$$

are $C^{1}$-smooth with respect to $T^{\mathrm{sl}}$. In addition, there exists $C>0$ such that the estimates

$$
\begin{aligned}
\|\bar{U}(\omega)\|+\|\bar{W}(\omega)\| & \leq C e^{-\eta_{*} T}, \\
\left\|D_{T^{\mathrm{sl}}} \bar{U}(\omega)\right\|+\left\|D_{T^{\mathrm{sl}}} \bar{W}(\omega)\right\| & \leq C e^{\nu T} e^{-\eta_{*} T}
\end{aligned}
$$

hold for all $\omega \in \Omega$ and integers $0 \leq \ell \leq r$. Here $\nu>0$ is a constant of order $\nu=O\left(\left(\xi_{*} M_{T}\right)^{-1}\right)$.

Our final result shows that for all sufficiently small $\epsilon>0$, the parameter $\omega$ can be chosen in such a way that the jumps mentioned in property (iii) above actually vanish. This yields the branch of solutions described in Theorem 1.1 .

Proposition 4.4 (See HS10, §3.4]). Pick any sufficiently large $\xi_{*}>0, M_{T}>0$ and a sufficiently small $\delta_{\mathrm{sl}}>0$. For any $\xi_{0} \geq \xi_{*}$, there exists a $\delta_{\epsilon}\left(\xi_{0}\right)>0$ and a $C^{1}$-smooth function $T_{\mathrm{nl}}:\left(0, \delta_{\epsilon}\right] \rightarrow \mathbb{R}$ such that the map

$$
\omega_{\mathrm{nl}}:\left(0, \delta_{\epsilon}\left(\xi_{0}\right)\right] \rightarrow \Omega, \quad \epsilon \mapsto\left(\xi_{0}, \epsilon T_{\mathrm{nl}}(\epsilon), T_{\mathrm{nl}}(\epsilon)\right)
$$

is well defined. Furthermore, for each $0<\epsilon<\min \left\{\delta_{\epsilon}\left(\xi_{0}\right), \delta_{\epsilon}\right\}$, the travelling pulse $(\bar{u}, \bar{w})(\epsilon)$ coincides with the quasi-solution $\left(\bar{u}_{\mathrm{qsf}}, \bar{u}_{\mathrm{qsx}}, \bar{u}_{\mathrm{qsb}}, \bar{w}_{\mathrm{qs}}\right)\left(\omega_{\mathrm{nl}}(\epsilon)\right)$, where the constant $\delta_{\epsilon}$ and the branch of solutions $(\bar{u}, \bar{w})(\epsilon)$ were defined in Theorem 1.1 . 


\section{Spectral stability}

In order to establish our main result, Theorem 1.2, we will need to study the operators

$$
\mathcal{L}(\epsilon)-\lambda: B C_{0}^{1}\left(\mathbb{R}, \mathbb{C}^{2}\right) \rightarrow B C_{0}\left(\mathbb{R}, \mathbb{C}^{2}\right)
$$

that act as

$$
[\mathcal{L}(\epsilon)-\lambda](u, w)=-c(\epsilon)\left(u^{\prime}, w^{\prime}\right)+(L(\bar{u}(\epsilon)) u-w, \epsilon(u-\gamma w))-\lambda(u, w) .
$$

In view of Proposition 2.1, it suffices to establish the following result.

Proposition 5.1 (Spectral stability). Consider the setting of Theorem 1.1. Then for any sufficiently small $\epsilon>0$, the operator $\mathcal{L}(\epsilon)$ satisfies conditions (S1) through (S3) in $\oint_{2}$. In particular, the eigenvalues at $\lambda \in 2 \pi i c(\epsilon) \mathbb{Z}$ are simple and isolated and there is no additional spectrum in the half-plane $\operatorname{Re} \lambda \geq-\delta_{0}(\epsilon)$ for some $\delta_{0}(\epsilon)>$ 0 .

In this section we outline our proof of this result, leaving some of the technical details to 96 In $\$ 5.1$ we study the essential spectrum $\sigma_{\text {ess }}(\mathcal{L}(\epsilon))$ and show that it lies to the left of the line $\operatorname{Re} \lambda=-\gamma \epsilon$. In $\$ 5.2$ we characterize the part of the point spectrum $\sigma_{p}(\mathcal{L}(\epsilon))$ that lies to the right of the line $\operatorname{Re} \lambda=-\delta_{0}$, for some small constant $\delta_{0}>0$ that will not depend on $\epsilon$.

In the limiting case $\epsilon=0$, the entire imaginary axis is contained in the essential spectrum. This means that our region of interest $\operatorname{Re} \lambda \geq-\delta_{0}$ will always contain part of the essential spectrum if $\epsilon>0$ is sufficiently small. As a consequence, it is very hard to perform any direct search for eigenvalues that works uniformly for all small $\epsilon>0$.

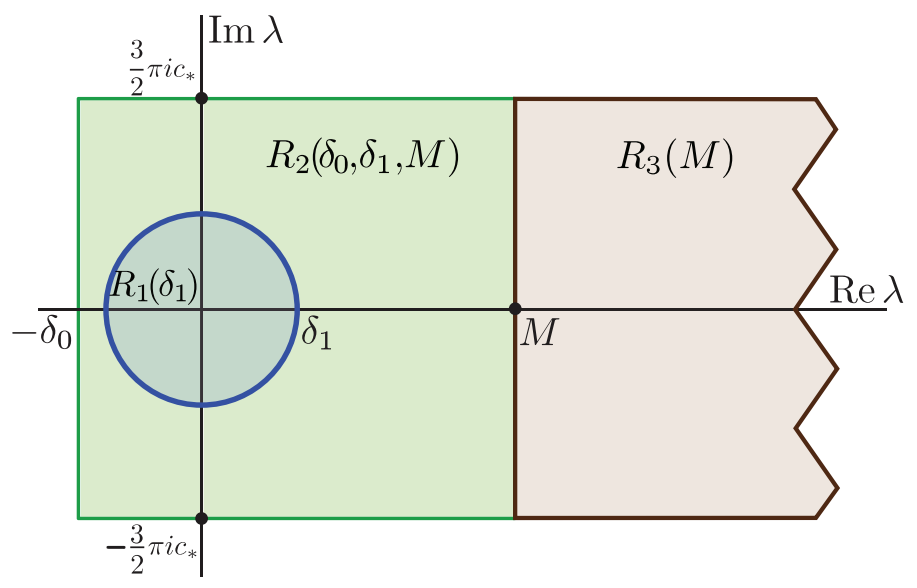

FiguRE 11. Illustration of the regions $R_{1}, R_{2}$ and $R_{3}$. Besides $\lambda=0 \in R_{1}$, we expect to find a second potential eigenvalue in $R_{1}$.

To avoid this problem, we will look for solutions to $\mathcal{L}(\epsilon) v=\lambda v$ that behave as $v(\xi)=O\left(e^{2 \eta \xi}\right)$ as $\xi \rightarrow \pm \infty$, for some appropriate exponent $\eta>0$. If $\lambda$ lies to the right of the essential spectrum, one may show that $v$ is bounded and thus an actual eigenfunction. On the other hand, $v$ may grow exponentially as $\xi \rightarrow \infty$ if $\lambda$ lies to the left of the essential spectrum, in which case we refer to $\lambda$ as a resonance pole. 
The translational invariance of the travelling wave system (1.6) implies that $\lambda=0$ is always an eigenvalue. Since we are gluing a front and a back together, we expect the presence of a second potential eigenvalue $\lambda_{2} \approx 0$. In $\$ 5.2$ we calculate the speed with which $\lambda_{2}$ moves to the left as $\epsilon$ increases. Whether or not $\lambda_{2}$ is an actual eigenvalue depends on how this speed compares to the rate at which the essential spectrum moves into the left half-plane, which is computed in $\$ 5.1$

5.1. Essential spectrum. We set out to describe the essential spectrum $\sigma_{\text {ess }}(\mathcal{L}(\epsilon))$. Note first that the limits

$$
\lim _{\xi \rightarrow \pm \infty}(\bar{u}(\epsilon), \bar{w}(\epsilon))(\xi)=(0,0),
$$

together with [MP99a, Thm. C], imply that the Fredholm index of $\mathcal{L}(\epsilon)-\lambda$ is automatically zero if $\mathcal{L}(\epsilon)-\lambda$ is in fact Fredholm. In view of [MP99a, Thm. A] this is the case if and only if the characteristic equation

$$
\operatorname{det} \Delta_{\mathcal{L}(\epsilon)}(\lambda, z)=0
$$

admits no roots $z \in i \mathbb{R}$. Here the characteristic function $\Delta_{\mathcal{L}(\epsilon)}$ is given by

$$
\Delta_{\mathcal{L}(\epsilon)}(\lambda, z)=\left(\begin{array}{cc}
\Delta_{L}(\epsilon, 0, \lambda, z) & 1 \\
-\epsilon & c(\epsilon) z+\lambda+\gamma \epsilon
\end{array}\right) .
$$

Setting out to locate the roots of (5.4), we will establish the following result.

Proposition 5.2. There exist constants $\delta_{0}>0, \delta_{\epsilon}>0$ and $0<\eta<\frac{1}{4} \eta_{*}$ such that the following properties hold.

(i) For every $\epsilon \in\left[0, \delta_{\epsilon}\right]$, we have

$$
\sigma_{\text {ess }}(\mathcal{L}(\epsilon)) \subseteq\{\lambda \in \mathbb{C} \mid \operatorname{Re} \lambda \leq-\gamma \epsilon\} .
$$

(ii) Choose any $0<\epsilon \leq \delta_{\epsilon}$ and pick any $\lambda \in \mathbb{C}$ that has $\operatorname{Re} \lambda>-\gamma \epsilon$. Then the characteristic equation $\operatorname{det} \Delta_{\mathcal{L}(\epsilon)}(\lambda, z)=0$ admits no roots with $0 \leq \operatorname{Re} z \leq$ $4 \eta$.

(iii) Writing $\lambda_{\text {ess }}(\nu, \epsilon)$ for the rightmost $\lambda \in \mathbb{C}$ that has $\operatorname{det} \Delta_{\mathcal{L}(\epsilon)}(\lambda, i \nu)=0$, we have the expansion

$$
\lambda_{\mathrm{ess}}(\nu, \epsilon)=-i \nu c-\epsilon\left(\gamma+\frac{1}{a+2 \alpha(1-\cos \nu)}\right)+O\left(\epsilon^{2}\right), \quad \nu \in \mathbb{R} .
$$

We start by establishing (i) in the result above. This actually follows from Lemma 3.1 after a very simple computation.

Lemma 5.3. Recall the constants $\delta_{0}>0, \delta_{\epsilon}>0$ and $\eta_{*}>0$ that appear in Lemma 3.1 .

Suppose that $\operatorname{det} \Delta_{\mathcal{L}(\epsilon)}(\lambda, z)=0$ for some $\epsilon \in\left[0, \delta_{\epsilon}\right], \lambda \in \mathbb{C}$ that has $\operatorname{Re} \lambda \geq-\delta_{0}$ and $z \in \mathbb{C}$ that has $|\operatorname{Re} z| \leq \eta_{*}$. Then we have the inequality

$$
\operatorname{Re} \lambda \leq-\gamma \epsilon-c(\epsilon) \operatorname{Re} z .
$$

Proof. Solving the characteristic equation (5.4), we may compute

$$
\begin{aligned}
\operatorname{Re} \lambda & =-\gamma \epsilon-\operatorname{Re} \Delta_{L}(\epsilon, 0, \lambda, z)^{-1} \epsilon-c(\epsilon) \operatorname{Re} z \\
& =-\gamma \epsilon-\frac{\epsilon}{\left|\Delta_{L}(\epsilon, 0, \lambda z)\right|^{2}} \operatorname{Re} \Delta_{L}(\epsilon, 0, \lambda, z)-c(\epsilon) \operatorname{Re} z \\
& \leq-\gamma \epsilon-c(\epsilon) \operatorname{Re} z .
\end{aligned}
$$


In order to establish (ii) in Proposition [5.2, we need to track the behaviour of the root $z=\lambda / c_{*}$ as $\epsilon$ moves away from zero.

Lemma 5.4. There exist constants $\delta_{\epsilon}>0,0<\delta_{0}<\delta_{0}^{*}$ and $0<\eta_{1}<\eta_{2}$, together with a smooth function

$$
z_{*}:\left\{(\lambda, \epsilon) \in \mathbb{C} \times\left[0, \delta_{\epsilon}\right]:|\operatorname{Re} \lambda| \leq \delta_{0}^{*}\right\} \rightarrow \mathbb{C}
$$

that has $z_{*}(\lambda, 0)=-\lambda / c_{*}$, such that the following holds true.

Suppose that $\operatorname{det} \Delta_{\mathcal{L}(\epsilon)}(\lambda, z)=0$ for some $\epsilon \in\left[0, \delta_{\epsilon}\right]$ and some pair $\lambda, z \in \mathbb{C}$ that has $\operatorname{Re} \lambda \geq-\delta_{0}$ and $-\eta_{1} \leq|\operatorname{Re} z| \leq \eta_{2}$. Then we must also have $\operatorname{Re} \lambda \leq \delta_{0}^{*}$, $\operatorname{Re} z \leq \eta_{1}$ and

$$
z=z_{*}(\lambda, \epsilon)
$$

Proof. Recall the constants $\delta_{0}>0, \delta_{\epsilon}>0$ and $\eta_{*}>0$ that appear in Lemma 3.1 Pick any $\lambda_{0} \in i \mathbb{R}$ and note that $\Delta_{L}\left(\epsilon, 0, \lambda_{0}, z\right)=0$ has no roots in the strip $|\operatorname{Re} z| \leq$ $\eta_{*}$ for $\epsilon \in\left[0, \delta_{\epsilon}\right]$. In view of the factorization

$$
\operatorname{det} \Delta_{\mathcal{L}(0)}\left(\lambda_{0}, z\right)=\Delta_{L}\left(0,0, \lambda_{0}, z\right)\left(c_{*} z+\lambda_{0}\right),
$$

we may conclude that $z=-\lambda_{0} / c_{*} \in i \mathbb{R}$ is the unique root of the characteristic equation

$$
\operatorname{det} \Delta_{\mathcal{L}(0)}\left(\lambda_{0}, z\right)=0
$$

that has $|\operatorname{Re} z| \leq \eta_{*}$. Using [HVL07, Lem. 3.1], we note that the imaginary part of any root $z$ of the characteristic equation (5.4) can be a priori bounded if $\lambda$ and $\epsilon$ are restricted to compact intervals. This allows us to find $\delta_{\lambda}>0$ such that the characteristic equation

$$
\operatorname{det} \Delta_{\mathcal{L}(\epsilon)}\left(\lambda_{0}+\lambda, z\right)=0
$$

admits no roots with $|\operatorname{Re} z|=\frac{1}{2} \eta_{*}$ for any $|\lambda|<\delta_{\lambda}$ and any $\epsilon \in\left[0, \delta_{\epsilon}\right]$, possibly after decreasing $\delta_{\epsilon}$. This allows us to define $z_{*}(\lambda, \epsilon)$ as the unique root of (5.4) in the strip $|\operatorname{Re} z| \leq \frac{1}{2} \eta_{*}$, for all $(\lambda, \epsilon) \approx\left(\lambda_{0}, 0\right)$. This locally defined function $z_{*}$ can be extended to the entire domain stated in (5.10) by repeating this procedure for all $\lambda_{0} \in i \mathbb{R}$ and using the symmetry (2.9).

Write $\eta_{2}=\eta_{*}$ and choose $\delta_{0}>0$ and $\eta_{1}>0$ to be so small that

$$
\eta_{1} \leq \frac{1}{2} \eta_{*} \quad \text { and } \quad \delta_{0}<c(\epsilon) \eta_{1}<\delta_{0}^{*}
$$

for all $\epsilon \in\left[0, \delta_{\epsilon}\right]$. The statement now follows from Lemma 5.3 and the properties of $z_{*}$.

Proof of Proposition 5.2. Item (i) follows from Lemma 5.3. Item (ii) follows from Lemma 5.4 by noting that any $\lambda \in \mathbb{C}$ that has $-\gamma \epsilon<\lambda<\delta_{0}^{*}$ lies to the right of the essential spectrum, which in view of $z_{*}(\lambda, 0)=-\lambda / c_{*}$ means that $\operatorname{Re} z_{*}(\lambda, \epsilon)<$ 0 . Item (iii) can be explicitly computed by fixing $\nu \in \mathbb{R}$ and noting that the characteristic equation $\operatorname{det} \Delta_{\mathcal{L}(\epsilon)}(\lambda, i \nu)=0$ is a quadratic polynomial with respect to $\lambda$. 
5.2. Point spectrum. We are now ready to study the point spectrum $\sigma_{p}(\mathcal{L}(\epsilon))$. In particular, we will look for bounded solutions $(u, w)$ to the MFDE

$$
\begin{aligned}
& c(\epsilon) u^{\prime}=L(\bar{u}(\epsilon)) u-w-\lambda u, \\
& c(\epsilon) w^{\prime}=\epsilon(u-\gamma w)-\lambda w .
\end{aligned}
$$

In view of the observation (2.9), we only need to look for eigenvalues $\lambda$ in the horizontal strip $|\operatorname{Im} \lambda| \leq c(\epsilon) \pi$. Since we wish to use arguments that work uniformly for $\epsilon \in\left(0, \delta_{\epsilon}\right]$, we will decrease $\delta_{\epsilon}$ to ensure that $c(\epsilon)<\frac{3}{2} c_{*}<2 c(\epsilon)$ holds for all $\epsilon \in\left[0, \delta_{\epsilon}\right]$ and use the slightly larger strip

$$
R=\left\{\lambda \in \mathbb{C} \mid-\frac{3}{2} \pi c_{*} \leq \operatorname{Im} \lambda \leq \frac{3}{2} \pi c_{*}\right\} .
$$

As illustrated in Figure 11, the strip $R$ can be split into three subregions that we will each consider separately. In particular, we choose constants $M>0, \delta_{0}>0$ and $\delta_{\lambda}>0$ and introduce the regions

$$
\begin{array}{ll}
R_{1}\left(\delta_{1}\right) & =\left\{\lambda \in R:|\lambda|<\delta_{1}\right\}, \\
R_{2}\left(\delta_{0}, \delta_{1}, M\right) & =\left\{\lambda \in R:-\delta_{0} \leq \operatorname{Re} \lambda \leq M \text { and }|\lambda| \geq \delta_{1}\right\}, \\
R_{3}(M) & =\{\lambda \in R: \operatorname{Re} \lambda>M\} .
\end{array}
$$

Note that the translational eigenvalue $\lambda=0$ is contained in $R_{1}$. We expect to find an additional eigenvalue or resonance pole in $R_{1}$, but will rule out eigenvalues in the regions $R_{2}$ and $R_{3}$.

We recall the exponent $\eta>0$ that appears in Proposition 5.2. A direct consequence of this result and [MP99a, Prop 7.2] is that for any $\lambda$ to the right of the line $\operatorname{Re} \lambda=-\gamma \epsilon$, the set of bounded solutions $(u, w)$ to (5.16) is identical to the set of solutions to this equation that behave as $(u, w)(\xi)=O\left(e^{2 \eta \xi}\right)$ as $\xi \rightarrow \pm \infty$. For any $\nu \in \mathbb{R}$ and any function $f$, we will use the notation $e_{\nu} f$ to denote the exponentially shifted function

$$
\left[e_{\nu} f\right](\xi)=e^{\nu \xi} f(\xi)
$$

Inserting the Ansatz

$$
(u, w)=e_{2 \eta}(\widetilde{u}, \widetilde{w})
$$

into (5.16), we see that $(\widetilde{u}, \widetilde{w})$ must satisfy the new equation

$$
\begin{aligned}
c(\epsilon)(D+2 \eta) u & =L_{2 \eta}(\bar{u}(\epsilon)) u-w-\lambda u, \\
c(\epsilon)(D+2 \eta) w & =\epsilon(u-\gamma w)-\lambda w .
\end{aligned}
$$

Here we have dropped the tildes and introduced the notation

$$
\left[L_{2 \eta}(\bar{u}) v\right](\xi)=\alpha\left[e^{2 \eta} v(\xi+1)+e^{-2 \eta} v(\xi-1)-2 v(\xi)\right]+g^{\prime}(\bar{u}(\xi)) v(\xi) .
$$

We will write $\mathcal{L}_{2 \eta}(\epsilon): B C_{0}^{1}(\mathbb{R}, \mathbb{C}) \rightarrow B C_{0}(\mathbb{R}, \mathbb{C})$ for the exponentially shifted version of $\mathcal{L}(\epsilon)$ which acts as

$$
\mathcal{L}_{2 \eta}(\epsilon)(u, w)=-c(\epsilon)(D+2 \eta)(u, w)+\left(L_{2 \eta}(\bar{u}(\epsilon)) u-w, \epsilon(u-\gamma w)\right) .
$$

Throughout the remainder of this section, we will look for bounded solutions to (5.21) instead of (5.16). The advantage of this approach is that the essential spectrum has been shifted away from the imaginary axis, uniformly for $\epsilon \in\left[0, \delta_{\epsilon}\right]$ and $\operatorname{Re} \lambda \geq-\delta_{0}$. This will allow us to look for potential eigenvalues in the regions $R_{1}$ and $R_{2}$ without the need to worry about the location of $\lambda$ relative to the essential spectrum. 
Region $R_{1}$. In order to locate potential eigenvalues in the region $R_{1}$, we will use a two-stepped procedure that mimics the construction of the travelling pulses $(\bar{u}, \bar{w})(\epsilon)$ as outlined in $\varangle$. Indeed, we will first consider the MFDE

$$
\begin{aligned}
c(\epsilon)(D+2 \eta) u & =L_{2 \eta}\left(\mathcal{A}_{\mathrm{qf}}\left(\epsilon, \xi_{0}\right)\right) u-w-\lambda u, \\
c(\epsilon)(D+2 \eta) w & =\epsilon(u-\gamma w)-\lambda w,
\end{aligned}
$$

in which the function $\mathcal{A}_{\mathrm{qf}}$, which is related to the quasi-fronts described in Proposition 4.2 is given by

$$
\mathcal{A}_{\mathrm{qf}}\left(\epsilon, \xi_{0}\right)(\xi)= \begin{cases}\bar{u}_{\mathrm{qf}}^{-}\left(\epsilon, \xi_{0}\right)(\xi), & \xi \leq 0, \\ \bar{u}_{\mathrm{qf}}^{+}\left(\epsilon, \xi_{0}\right)(\xi), & \xi>0 .\end{cases}
$$

In addition, we will be interested in the counterpart of (5.24) associated to the quasi-backs, which is given by

$$
\begin{aligned}
c(\epsilon)(D+2 \eta) u & =L_{2 \eta}\left(\mathcal{A}_{\mathrm{qb}}\left(\epsilon, \xi_{0}\right)\right) u-w-\lambda u, \\
c(\epsilon)(D+2 \eta) w & =\epsilon(u-\gamma w)-\lambda w,
\end{aligned}
$$

now with

$$
\mathcal{A}_{\mathrm{qb}}\left(\epsilon, \xi_{0}\right)(\xi)= \begin{cases}\bar{u}_{\mathrm{qb}}^{-}\left(\epsilon, \xi_{0}\right)(\xi), & \xi \leq 0 \\ \bar{u}_{\mathrm{qb}}^{+}\left(\epsilon, \xi_{0}\right)(\xi), & \xi>0 .\end{cases}
$$

For notational convenience, we introduce the parameter $p=\left(\xi_{0}, \epsilon\right)$ which we take from the set

$$
\mathcal{D}_{p}=\mathcal{D}_{p}\left(\xi_{*}, \delta_{\tilde{\epsilon}}\right)=\left\{\left(\xi_{0}, \epsilon\right) \mid \xi_{0} \geq \xi_{*} \text { and } 0 \leq \epsilon \xi_{0} \leq \delta_{\tilde{\epsilon}}\right\}
$$

Proposition 5.5 (See $\left\lceil 6.1\right.$ ). Pick a sufficiently large constant $\xi_{*}>0$ and sufficiently small constants $\delta_{\tilde{\epsilon}}>0$ and $\delta_{\lambda}>0$. Then for every $p \in \mathcal{D}_{p}\left(\xi_{*}, \delta_{\tilde{\epsilon}}\right)$ and any $\lambda \in \mathbb{C}$ that has $|\lambda| \leq \delta_{\lambda}$, there exists a unique set of functions

$$
\begin{aligned}
& u_{\mathrm{qf}}^{-}(p, \lambda), u_{\mathrm{qb}}^{-}(p, \lambda) \in B C_{-\eta}((-\infty, 1], \mathbb{C}), \\
& u_{\mathrm{qf}}^{+}(p, \lambda), u_{\mathrm{qb}}^{+}(p, \lambda) \in B C_{-\eta}([-1, \infty), \mathbb{C}), \\
& w_{\mathrm{qf}}(p, \lambda), w_{\mathrm{qb}}(p, \lambda) \in B C_{-\eta}(\mathbb{R}, \mathbb{C})
\end{aligned}
$$

that satisfies the following properties.

(i) The pairs $\left(u_{\mathrm{qf}}^{ \pm}, w_{\mathrm{qf}}\right)$ satisfy (5.24) on the intervals $\mathbb{R}_{ \pm}$. Similarly, the pairs $\left(u_{\mathrm{qb}}^{ \pm}, w_{\mathrm{qb}}\right)$ satisfy (5.26) on the intervals $\mathbb{R}_{ \pm}$.

(ii) For any $p \in \mathcal{D}_{p}\left(\xi_{*}, \delta_{\tilde{\epsilon}}\right)$ and $|\lambda| \leq \delta_{\lambda}$, we have

$$
\begin{aligned}
& \Pi_{B_{f}} \mathrm{ev}_{0} e_{2 \eta} u_{\mathrm{qf}}^{-}(p, \lambda)=\Pi_{B_{f}} \mathrm{ev}_{0} e_{2 \eta} u_{\mathrm{qf}}^{-}(p, \lambda)=\mathrm{ev}_{0} q_{f}^{\prime}, \\
& \Pi_{B_{b}} \mathrm{ev}_{0} e_{2 \eta} u_{\mathrm{qb}}^{-}(p, \lambda)=\Pi_{B_{b}} \mathrm{ev}_{0} e_{2 \eta} u_{\mathrm{qb}}^{-}(p, \lambda)=\mathrm{ev}_{0} q_{b}^{\prime} .
\end{aligned}
$$

(iii) For any $p \in \mathcal{D}_{p}\left(\xi_{*}, \delta_{\tilde{\epsilon}}\right)$ and $|\lambda| \leq \delta_{\lambda}$, we have

$$
\begin{aligned}
& \xi_{f}^{\infty}(p, \lambda):=\mathrm{ev}_{0} e_{2 \eta} u_{\mathrm{qf}}^{-}(p, \lambda)-\mathrm{ev}_{0} e_{2 \eta} u_{\mathrm{qf}}^{+}(p, \lambda) \in \Gamma_{f}, \\
& \xi_{b}^{\infty}(p, \lambda):=\mathrm{ev}_{0} e_{2 \eta} u_{\mathrm{qb}}^{-}(p, \lambda)-\mathrm{ev}_{0} e_{2 \eta} u_{\mathrm{qb}}^{+}(p, \lambda) \in \Gamma_{b} .
\end{aligned}
$$

In addition, for any $\xi_{0} \geq \xi_{*}$, the maps

$$
\begin{aligned}
(\widetilde{\epsilon}, \lambda) & \mapsto\left(u_{\mathrm{qf}}^{-}\left(\xi_{0}, \widetilde{\epsilon} / \xi_{0}, \lambda\right), u_{\mathrm{qf}}^{+}\left(\xi_{0}, \widetilde{\epsilon} / \xi_{0}, \lambda\right), w_{\mathrm{qf}}\left(\xi_{0}, \widetilde{\epsilon} / \xi_{0}, \lambda\right)\right), \\
(\widetilde{\epsilon}, \lambda) & \mapsto\left(u_{\mathrm{qb}}^{-}\left(\xi_{0}, \widetilde{\epsilon} / \xi_{0}, \lambda\right), u_{\mathrm{qb}}^{+}\left(\xi_{0}, \widetilde{\epsilon} / \xi_{0}, \lambda\right), w_{\mathrm{qb}}\left(\xi_{0}, \widetilde{\epsilon} / \xi_{0}, \lambda\right)\right), \\
(\widetilde{\epsilon}, \lambda) & \mapsto\left(\xi_{f}^{\infty}\left(\xi_{0}, \widetilde{\epsilon} / \xi_{0}, \lambda\right), \xi_{b}^{\infty}\left(\xi_{0}, \widetilde{\epsilon} / \xi_{0}, \lambda\right)\right)
\end{aligned}
$$


are $C^{2}$-smooth with derivatives that can be bounded independently of $\xi_{0}$, with

$$
\xi_{f}^{\infty}\left(\xi_{0}, 0,0\right)=0, \quad \xi_{b}^{\infty}\left(\xi_{0}, 0,0\right)=0 .
$$

In addition, pick the unique $d_{f} \in \mathbb{K}_{f}$ and $d_{b} \in \mathbb{K}_{b}$ that have $d_{f}(0)=1$ and $d_{b}(0)=1$. Then there exists $C>0$ such that for any $\xi_{0} \geq \xi_{*}$ we have the estimates

$$
\begin{aligned}
& \left|\left\langle\mathrm{ev}_{0} d_{f}, D_{\epsilon} \xi_{f}^{\infty}\left(\xi_{0}, 0,0\right)\right\rangle-M_{f, \epsilon}\right| \leq C \xi_{0} e^{-\eta \xi_{0}} \\
& \left|\left\langle\mathrm{ev}_{0} d_{f}, D_{\lambda} \xi_{f}^{\infty}\left(\xi_{0}, 0,0\right)\right\rangle-M_{f, \lambda}\right| \leq C e^{-\eta \xi_{0}} \\
& \left|\left\langle\mathrm{ev}_{0} d_{b}, D_{\epsilon} \xi_{b}^{\infty}\left(\xi_{0}, 0,0\right)\right\rangle-M_{b, \epsilon}\right| \leq C \xi_{0} e^{-\eta \xi_{0}} \\
& \left|\left\langle\mathrm{ev}_{0} d_{b}, D_{\lambda} \xi_{b}^{\infty}\left(\xi_{0}, 0,0\right)\right\rangle-M_{b, \lambda}\right| \leq C e^{-\eta \xi_{0}}
\end{aligned}
$$

with

$$
\begin{aligned}
& M_{f, \epsilon}=0, \\
& M_{f, \lambda}=-\int_{-\infty}^{\infty} d_{f}(\xi) q_{f}^{\prime}(\xi) d \xi<0, \\
& M_{b, \epsilon}=c_{*}^{-1}\left(s_{R}\left(w_{*}\right)-\gamma w_{*}\right) \int_{-\infty}^{\infty} d_{b}(\xi) d \xi>0, \\
& M_{b, \lambda}=-\int_{-\infty}^{\infty} d_{b}(\xi) q_{b}^{\prime}(\xi) d \xi>0 .
\end{aligned}
$$

We are now ready to turn our attention to the linearization of (4.2) near the quasi-solutions described in Proposition 4.3. Recalling the parameter $\omega=\left(\xi_{0}, T^{\mathrm{sl}}, T\right)$ that we take from the parameter space

$$
\Omega\left(\xi_{*}, \delta_{\mathrm{sl}}, M_{T}\right)=\left\{\left(\xi_{0}, T^{\mathrm{sl}}, T\right) \mid \xi_{0} \geq \xi_{*} \text { and }\left|T^{\mathrm{sl}}-T_{*}^{\mathrm{sl}}\right|<\delta_{\lambda} \text { and } T \geq M_{T} \xi_{0}\right\},
$$

we write $\epsilon=T^{\mathrm{sl}} / T$ and set out to solve the system

$$
\begin{aligned}
& c(\epsilon)(D+2 \eta) u=L_{2 \eta}\left(\mathcal{A}_{\mathrm{qs}}(\omega)\right) u-w-\lambda u, \\
& c(\epsilon)(D+2 \eta) w=\epsilon(u-\gamma w)-\lambda w,
\end{aligned}
$$

in which the function $\mathcal{A}_{\mathrm{qs}}$ is given by

$$
\mathcal{A}_{\mathrm{qs}}(\omega)(\xi)= \begin{cases}\bar{u}_{\mathrm{qsf}}(\omega)(\xi), & \xi \leq 0, \\ \bar{u}_{\mathrm{qsx}}(\omega)(\xi), & 0 \leq \xi \leq T, \\ \bar{u}_{\mathrm{qsb}}(\omega)(\xi), & \xi \geq T .\end{cases}
$$

Proposition 5.6 (See 6.2 ). Pick sufficiently large constants $\xi_{*}>0, M_{T}>0$ and sufficiently small constants $\delta_{\mathrm{sl}}>0$ and $\delta_{\lambda}>0$. Then for each $\omega=\left(\xi_{0}, T^{\mathrm{sl}}, T\right) \in$ $\Omega=\Omega\left(\xi_{*}, \delta_{\mathrm{sl}}, M_{T}\right)$ and $\lambda \in \mathbb{C}$ that has $|\lambda|<\delta_{\lambda}$, there exists a unique quadruplet $\left(u_{\mathrm{qsf}}(\omega, \lambda), u_{\mathrm{qsb}}(\omega, \lambda), u_{\mathrm{qsx}}(\omega, \lambda), w_{\mathrm{qs}}(\omega, \lambda)\right)$ with

$$
\begin{array}{ll}
u_{\mathrm{qsf}}(\omega, \lambda) & \in \mathcal{L}\left(\mathbb{C}^{2}, B C_{0}((-\infty, 1], \mathbb{C})\right), \\
u_{\mathrm{qsb}}(\omega, \lambda) & \in \mathcal{L}\left(\mathbb{C}^{2}, B C_{0}([T-1, \infty), \mathbb{C})\right), \\
u_{\mathrm{qsx}}(\omega, \lambda) & \in \mathcal{L}\left(\mathbb{C}^{2}, B C_{0}([-1, T+1], \mathbb{C})\right), \\
w_{\mathrm{qs}}(\omega, \lambda) & \in \mathcal{L}\left(\mathbb{C}^{2}, B C_{0}(\mathbb{R}, \mathbb{C})\right)
\end{array}
$$

that satisfies the following properties.

(i) The pair $\left(u_{\mathrm{qsf}}, w_{\mathrm{qs}}\right)$ satisfies (5.37) on $(-\infty, 0]$, the pair $\left(u_{\mathrm{qsx}}, w_{\mathrm{qs}}\right)$ satisfies (5.37) on $[0, T]$ and the pair $\left(u_{\mathrm{qsb}}, w_{\mathrm{qs}}\right)$ satisfies (5.37) on $[T, \infty)$. 
$(5.40)$

(ii) For any $\omega \in \Omega$ and $|\lambda| \leq \delta_{\lambda}$, we have

$$
\begin{aligned}
& \Pi_{B_{f}} \mathrm{ev}_{0} e_{2 \eta} u_{\mathrm{qsf}}(\omega, \lambda)\left(\tilde{\kappa}_{f}, \tilde{\kappa}_{b}\right)=\Pi_{B_{f}} \mathrm{ev}_{0} e_{2 \eta} u_{\mathrm{qsx}}(\omega, \lambda)\left(\tilde{\kappa}_{f}, \tilde{\kappa}_{b}\right)=\tilde{\kappa}_{f} \mathrm{ev}_{0} q_{f}^{\prime} \text {, } \\
& \Pi_{B_{b}} e_{2 \eta} \mathrm{ev}_{T} u_{\mathrm{qsx}}(\omega, \lambda)\left(\tilde{\kappa}_{f}, \tilde{\kappa}_{b}\right)=\Pi_{B_{b}} e_{2 \eta} \operatorname{ev}_{T} u_{\mathrm{qsb}}(\omega, \lambda)\left(\tilde{\kappa}_{f}, \tilde{\kappa}_{b}\right)=\tilde{\kappa}_{b} \mathrm{ev}_{0} q_{b}^{\prime} \\
& \quad \text { for all }\left(\tilde{\kappa}_{f}, \tilde{\kappa}_{b}\right) \in \mathbb{C}^{2} . \\
& \text { (iii) For any } \omega \in \Omega \text { and }|\lambda| \leq \delta_{\lambda} \text {, we have }
\end{aligned}
$$

$$
\begin{aligned}
& \xi_{f}(\omega, \lambda):=\operatorname{ev}_{0} e_{2 \eta}\left[u_{\mathrm{qsf}}(\omega)-u_{\mathrm{qsx}}(\omega)\right] \in \mathcal{L}\left(\mathbb{C}^{2}, \Gamma_{f}\right), \\
& \xi_{b}(\omega, \lambda):=\quad \operatorname{ev}_{T} e_{2 \eta}\left[u_{\mathrm{qsx}}(\omega)-u_{\mathrm{qsb}}(\omega)\right] \in \mathcal{L}\left(\mathbb{C}^{2}, \Gamma_{b}\right) .
\end{aligned}
$$

In addition, for every $\xi_{0} \geq \xi_{*}$, the maps $\xi_{f}$ and $\xi_{b}$ are $C^{1}$-smooth with respect to $T^{\mathrm{sl}}$,

$T$ and $\lambda$. Finally, consider the maps $\widetilde{\xi}_{f}(\omega, \lambda) \in \mathcal{L}\left(\mathbb{C}^{2}, \Gamma_{f}\right)$ and $\widetilde{\xi}_{b}(\omega, \lambda) \in \mathcal{L}\left(\mathbb{C}^{2}, \Gamma_{b}\right)$ defined by

$$
\begin{aligned}
& \widetilde{\xi}_{f}(\omega, \lambda)\left(\tilde{\kappa}_{f}, \tilde{\kappa}_{b}\right):=\xi_{f}(\omega, \lambda)\left(\tilde{\kappa}_{f}, \tilde{\kappa}_{b}\right)-\xi_{f}^{\infty}\left(\xi_{0}, T^{\mathrm{sl}} / T, \lambda\right) \tilde{\kappa}_{f}, \\
& \widetilde{\xi}_{b}(\omega, \lambda)\left(\tilde{\kappa}_{f}, \tilde{\kappa}_{b}\right):=\xi_{b}(\omega, \lambda)\left(\tilde{\kappa}_{f}, \tilde{\kappa}_{b}\right)-\xi_{b}^{\infty}\left(\xi_{0}, T^{\mathrm{sl}} / T, \lambda\right) \tilde{\kappa}_{b} .
\end{aligned}
$$

Then there exists a constant $C>0$ such that for every $\omega \in \Omega$ and $|\lambda|<\delta_{\lambda}$, we have the estimate

$$
\left|\widetilde{\xi}_{f}(\omega, \lambda)\right|+\left|\widetilde{\xi}_{b}(\omega, \lambda)\right|+\left|D_{\left(T^{\mathrm{sl}}, T, \lambda\right)} \widetilde{\xi}_{f}(\omega, \lambda)\right|+\left|D_{\left(T^{\mathrm{sl}}, T, \lambda\right)} \widetilde{\xi}_{b}(\omega, \lambda)\right| \leq C e^{-\eta T}
$$

In order to solve the eigenvalue problem $\mathcal{L}_{2 \eta}(\epsilon)(u, w)=\lambda(u, w)$ for $\lambda \in R_{1}$, it hence suffices to analyze the bifurcation equations

$$
\left(\begin{array}{l}
\xi_{f}\left(\omega_{\mathrm{nl}}(\epsilon), \lambda\right) \\
\xi_{b}\left(\omega_{\mathrm{nl}}(\epsilon), \lambda\right)
\end{array}\right)\left(\begin{array}{l}
\tilde{\kappa}_{f} \\
\tilde{\kappa}_{b}
\end{array}\right)=0
$$

The results obtained so far imply that there is a $\beta>0$ so that

$$
\begin{aligned}
& \left(\begin{array}{l}
\xi_{f}\left(\omega_{\mathrm{nl}}(\epsilon), \lambda\right) \\
\xi_{b}\left(\omega_{\mathrm{nl}}(\epsilon), \lambda\right)
\end{array}\right) \\
& =\left(\begin{array}{cc}
\lambda M_{f, \lambda}+O\left(\lambda^{2}+\epsilon(|\epsilon|+|\lambda|)|\ln \epsilon|\right) & O\left(e^{-\beta / \epsilon}\right) \\
O\left(e^{-\beta / \epsilon}\right) & \lambda M_{b, \lambda}+\epsilon M_{b, \epsilon}+O\left(\lambda^{2}+\epsilon(|\epsilon|+|\lambda|)|\ln \epsilon|\right)
\end{array}\right),
\end{aligned}
$$

where $M_{f, \lambda}, M_{b, \lambda}, M_{b, \epsilon}$ are given in (5.35).

Before we continue with the analysis of equation (5.44), we comment on its interpretation. The estimates in Proposition 5.6 show that the solutions to the weighted eigenvalue problem are obtained by gluing together the weighted Nagumo eigenfunctions $e^{2 \eta \xi} q_{f}^{\prime}(\xi)$ and $e^{2 \eta \xi} q_{b}^{\prime}(\xi)$ with amplitudes $\left(\tilde{\kappa}_{f}, \tilde{\kappa}_{b}\right)$. Since the first jump occurs at $\xi=0$ and the second jump at $\xi=T$ with $T \geq \delta / \epsilon$, we see that we can alternatively use the amplitudes $\kappa_{f}:=\tilde{\kappa}_{f}$ and $\kappa_{b}:=e^{2 \eta T} \tilde{\kappa}_{b}$ of the unweighted Nagumo eigenfunctions $q_{f}^{\prime}$ and $q_{b}^{\prime}$ to obtain the equivalent system

$$
\begin{gathered}
\left(\begin{array}{cc}
\lambda M_{f, \lambda}+O\left(\lambda^{2}+\epsilon(|\epsilon|+|\lambda|)|\ln \epsilon|\right) & e^{-2 \eta T} O\left(e^{-\beta / \epsilon}\right) \\
e^{2 \eta T} O\left(e^{-\beta / \epsilon}\right) & \lambda M_{b, \lambda}+\epsilon M_{b, \epsilon}+O\left(\lambda^{2}+\epsilon(|\epsilon|+|\lambda|)|\ln \epsilon|\right)
\end{array}\right) \\
\times\left(\begin{array}{c}
\kappa_{f} \\
\kappa_{b}
\end{array}\right)=0 .
\end{gathered}
$$

The next three results establish that (S2), (S3) and part of (S1) in 92 hold for $\mathcal{L}(\epsilon)$. 
Corollary 5.7. There exists $\delta_{\epsilon}>0$ such that for all $0<\epsilon<\delta_{\epsilon}$, the only solutions $(u, w) \in B C_{0}^{1}\left(\mathbb{R}, \mathbb{C}^{2}\right)$ to the equation

$$
\mathcal{L}_{2 \eta}(\epsilon)(u, w)=0
$$

are $(u, w)=e_{-2 \eta}(\bar{u}, \bar{w})(\epsilon)$ and scalar multiples thereof, while the equation

$$
\mathcal{L}_{2 \eta}(\epsilon)(u, w)=\left(e_{-2 \eta} \bar{u}^{\prime}(\epsilon), e_{-2 \eta} \bar{w}^{\prime}(\epsilon)\right)
$$

admits no solutions $(u, w) \in B C_{0}^{1}\left(\mathbb{R}, \mathbb{C}^{2}\right)$. In particular, $\lambda=0$ is a simple eigenvalue for $\mathcal{L}_{2 \eta}(\epsilon)$ and hence also for $\mathcal{L}(\epsilon)$.

Proof. The fact that $M_{f, \epsilon}=0$ and $M_{b, \epsilon} \neq 0$ implies that

$$
\operatorname{dim} \operatorname{Ker}\left(\begin{array}{c}
\xi_{f}\left(\omega_{\mathrm{nl}}(\epsilon), 0\right) \\
\xi_{b}\left(\omega_{\mathrm{nl}}(\epsilon), 0\right)
\end{array}\right) \leq 1
$$

for sufficiently small $\epsilon>0$. Since

$$
\mathcal{L}_{2 \eta}(\epsilon)\left(e_{-2 \eta} \bar{u}^{\prime}(\epsilon), e_{-2 \eta} \bar{w}^{\prime}(\epsilon)\right)=0
$$

holds by construction, the statement concerning (5.46) follows.

In view of (5.49), there exists a pair $\tilde{\kappa}_{f}(\epsilon)$ and $\tilde{\kappa}_{b}(\epsilon)$ for which

$$
e_{-2 \eta}\left(\bar{u}^{\prime}(\epsilon), \bar{w}^{\prime}(\epsilon)\right) \sim\left(u_{\mathrm{qsf}}, u_{\mathrm{qsx}}, u_{\mathrm{qsb}}, w_{\mathrm{qs}}\right)\left(\omega_{\mathrm{nl}}(\epsilon), 0\right)\left(\tilde{\kappa}_{f}(\epsilon), \tilde{\kappa}_{b}(\epsilon)\right) .
$$

Here the symbol $\sim$ means that the function on the left can be obtained by appropriately concatenating the functions on the right. Differentiating (5.37) with respect to $\lambda$ and evaluating at $\omega=\omega_{\mathrm{nl}}(\epsilon)$ and $\lambda=0$, we find that the only possible candidate for a solution to (5.47) is given by

$$
(u, w) \sim D_{\lambda}\left(u_{\mathrm{qsf}}, u_{\mathrm{qsx}}, u_{\mathrm{qsb}}, w_{\mathrm{qs}}\right)\left(\omega_{\mathrm{nl}}(\epsilon), 0\right)\left(\tilde{\kappa}_{f}(\epsilon), \tilde{\kappa}_{b}(\epsilon)\right),
$$

which is an actual solution if and only if

$$
D_{\lambda} \xi_{f}\left(\omega_{\mathrm{nl}}(\epsilon), 0\right)\left(\tilde{\kappa}_{f}(\epsilon), \tilde{\kappa}_{b}(\epsilon)\right)=D_{\lambda} \xi_{b}\left(\omega_{\mathrm{nl}}(\epsilon), 0\right)\left(\tilde{\kappa}_{f}(\epsilon), \tilde{\kappa}_{b}(\epsilon)\right)=0 .
$$

However, for all sufficiently small $\epsilon>0$ this is precluded by the Melnikov identities (5.35).

Corollary 5.8. Pick any sufficiently small $\delta_{\lambda}>0$. Then there exists $\delta_{\epsilon}>0$ such that the following holds true. For every $0<\epsilon<\delta_{\epsilon}$, there exists a unique $\lambda_{2}=\lambda_{2}(\epsilon) \in \mathbb{C} \backslash\{0\}$ that has $\left|\lambda_{2}\right|<\delta_{\lambda}$ and

$$
\operatorname{dim} \operatorname{Ker}\left(\mathcal{L}_{2 \eta}(\epsilon)-\lambda_{2}\right)>0 .
$$

In addition, $\lambda_{2}$ is real and admits the expansion

$$
\lambda_{2}(\epsilon)=-\epsilon M_{b, \lambda}^{-1} M_{b, \epsilon}+O\left(\epsilon^{2} \ln (1 / \epsilon)\right) .
$$

In particular, if

$$
M_{b, \lambda}^{-1} M_{b, \epsilon}<\gamma+a^{-1},
$$

then $\lambda_{2}(\epsilon)$ is an eigenvalue for the unshifted operator $\mathcal{L}(\epsilon)$.

Proof. Consider any small $\epsilon>0$ and suppose that (5.53) holds for some small $\lambda \in \mathbb{C}$. This implies that (5.44) holds for some nonzero pair $\left(\tilde{\kappa}_{f}, \tilde{\kappa}_{b}\right) \in \mathbb{C}^{2}$. Writing

$$
\begin{aligned}
& \xi_{f}^{\infty}\left(\xi_{0}, \epsilon, \lambda\right)=\lambda \widetilde{M}_{f, \lambda}\left(\xi_{0}, \epsilon, \lambda\right)+\epsilon \widetilde{M}_{f, \epsilon}\left(\xi_{0}, \epsilon, \lambda\right), \\
& \xi_{b}^{\infty}\left(\xi_{0}, \epsilon, \lambda\right)=\lambda \widetilde{M}_{b, \lambda}\left(\xi_{0}, \epsilon, \lambda\right)+\epsilon \widetilde{M}_{b, \epsilon}\left(\xi_{0}, \epsilon, \lambda\right),
\end{aligned}
$$


and also

$$
\begin{aligned}
\widetilde{M}_{f}\left(\xi_{0}, \lambda, \epsilon\right) & =\widetilde{M}_{f, \lambda}\left(\xi_{0}, \lambda, \epsilon\right)^{-1} \widetilde{M}_{f, \epsilon}\left(\xi_{0}, \lambda, \epsilon\right), \\
\widetilde{M}_{b}\left(\xi_{0}, \lambda, \epsilon\right) & =\widetilde{M}_{b, \lambda}\left(\xi_{0}, \lambda, \epsilon\right)^{-1} \widetilde{M}_{b, \epsilon}\left(\xi_{0}, \lambda, \epsilon\right), \\
M_{b} & =M_{b, \lambda}^{-1} M_{b, \epsilon},
\end{aligned}
$$

we find the bifurcation equation

$$
\left(\lambda+\epsilon \widetilde{M}_{f}\left(\xi_{0}, \lambda, \epsilon\right)\right)\left(\lambda+\epsilon \widetilde{M}_{b}\left(\xi_{0}, \lambda, \epsilon\right)\right)+h\left(\xi_{0}, \lambda, \epsilon\right)=0 .
$$

Here $h\left(\xi_{0}, \cdot\right), \widetilde{M}_{f}\left(\xi_{0}, \cdot\right)$ and $\widetilde{M}_{b}\left(\xi_{0}, \cdot\right)$ are $C^{1}$-smooth, with

$$
\begin{aligned}
|h|+\left|D_{(\lambda, \epsilon)} h\right| & \leq C e^{-\beta / \epsilon}, \\
\left|\widetilde{M}_{f}\right|+\left|\widetilde{M}_{b}-M_{b}\right| & \leq C\left(|\lambda|+\xi_{0} \epsilon+\xi_{0} e^{-\eta \xi_{0}}\right), \\
\left|D_{(\lambda, \tilde{\epsilon})} \widetilde{M}_{f}\right|+\left|D_{(\lambda, \tilde{\epsilon})} \widetilde{M}_{b}\right| & \leq C,
\end{aligned}
$$

for some constants $C>0$ and $\beta>0$, in which $\tilde{\epsilon}=\epsilon / \xi_{0}$. Continuity in $\epsilon$ and the argument principle show that for every sufficiently small $\delta_{\lambda}>0$, we may find $\delta_{\tilde{\epsilon}}>0$ such that (5.58) admits precisely two roots $\lambda$ that have $|\lambda|<\delta_{\lambda}$ for all $0<\epsilon \xi_{0}<\delta_{\tilde{\epsilon}}$.

A priori we know that one of these roots must be zero. Let us therefore treat the bifurcation equation as a second-degree polynomial in $\lambda$ and look for the second root $\lambda_{2}$. Writing

$$
z=\lambda_{2}+\epsilon M_{b},
$$

we find that $z$ must solve the fixed point equation

$$
z=\mathcal{F}\left(\xi_{0}, \epsilon, z-\epsilon M_{b}\right)
$$

in which

$$
\begin{aligned}
\mathcal{F}\left(\xi_{0}, \epsilon, \lambda\right)= & -\frac{1}{2} \epsilon\left(\widetilde{M}_{f}+\widetilde{M}_{b}-M_{b}\right) \\
& +\frac{1}{2} \epsilon \frac{\left(M_{b}-\widetilde{M}_{2}\right)\left(M_{b}+\widetilde{M}_{2}\right)+\widetilde{M}_{f}\left(2 \widetilde{M}_{b}-\widetilde{M}_{f}\right)+4 \epsilon^{-2} h}{M_{b}+\sqrt{\left(\widetilde{M}_{1}-\widetilde{M}_{2}\right)^{2}-4 \epsilon^{-2} h}} .
\end{aligned}
$$

There exists $C>0$ such that for all $\lambda$ with $|\lambda| \leq 2 \epsilon M_{b}$, we have for all $\xi_{0} \geq \xi_{*}$,

$$
\left|\mathcal{F}\left(\xi_{0}, \epsilon, \lambda\right)\right| \leq C \epsilon\left(\xi_{0} \epsilon+\xi_{0} e^{-\eta \xi_{0}}\right) .
$$

Choosing $e^{-\eta \xi_{0}}=\epsilon$, we may hence use a fixed point argument to find a unique solution to (5.61) in the set $|z| \leq 2 C \epsilon \xi_{0} e^{-\eta \xi_{0}}$.

Region $R_{2}$. We pick $\delta_{0}>0$ to be smaller than the values mentioned in Lemma 3.3 and Proposition 5.2 and fix this constant throughout the rest of this section. We will show that no eigenvalues can exist in $R_{2}$, which is a consequence of the splitting

$$
C([-1,1], \mathbb{C})=Q_{f, \lambda} \oplus P_{f, \lambda}=Q_{b, \lambda} \oplus Q_{b, \lambda}
$$

that was described in Lemma 3.3. Indeed, this splitting eliminates the freedom we had in region $R_{1}$ that allowed us to freely specify the value of the projections $\Pi_{B_{f}}$ ev $v_{0}$ and $\Pi_{B_{b}} \mathrm{ev}_{T}$ applied to the potential eigenfunctions. Since we no longer 
expect eigenvalues to exist, the estimates that we require are not as detailed as those used in our analysis of $R_{1}$. This allows us to look directly at (5.37).

Proposition 5.9 (See 6.3 . Consider any $\lambda_{0} \in \mathbb{C} \backslash\left\{2 \pi i c_{*} \mathbb{Z}\right\}$ that has $\operatorname{Re} \lambda_{0} \geq-\delta_{0}$. Then there exist large constants $\xi_{*}>0$ and $M_{T}>0$, together with small constants $\delta_{\mathrm{sl}}>0$ and $\delta_{\lambda}>0$, such that the differential equation (5.37) with $\left|\lambda-\lambda_{0}\right|<\delta_{\lambda}$ and $\omega \in \Omega\left(\xi_{*}, \delta_{\mathrm{sl}}, M_{T}\right)$ has no nontrivial solutions $(u, w) \in B C_{0}\left(\mathbb{R}, \mathbb{C}^{2}\right)$.

Corollary 5.10. For any $\delta_{1}>0$ and any $M>0$, there exist large constants $\xi_{*}>0, M_{T}>0$ and a small constant $\delta_{\mathrm{sl}}>0$ such that (5.37) admits no nontrivial solutions for all $\omega \in \Omega$ and $\lambda \in R_{2}\left(\delta_{0}, \delta_{1}, M\right)$.

Proof. This follows directly from the compactness of $R_{2}$.

Region $R_{3}$. If $\operatorname{Re} \lambda$ is sufficiently large, the nonlocal terms in (5.21) can be treated as small perturbations to the ODE

$$
c(\epsilon)(D+2 \eta)(u, w)=-\lambda(u, w) .
$$

We can hence use ODE techniques to obtain the following result.

Lemma 5.11. There exist constants $M>0$ and $\delta_{\epsilon}>0$ such that the differential equation (5.21) with $0<\epsilon<\delta_{\epsilon}$ and $\lambda \in R_{3}(M)$ has no nontrivial solutions $(u, w) \epsilon$ $B C_{0}\left(\mathbb{R}, \mathbb{C}^{2}\right)$.

Proof. Introducing the new variable $\tau=c(\epsilon)^{-1}[\operatorname{Re} \lambda] \xi$, (5.21) can be written as

$$
\left(u_{\tau}, w_{\tau}\right)=-(u, w)+[\operatorname{Re} \lambda]^{-1} \mathcal{B}(\lambda, \epsilon)(u, w) .
$$

The linear operator

$$
\mathcal{B}(\lambda, \epsilon) \in \mathcal{L}\left(B C_{0}\left(\mathbb{R}, \mathbb{C}^{2}\right)\right)
$$

can be bounded uniformly for $\epsilon \in\left(0, \delta_{\epsilon}\right]$ and $\lambda \in R$ with $\operatorname{Re} \lambda \geq 0$. It is not hard to see that for any $h \in B C_{0}\left(\mathbb{R}, \mathbb{C}^{2}\right)$, the ODE

$$
\left(u_{\tau}, w_{\tau}\right)=-(u, w)+h
$$

has a unique solution in $B C_{0}\left(\mathbb{R}, \mathbb{C}^{2}\right)$ that we will denote by $(u, w)=L_{*} h$. Solving (5.21) is now equivalent to solving

$$
(u, w)=L_{*}[\operatorname{Re} \lambda]^{-1} \mathcal{B}(\lambda, \epsilon)(u, w),
$$

which cannot have a nontrivial solution if $\operatorname{Re} \lambda$ is sufficiently large.

Proof of Proposition 5.1. Observe that the constant $\delta_{0}$ appearing in (S1) may depend on $\epsilon>0$. This allows us to establish (S1) by combining Proposition [5.2, Corollaries 5.8 and 5.10 and Lemma 5.11. Properties (S2) and (S3) follow from Corollary 5.7

\section{Proof of Propositions 5.5, 5.6 and 5.9}

In this section we provide the proofs of Propositions 5.5, 5.6] and 5.9. The techniques we use are very similar to those employed in [HS10, §5-6] and we in fact refer to those sections for many of the details. This is possible because the 
exponential shift $e_{2 \eta}$ that we applied in (5.21) does not change the structure of the spaces $P_{f, \lambda}, Q_{f, \lambda}, \widehat{P}_{f}$ and $\widehat{Q}_{f}$ discussed in 93 that are related to the first component of (5.21). Indeed, the corresponding characteristic equation (3.8) does not have roots in the strip $|\operatorname{Re} z| \leq 4 \eta$.

6.1. Region $R_{1}$ : Quasi-fronts and quasi-backs. We set out to prove Proposition 5.5. We recall the constants $\xi_{*}$ and $\delta_{\tilde{\epsilon}}$ appearing in Proposition 4.2, and pick $p=\left(\xi_{0}, \epsilon\right) \in \mathcal{D}_{p}\left(\xi_{*}, \delta_{\tilde{\epsilon}}\right)$. For the front, we split the real line into the three intervals $(-\infty, 0],\left[0, \xi_{0}\right]$ and $\left[\xi_{0}, \infty\right)$ that we each consider separately. To aid us in this scheme, we introduce the families of function spaces

(6.1)

$$
\begin{aligned}
& B C_{\mathrm{qf}, \beta}^{\ominus}=\{(v, \theta) \in C((-\infty, 1], \mathbb{C}) \times C((-\infty, 0], \mathbb{C}) \text { for which } \\
& \left.\|(v, \theta)\|_{B C_{\mathrm{q}, \beta}^{\ominus}}^{\ominus}:=\sup _{\xi \leq 1} \mathrm{e}^{-\beta|\xi|}|v(\xi)|+\sup _{\xi \leq 0} \mathrm{e}^{-\beta|\xi|}|\theta(\xi)|<\infty\right\}, \\
& B C_{\mathrm{qf}}^{\odot} \quad=\left\{(v, \theta) \in C\left(\left[-1, \xi_{0}+1\right], \mathbb{C}\right) \times C\left(\left[0, \xi_{0}\right], \mathbb{C}\right)\right. \text { for which } \\
& \left.\|(v, \theta)\|_{B C_{\mathrm{qf}}^{\odot}}^{\odot}:=\sup _{-1 \leq \xi \leq \xi_{0}+1}|v(\xi)|+\sup _{0 \leq \xi \leq \xi_{0}}|\theta(\xi)|<\infty\right\}, \\
& B C_{\mathrm{qf}, \beta}^{\oplus}=\left\{(v, \theta) \in C\left(\left[\xi_{0}-1, \infty\right), \mathbb{C}\right) \times C\left(\left[\xi_{0}, \infty\right), \mathbb{C}\right)\right. \text { for which } \\
& \|(v, \theta)\|_{B C_{\mathrm{qf}, \beta}^{\oplus}}^{\oplus}:=\sup _{\xi \geq \xi_{0}-1} \mathrm{e}^{-\beta\left|\xi-\xi_{0}\right|}|v(\xi)| \\
& \left.+\sup _{\xi \geq \xi_{0}} e^{-\beta\left|\xi-\xi_{0}\right|}|\theta(\xi)|<\infty\right\}
\end{aligned}
$$

parametrized by $\beta>0$, together with the families

$$
\begin{aligned}
B C_{\mathrm{qf}, \beta}^{-}= & \left\{\left(g_{1}, g_{2}\right) \in C((-\infty, 0], \mathbb{C}) \times C((-\infty, 0], \mathbb{C})\right. \text { for which } \\
& \left.\left\|\left(g_{1}, g_{2}\right)\right\|_{B C_{\mathrm{qf}, \beta}^{-}}:=\sup _{\xi \leq 0} \mathrm{e}^{-\beta|\xi|}\left(\left|g_{1}(\xi)\right|+\left|g_{2}(\xi)\right|\right)<\infty\right\}, \\
B C_{\mathrm{qf}}^{\diamond}= & \left\{\left(g_{1}, g_{2}\right) \in C\left(\left[0, \xi_{0}\right], \mathbb{C}\right) \times C\left(\left[0, \xi_{0}\right], \mathbb{C}\right)\right. \text { for which } \\
& \left.\left\|\left(g_{1}, g_{2}\right)\right\|_{B C_{\mathrm{qf}}^{\diamond}}:=\sup _{0 \leq \xi \leq \xi_{0}}\left|g_{1}(\xi)\right|+\left|g_{2}(\xi)\right|<\infty\right\}, \\
B C_{\mathrm{qf}, \beta}^{+}= & \left\{\left(g_{1}, g_{2}\right) \in C\left(\left[\xi_{0}, \infty\right), \mathbb{C}\right) \times C\left(\left[\xi_{0}, \infty\right), \mathbb{C}\right)\right. \text { for which } \\
& \left.\left\|\left(g_{1}, g_{2}\right)\right\|_{B C_{\mathrm{qf}, \beta}^{+}}:=\sup _{\xi \geq \xi_{0}} \mathrm{e}^{-\beta\left|\xi-\xi_{0}\right|}\left(\left|g_{1}(\xi)\right|+\left|g_{2}(\xi)\right|\right)<\infty\right\} .
\end{aligned}
$$

Notice that in contrast to the definition [HS10, Eq. (5.49)] for $B C_{\mathrm{qf}}^{\diamond}$, there is no factor $\xi_{0}$ in front of the term $\left|g_{2}(\xi)\right|$. For the backs, we use the spaces (6.3)

$$
\begin{aligned}
& B C_{\mathrm{qb}, \beta}^{\ominus}=\left\{(v, \theta) \in C\left(\left(-\infty,-\xi_{0}+1\right], \mathbb{C}\right) \times C\left(\left(-\infty,-\xi_{0}\right], \mathbb{C}\right)\right. \text { for which } \\
& \|(v, \theta)\|_{B C_{\mathrm{qf}, \beta}^{\ominus}}^{\ominus}:=\sup _{\xi \leq-\xi_{0}+1} \mathrm{e}^{-\beta\left|\xi+\xi_{0}\right|}|v(\xi)| \\
& \left.+\sup _{\xi \leq-\xi_{0}} e^{-\beta\left|\xi+\xi_{0}\right|}|\theta(\xi)|<\infty\right\}, \\
& B C_{\mathrm{qb}}^{\odot-}=\left\{(v, \theta) \in C\left(\left[-\xi_{0}-1,1\right], \mathbb{C}\right) \times C\left(\left[-\xi_{0}, 0\right], \mathbb{C}\right)\right. \text { for which } \\
& \left.\|(v, \theta)\|_{B C_{\mathrm{q}}^{\odot-}}^{\odot}:=\sup _{-\xi_{0}-1 \leq \xi \leq 1}|v(\xi)|+\sup _{-\xi_{0} \leq \xi \leq 0}|\theta(\xi)|<\infty\right\}, \\
& B C_{\mathrm{qb}}^{\odot+}=\left\{(v, \theta) \in C\left(\left[-1, \xi_{0}+1\right], \mathbb{C}\right) \times C\left(\left[0, \xi_{0}\right], \mathbb{C}\right)\right. \text { for which } \\
& \left.\|(v, \theta)\|_{B C_{\mathrm{qf}}^{\odot+}}^{\odot=}:=\sup _{-1 \leq \xi \leq \xi_{0}+1}|v(\xi)|+\sup _{0 \leq \xi \leq \xi_{0}}|\theta(\xi)|<\infty\right\}, \\
& B C_{\mathrm{qb}, \beta}^{\oplus}=\left\{(v, \theta) \in C\left(\left[\xi_{0}-1, \infty\right), \mathbb{C}\right) \times C\left(\left[\xi_{0}, \infty\right), \mathbb{C}\right)\right. \text { for which } \\
& \|(v, \theta)\|_{B C_{\mathrm{q}, \beta}^{\oplus}}:=\sup _{\xi \geq \xi_{0}-1} \mathrm{e}^{-\beta\left|\xi-\xi_{0}\right|}|v(\xi)| \\
& \left.+\sup _{\xi \geq \xi_{0}} e^{-\beta\left|\xi-\xi_{0}\right|}|\theta(\xi)|<\infty\right\}
\end{aligned}
$$


parametrized by $\beta>0$, together with the family

(6.4)

$$
\begin{aligned}
& B C_{\mathrm{qb}, \beta}^{-}=\left\{\left(g_{1}, g_{2}\right) \in C\left(\left(-\infty,-\xi_{0}\right], \mathbb{C}\right) \times C\left(\left(-\infty,-\xi_{0}\right], \mathbb{C}\right)\right. \text { for which } \\
& \left.\left\|\left(g_{1}, g_{2}\right)\right\|_{B C_{\mathrm{qb}, \beta}^{-}}:=\sup _{\xi \leq 0} \mathrm{e}^{-\beta\left|\xi+\xi_{0}\right|}\left(\left|g_{1}(\xi)\right|+\left|g_{2}(\xi)\right|\right)<\infty\right\}, \\
& B C_{\mathrm{qb}}^{\diamond-}=\left\{\left(g_{1}, g_{2}\right) \in C\left(\left[-\xi_{0}, 0\right], \mathbb{C}\right) \times C\left(\left[-\xi_{0}, 0\right], \mathbb{C}\right)\right. \text { for which } \\
& \left.\left\|\left(g_{1}, g_{2}\right)\right\|_{B C_{\mathrm{qb}}^{\diamond-}}:=\sup _{-\xi_{0} \leq \xi \leq 0}\left|g_{1}(\xi)\right|+\left|g_{2}(\xi)\right|<\infty\right\}, \\
& B C_{\mathrm{qb}}^{\diamond+}=\left\{\left(g_{1}, g_{2}\right) \in C\left(\left[0, \xi_{0}\right], \mathbb{C}\right) \times C\left(\left[0, \xi_{0}\right], \mathbb{C}\right)\right. \text { for which } \\
& \left.\left\|\left(g_{1}, g_{2}\right)\right\|_{B C_{\mathrm{ab}}^{\diamond+}}:=\sup _{0 \leq \xi \leq \xi_{0}}\left|g_{1}(\xi)\right|+\left|g_{2}(\xi)\right|<\infty\right\}, \\
& B C_{\mathrm{qb}, \beta}^{+}=\left\{\left(g_{1}, g_{2}\right) \in C\left(\left[\xi_{0}, \infty\right), \mathbb{C}\right) \times C\left(\left[\xi_{0}, \infty\right), \mathbb{C}\right)\right. \text { for which } \\
& \left.\left\|\left(g_{1}, g_{2}\right)\right\|_{B C_{\mathrm{qb}, \beta}^{+}}:=\sup _{\xi \geq \xi_{0}} \mathrm{e}^{-\beta\left|\xi-\xi_{0}\right|}\left(\left|g_{1}(\xi)\right|+\left|g_{2}(\xi)\right|\right)<\infty\right\} .
\end{aligned}
$$

We recall the constant $\eta>0$ from Proposition 5.2 and introduce the combined spaces

$$
\begin{aligned}
& \mathcal{H}_{\mathrm{qf}, \zeta}^{\circ}=B C_{\mathrm{qf},-\eta}^{\ominus} \times B C_{\mathrm{qf}}^{\odot} \times B C_{\mathrm{qf},-\eta+\zeta}^{\oplus}, \\
& \mathcal{H}_{\mathrm{qb}, \zeta}^{\odot}=B C_{\mathrm{qb},-\eta+\zeta}^{\ominus} \times B C_{\mathrm{qb}}^{\odot-} \times B C_{\mathrm{qb}}^{\odot+} \times B C_{\mathrm{qb},-\eta+\zeta}^{\oplus},
\end{aligned}
$$

parametrized by $\zeta \geq 0$, together with

$$
\begin{aligned}
& \mathcal{H}_{\mathrm{qf}}=B C_{\mathrm{qf},-\eta}^{-} \times B C_{\mathrm{qf}}^{\diamond} \times B C_{\mathrm{qf},-\eta+\zeta}^{+}, \\
& \mathcal{H}_{\mathrm{qb}}=B C_{-\eta+\zeta}^{-} \times B C^{\diamond-} \times B C^{\diamond+} \times B C_{-\eta+\zeta}^{+} .
\end{aligned}
$$

We employ the shorthands $\mathcal{H}_{\mathrm{qf}}^{\circ}=\mathcal{H}_{\mathrm{qf}, 0}^{\circ}$ and $\mathcal{H}_{\mathrm{qb}}^{\circ}=\mathcal{H}_{\mathrm{qb}, 0}^{\circ}$.

Our goal is to find sets

$$
\begin{aligned}
& h_{\mathrm{qf}}=\left(v_{\mathrm{qf}}^{-}, \theta_{\mathrm{qf}}^{-}, v_{\mathrm{qf}}^{\diamond}, \theta_{\mathrm{qf}}^{\diamond}, v_{\mathrm{qf}}^{+}, \theta_{\mathrm{qf}}^{+}\right) \in \mathcal{H}_{\mathrm{qf}}^{\circ}, \\
& h_{\mathrm{qb}}=\left(v_{\mathrm{qb}}^{-}, \theta_{\mathrm{qb}}^{-}, v_{\mathrm{qb}}^{\diamond-}, \theta_{\mathrm{qb}}^{\diamond-}, v_{\mathrm{qb}}^{\diamond+}, \theta_{\mathrm{qb}}^{\diamond+}, v_{\mathrm{qb}}^{+}, \theta_{\mathrm{qb}}^{+}\right) \in \mathcal{H}_{\mathrm{qb}}^{\circ},
\end{aligned}
$$

such that the choices

$$
\begin{gathered}
w_{\mathrm{qf}}(\xi)= \begin{cases}\theta_{\mathrm{qf}}^{-}(\xi) & \text { for } \xi \leq 0, \\
\theta_{\mathrm{qf}}^{\diamond}(\xi) & \text { for } 0 \leq \xi \leq \xi_{0}, \\
\theta_{\mathrm{qf}}^{+}(\xi) & \text { for } \xi \geq \xi_{0},\end{cases} \\
w_{\mathrm{qb}}(\xi)= \begin{cases}\theta_{\mathrm{qb}}^{-}(\xi) & \text { for } \xi \leq-\xi_{0}, \\
\theta_{\mathrm{db}}^{\diamond-}(\xi) & \text { for }-\xi_{0} \leq \xi \leq 0, \\
\theta_{\mathrm{qb}}^{\diamond}(\xi) & \text { for } 0 \leq \xi \leq \xi_{0}, \\
\theta_{\mathrm{qb}}^{+}(\xi) & \text { for } \xi \geq \xi_{0},\end{cases}
\end{gathered}
$$

in combination with

$$
\begin{aligned}
& u_{\mathrm{qf}}^{-}(\xi)=v_{\mathrm{qf}}^{-}(\xi), \\
& u_{\mathrm{qf}}^{+}(\xi)= \begin{cases}v_{\mathrm{qf}}^{\diamond}(\xi) & \text { for }-1 \leq \xi \leq \xi_{0}, \\
v_{\mathrm{qf}}^{+}(\xi) & \text { for } \xi \geq \xi_{0},\end{cases} \\
& u_{\mathrm{qb}}^{-}(\xi)=\left\{\begin{array}{cc}
v_{\mathrm{qb}}^{\diamond-}(\xi) & \text { for } \xi \leq-\xi_{0}, \\
v_{\mathrm{qb}}^{\diamond+}(\xi) & \text { for } \xi_{0} \leq \xi \leq 1,
\end{array}\right. \\
& u_{\mathrm{qb}}^{+}(\xi)= \begin{cases}v_{\mathrm{qb}}^{\diamond+}(\xi) & \text { for }-1 \leq \xi \leq \xi_{0}, \\
v_{\mathrm{qb}}^{+}(\xi) & \text { for } \xi \geq \xi_{0},\end{cases}
\end{aligned}
$$

satisfy the conditions of Proposition 5.5 . 
Inserting the above Ansatz for $\left(u_{\mathrm{qf}}^{ \pm}, w_{\mathrm{qf}}\right)$ into (5.24), we find that we must solve the linear equation

(6.10)

$\Lambda_{\mathrm{qf}}^{\#}(p)\left(v_{\mathrm{qf}}^{\#}, \theta_{\mathrm{qf}}^{\#}\right):=\left(\Lambda_{\mathrm{qf}, 1}^{\#}(p) v_{\mathrm{qf}}^{\#}, c(\epsilon)(D+2 \eta) \theta_{\mathrm{qf}}^{\#}\right)=\left(-\theta_{\mathrm{qf}}^{\#}, 0\right)+B_{\mathrm{qf}}^{\#}(p, \lambda)\left(v_{\mathrm{qf}}^{\#}, \theta_{\mathrm{qf}}^{\#}\right)$

for $\#=-, \diamond,+$. Here the differential operators are given by

$$
\begin{array}{ll}
\Lambda_{\mathrm{qf}, 1}^{\#}(p) v & =c_{*}(D+2 \eta) v-L_{2 \eta}\left(q_{f}\right) v,
\end{array} \quad \text { for } \#=-, \diamond
$$

while the inhomogeneities can be written as

$$
\begin{array}{rlrl}
B_{\mathrm{qf}, 1}^{\#}(p, \lambda)(v, \theta)= & -\lambda v+\left[g^{\prime}\left(\bar{u}_{\mathrm{qf}}(p)\right)-g^{\prime}\left(q_{f}\right)\right] v & \\
& +\frac{c_{*}-c(\epsilon)}{c(\epsilon)}\left[L_{2 \eta}\left(\bar{u}_{\mathrm{qf}}(p)\right) v-\theta-\lambda v\right], & & \text { for } \#=-, \diamond, \\
B_{\mathrm{qf}, 1}^{+}(p, \lambda)(v, \theta)= & -\lambda v+\left[g^{\prime}\left(\bar{u}_{\mathrm{qf}}(p)\right)-g^{\prime}\left(s_{R}\left(\bar{w}_{\mathrm{qf}}(p), \epsilon\right)\right)\right] v & \\
B_{\mathrm{qf}, 2}^{\#}(p, \lambda)(v, \theta)= & \epsilon(v-\gamma \theta)-\lambda \theta, & \text { for } \#=-, \diamond,+.
\end{array}
$$

Similarly, we represent the linear system that arises by inserting the Ansatz (6.8) (6.9) for $\left(u_{\mathrm{qb}}^{ \pm}, w_{\mathrm{qb}}\right)$ into (5.26) as

$\Lambda_{\mathrm{qb}}^{\#}(p)\left(v_{\mathrm{qb}}^{\#}, \theta_{\mathrm{qb}}^{\#}\right):=\left(\Lambda_{\mathrm{qb}, 1}^{\#}(p) v_{\mathrm{qb}}^{\#}, c(\epsilon)(D+2 \eta) \theta_{\mathrm{qb}}^{\#}\right)=\left(-\theta_{\mathrm{qb}}^{\#}, 0\right)+B_{\mathrm{qb}}^{\#}(p, \lambda)\left(v_{\mathrm{qb}}^{\#}, \theta_{\mathrm{qb}}^{\#}\right)$,

for $\#=-, \diamond-, \diamond+,+$. The differential operators are now given by

$$
\begin{aligned}
\Lambda_{\mathrm{qb}, 1}^{-}(p) v & =c(\epsilon)(D+2 \eta) v-L_{2 \eta}\left(s_{R}\left(\bar{w}_{\mathrm{qb}}(p), \epsilon\right)\right) v, \\
\Lambda_{\mathrm{qb}, 1}^{\#}(p) v & =c_{*}(D+2 \eta) v-L_{2 \eta}\left(q_{b}\right) v, \\
\Lambda_{\mathrm{qb}, 1}^{+}(p) v & =c(\epsilon)(D+2 \eta) v-L_{2 \eta}\left(s_{L}\left(\bar{w}_{\mathrm{qb}}(p), \epsilon\right)\right) v,
\end{aligned} \quad \text { for } \#=\diamond-, \diamond+,
$$

while the inhomogeneities can be written as

$$
\begin{aligned}
& B_{\mathrm{qb}, 1}^{-}(p, \lambda)(v, \theta)=-\lambda v+\left[g^{\prime}\left(\bar{u}_{\mathrm{qb}}(p)\right)-g^{\prime}\left(s_{R}\left(\bar{w}_{\mathrm{qb}}(p), \epsilon\right)\right)\right] v, \\
& B_{\mathrm{qb}, 1}^{\#}(p, \lambda)(v, \theta)=-\lambda v+\left[g^{\prime}\left(\bar{u}_{\mathrm{qb}}(p)\right)-g^{\prime}\left(q_{b}\right)\right] v \\
& +\frac{c_{*}-c(\epsilon)}{c(\epsilon)}\left[L_{2 \eta}\left(\bar{u}_{\mathrm{qb}}(p)\right) v-\theta-\lambda v\right], \quad \text { for } \#=\diamond-, \diamond+ \\
& B_{\mathrm{qf}, 1}^{+}(p, \lambda)(v, \theta)=-\lambda v+\left[g^{\prime}\left(\bar{u}_{\mathrm{qb}}(p)\right)-g^{\prime}\left(s_{L}\left(\bar{w}_{\mathrm{qb}}(p), \epsilon\right)\right)\right] v,
\end{aligned}
$$

together with

$$
B_{\mathrm{qb}, 2}^{\#}(p, \lambda)(v, \theta)=\epsilon(v-\gamma \theta)-\lambda \theta \quad \text { for } \#=-, \diamond-, \diamond+,+.
$$

Proposition 4.2 implies that for some constant $C>0$ we have the estimate

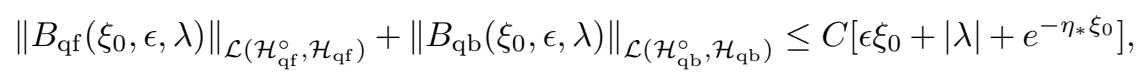

which holds for all $\xi_{0} \geq \xi_{*}$.

Lemma 6.1. Fix any sufficiently large constant $\xi_{*}$ and sufficiently small $\delta_{\tilde{\epsilon}}>0$. Choose any $\xi_{0} \geq \xi_{*}$. Then for any $p=\left(\xi_{0}, \epsilon\right) \in \mathcal{D}_{p}\left(\xi_{*}, \delta_{\tilde{\epsilon}}\right)$, there exist bounded 
linear operators

$$
\begin{array}{lll}
L_{\mathrm{qf}}(p) & : & \mathcal{H}_{\mathrm{qf}} \times \mathbb{C} \rightarrow \mathcal{H}_{\mathrm{qf}}^{\circ}, \\
L_{\mathrm{qb}}(p): & : & \mathcal{H}_{\mathrm{qb}} \times \mathbb{C} \rightarrow \mathcal{H}_{\mathrm{qb}}^{\circ}
\end{array}
$$

such that for any $g_{\mathrm{qf}}=\left(g_{\mathrm{qf}}^{-}, g_{\mathrm{qf}}^{\diamond}, g_{\mathrm{qf}}^{+}\right) \in \mathcal{H}_{\mathrm{qf}}, g_{\mathrm{qb}}=\left(g_{\mathrm{qf}}^{-}, g_{\mathrm{qf}}^{\diamond-}, g_{\mathrm{qf}}^{\diamond+}, g_{\mathrm{qf}}^{+}\right) \in \mathcal{H}_{\mathrm{qb}}$ and $\tilde{\kappa}_{f}, \tilde{\kappa}_{b} \in \mathbb{C}$, the sets of functions

$$
h_{\mathrm{qf}}=L_{\mathrm{qf}}(p)\left(g_{\mathrm{qf}}, \tilde{\kappa}_{f}\right) \in \mathcal{H}_{\mathrm{qf}}^{\circ}, \quad h_{\mathrm{qb}}=L_{\mathrm{qb}}(p)\left(g_{\mathrm{qb}}, \tilde{\kappa}_{b}\right) \in \mathcal{H}_{\mathrm{qb}}^{\circ}
$$

written as (6.7), are the unique sets that satisfy the following properties.

(i) The linear systems

$$
\begin{array}{lll}
\Lambda_{\mathrm{qf}}^{\#}(p)\left(v_{\mathrm{qf}}^{\#}, \theta_{\mathrm{qf}}^{\#}\right) & =\left(-\theta_{\mathrm{qf}}^{\#}, 0\right)+g_{\mathrm{qf}}^{\#}, & \#=-, \diamond,+, \\
\Lambda_{\mathrm{qb}}^{\#}(p)\left(v_{\mathrm{qb}}^{\#}, \theta_{\mathrm{qb}}^{\#}\right) & =\left(-\theta_{\mathrm{qb}}^{\#}, 0\right)+g_{\mathrm{qb}}^{\#}, & \#=-, \diamond-, \diamond+,+
\end{array}
$$

are all satisfied.

(ii) The continuity conditions $\theta_{\mathrm{qf}}^{-}(0)=\theta_{\mathrm{qf}}^{\diamond}(0), \theta_{\mathrm{qf}}^{\diamond}\left(\xi_{0}\right)=\theta_{\mathrm{qf}}^{+}\left(\xi_{0}\right), \theta_{\mathrm{qb}}^{-}\left(-\xi_{0}\right)=$ $\theta_{\mathrm{qb}}^{\diamond-}\left(-\xi_{0}\right), \theta_{\mathrm{qb}}^{\diamond-}(0)=\theta_{\mathrm{qb}}^{\diamond+}(0)$ and $\theta_{\mathrm{qb}}^{\diamond+}\left(\xi_{0}\right)=\theta_{\mathrm{qb}}^{+}\left(\xi_{0}\right)$ all hold.

(iii) The continuity conditions

$$
\mathrm{ev}_{\xi_{0}} v_{\mathrm{qf}}^{\diamond}=\mathrm{ev}_{\xi_{0}} v_{\mathrm{qf}}^{+}, \quad \quad \mathrm{ev}_{-\xi_{0}} v_{\mathrm{qb}}^{-}=\mathrm{ev}_{-\xi_{0}} v_{\mathrm{qb}}^{\diamond-}, \quad \mathrm{ev}_{\xi_{0}} v_{\mathrm{qb}}^{\diamond+}=\mathrm{ev}_{\xi_{0}} v_{\mathrm{qb}}^{+}
$$

all hold.

(iv) We have the jump conditions

$$
\mathrm{ev}_{0}\left[e_{2 \eta} v_{\mathrm{qf}}^{-}-e_{2 \eta} v_{\mathrm{qf}}^{\diamond}\right] \in \Gamma_{f}, \quad \mathrm{ev}_{0}\left[e_{2 \eta} v_{\mathrm{qb}}^{\diamond-}-e_{2 \eta} v_{\mathrm{qb}}^{\diamond+}\right] \in \Gamma_{b}
$$

(v) We have the boundary conditions

$$
\begin{aligned}
& \Pi_{B_{f}} \mathrm{ev}_{0} e_{2 \eta} v_{\mathrm{qf}}^{-}=\Pi_{B_{f}} \mathrm{ev}_{0} e_{2 \eta} v_{\mathrm{qf}}^{\diamond}=\tilde{\kappa}_{f} \mathrm{ev}_{0} q_{f}^{\prime}, \\
& \Pi_{B_{b}} \mathrm{ev}_{0} e_{2 \eta} v_{\mathrm{qb}}^{\diamond-}=\Pi_{B_{b}} \mathrm{ev}_{0} e_{2 \eta} v_{\mathrm{qb}}^{\diamond+}=\tilde{\kappa}_{b} \mathrm{ev}_{0} q_{b}^{\prime} .
\end{aligned}
$$

In addition, there exists a constant $N_{1}>0$ such that for any $\zeta>\delta_{\tilde{\epsilon}} N_{1}$, the maps

$$
\epsilon \mapsto\left\{\begin{array}{lll}
L_{\mathrm{qf}}\left(\xi_{0}, \epsilon\right) & \in \mathcal{L}\left(\mathcal{H}_{\mathrm{qf}} \times \mathbb{C}, \mathcal{H}_{\mathrm{qf}, 2 \zeta}^{\circ}\right), \\
L_{\mathrm{qb}}\left(\xi_{0}, \epsilon\right) & \in \mathcal{L}\left(\mathcal{H}_{\mathrm{qb}} \times \mathbb{C}, \mathcal{H}_{\mathrm{qb}, 2 \zeta}^{\circ}\right)
\end{array}\right.
$$

are $C^{2}$-smooth. For some constant $C>0$ we have the estimates

$$
\begin{aligned}
& \left\|L_{\mathrm{qf}}\left(\xi_{0}, \epsilon\right)\right\|_{\mathcal{L}\left(\mathcal{H}_{\mathrm{qf}} \times \mathbb{C}, \mathcal{H}_{\mathrm{qf}}^{\circ}\right)}+\left\|L_{\mathrm{qb}}\left(\xi_{0}, \epsilon\right)\right\|_{\mathcal{L}\left(\mathcal{H}_{\mathrm{qb}} \times \mathbb{C}, \mathcal{H}_{\mathrm{qb}}^{\circ}\right)} \leq C, \\
& \left\|D_{\epsilon} L_{\mathrm{qf}}\left(\xi_{0}, \epsilon\right)\right\|_{\mathcal{L}\left(\mathcal{H}_{\mathrm{qf}} \times \mathbb{C}, \mathcal{H}_{\mathrm{qf}, \zeta}^{\circ}\right)}+\left\|D_{\epsilon} L_{\mathrm{qb}}\left(\xi_{0}, \epsilon\right)\right\|_{\mathcal{L}\left(\mathcal{H}_{\mathrm{qb}} \times \mathbb{C}, \mathcal{H}_{\mathrm{qb}, \zeta}^{\circ}\right)} \leq C \xi_{0}, \\
& \left\|D_{\epsilon}^{2} L_{\mathrm{qf}}\left(\xi_{0}, \epsilon\right)\right\|_{\mathcal{L}\left(\mathcal{H}_{\mathrm{qf}} \times \mathbb{C}, \mathcal{H}_{\mathrm{qf}, 2 \zeta}^{\circ}\right)}+\left\|D_{\epsilon}^{2} L_{\mathrm{qb}}\left(\xi_{0}, \epsilon\right)\right\|_{\mathcal{L}\left(\mathcal{H}_{\mathrm{qb}} \times \mathbb{C}, \mathcal{H}_{\mathrm{qb}, 2 \zeta}^{\circ}\right)} \leq C \xi_{0}^{2},
\end{aligned}
$$


which hold for all $\left(\xi_{0}, \epsilon\right) \in \mathcal{D}_{p}$. Finally, consider any $d_{f} \in \mathbb{K}_{f}^{*}$ and $d_{b} \in \mathbb{K}_{b}^{*}$. Then the following identities hold for the gaps at zero,

$(6.26)$

$$
\begin{aligned}
\left\langle\mathrm{ev}_{0} d_{f}, \mathrm{ev}_{0} e_{2 \eta}\left[v_{\mathrm{qf}}^{-}-v_{\mathrm{qf}}^{\diamond}\right]\right\rangle= & \int_{-\infty}^{0} d_{f}\left(\xi^{\prime}\right)^{*} e^{2 \eta \xi^{\prime}} g_{\mathrm{qf}, 1}^{-}\left(\xi^{\prime}\right) \mathrm{d} \xi^{\prime} \\
& +\int_{0}^{\xi_{0}} d_{f}\left(\xi^{\prime}\right)^{*} e^{2 \eta \xi^{\prime}} g_{\mathrm{q},, 1}^{\diamond}\left(\xi^{\prime}\right) \mathrm{d} \xi^{\prime} \\
& -\frac{1}{c(\epsilon)} \int_{-\infty}^{0} d_{f}\left(\xi^{\prime}\right)^{*} \int_{-\infty}^{\xi^{\prime}} e^{2 \eta \xi^{\prime \prime}} g_{\mathrm{qf}, 2}^{-}\left(\xi^{\prime \prime}\right) \mathrm{d} \xi^{\prime \prime} \mathrm{d} \xi^{\prime} \\
& -\frac{1}{c(\epsilon)} \int_{0}^{\xi_{0}} d_{f}\left(\xi^{\prime}\right)^{*}\left[\int_{-\infty}^{0} e^{2 \eta \xi^{\prime \prime}} g_{\mathrm{qf}, 2}^{-}\left(\xi^{\prime \prime}\right) \mathrm{d} \xi^{\prime \prime}\right. \\
& \left.+\int_{0}^{\xi^{\prime}} e^{2 \eta \xi^{\prime \prime}} g_{\mathrm{qf}, 2}^{\diamond}\left(\xi^{\prime \prime}\right) \mathrm{d} \xi^{\prime \prime}\right] \mathrm{d} \xi^{\prime} \\
& -\left\langle\mathrm{ev}_{\xi_{0}} d_{f}, \mathrm{ev}_{\xi_{0}} e_{2 \eta} v_{\mathrm{qf}}^{\diamond}\right\rangle, \\
\left\langle\mathrm{ev}_{0} d_{b}, \mathrm{ev}_{0} e_{2 \eta}\left[v_{\mathrm{qb}}^{\diamond-}-v_{\mathrm{qb}}^{\diamond+}\right]\right\rangle= & \int_{-\xi_{0}}^{0} d_{b}\left(\xi^{\prime}\right)^{*} e^{2 \eta \xi^{\prime}} g_{\mathrm{qb}, 1}^{\diamond-}\left(\xi^{\prime}\right) \mathrm{d} \xi^{\prime} \\
& +\int_{0}^{\xi_{0}} d_{b}\left(\xi^{\prime}\right)^{*} e^{2 \eta \xi^{\prime}} g_{\mathrm{qb}, 1}^{\diamond+}\left(\xi^{\prime}\right) \mathrm{d} \xi^{\prime} \\
& -\frac{1}{c(\epsilon)} \int_{-\xi_{0}}^{0} d_{b}\left(\xi^{\prime}\right)^{*} \int_{-\xi_{0}}^{\xi^{\prime}} e^{2 \eta \xi^{\prime \prime}} g_{\mathrm{qb}, 2}^{\diamond-}\left(\xi^{\prime \prime}\right) \mathrm{d} \xi^{\prime \prime} \mathrm{d} \xi^{\prime} \\
& -\frac{1}{c(\epsilon)} \int_{0}^{\xi_{0}} d_{b}\left(\xi^{\prime}\right)^{*}\left[\int_{-\xi_{0}}^{0} e^{2 \eta \xi^{\prime \prime}} g_{\mathrm{qb}, 2}^{\diamond-,}\left(\xi^{\prime \prime}\right) \mathrm{d} \xi^{\prime \prime}\right. \\
& \left.+\int_{0}^{\xi^{\prime}} e^{2 \eta \xi^{\prime \prime}} g_{\mathrm{qb}, 2}^{\diamond+}\left(\xi^{\prime \prime}\right) \mathrm{d} \xi^{\prime \prime}\right] \mathrm{d} \xi^{\prime} \\
& -\left\langle\mathrm{ev}_{\xi_{0}} d_{b}, e \mathrm{ev}_{\xi_{0}} e_{2 \eta} v_{\mathrm{qb}}^{\diamond+}\right\rangle+\left\langle\mathrm{ev}_{-\xi_{0}} d_{b}, \mathrm{ev}-\xi_{0} e_{2 \eta} v_{\mathrm{qb}}^{\diamond-}\right\rangle .
\end{aligned}
$$

Proof. We will only discuss the operators $L_{\mathrm{qf}}$ here, noting that the statements concerning $L_{\mathrm{qb}}$ can be obtained in a similar fashion. The arguments are very similar to those used in the proof of [HS10, Lem. 5.10]. Indeed, we may use [HS10, Lem. 5.3] to define an operator

$$
\left[\Lambda_{1}^{+}(p)\right]^{-1}: B C_{-\eta}\left(\left[\xi_{0}, \infty\right), \mathbb{C}\right) \rightarrow B C_{-\eta}\left(\left[\xi_{0}-1, \infty\right), \mathbb{C}\right)
$$

and [HS10, Lem. 5.7] to define

$$
\begin{array}{lll}
{\left[\Lambda_{\mathrm{qf}, 1}^{-}\right]^{-1}} & : & B C_{-\eta}((-\infty, 0], \mathbb{C}) \rightarrow B C_{-\eta}((-\infty, 1], \mathbb{C}), \\
{\left[\Lambda_{\mathrm{qf}, 1}^{\diamond}\right]^{-1}} & : & B C_{0}\left(\left[0, \xi_{0}\right], \mathbb{C}\right) \rightarrow B C_{0}\left(\left[-1, \xi_{0}+1\right], \mathbb{C}\right),
\end{array}
$$

such that the choice $h_{0}=\left(v_{\mathrm{qf}, 0}^{-}, \theta_{\mathrm{qf}}^{-}, v_{\mathrm{qf}, 0}^{\diamond}, \theta_{\mathrm{qf}}^{\diamond}, v_{\mathrm{qf}, 0}^{+}, \theta_{\mathrm{qf}}^{+}\right)$with

$$
\begin{aligned}
\theta_{\mathrm{qf}}^{-}(\xi) & =\frac{1}{c(\epsilon)} e^{-2 \eta \xi} \int_{-\infty}^{\xi} e^{2 \eta \xi^{\prime}} g_{\mathrm{qf}, 2}^{-}\left(\xi^{\prime}\right) \mathrm{d} \xi^{\prime}, \\
\theta_{\mathrm{qf}}^{\diamond}(\xi) & =\theta_{\mathrm{qf}}^{-}(0) e^{-2 \eta \xi}+\frac{1}{c(\epsilon)} e^{-2 \eta \xi} \int_{0}^{\xi} e^{2 \eta \xi^{\prime}} g_{\mathrm{qf}, 2}^{\diamond}\left(\xi^{\prime}\right) \mathrm{d} \xi^{\prime}, \\
\theta_{\mathrm{qf}}^{+}(\xi) & =\theta_{\mathrm{qf}}^{\diamond}\left(\xi_{0}\right) e^{-2 \eta\left(\xi-\xi_{0}\right)}+\frac{1}{c(\epsilon)} e^{-2 \eta \xi} \int_{\xi_{0}}^{\xi} e^{2 \eta \xi^{\prime}} g_{\mathrm{qf}, 2}^{+}\left(\xi^{\prime}\right) \mathrm{d} \xi^{\prime}, \\
v_{\mathrm{qf}, 0}^{-} & =\left[\Lambda_{\mathrm{qf}, 1}^{-}\right]^{-1}\left[g_{\mathrm{qf}, 1}^{-}-\theta_{\mathrm{qf}}^{-}\right], \\
v_{\mathrm{qf}, 0}^{\diamond} & =\left[\Lambda_{\mathrm{qf}, 1}^{\diamond}\right]^{-1}\left[g_{\mathrm{qf}, 1}^{\diamond}-\theta_{\mathrm{qf}}^{\diamond}\right], \\
v_{\mathrm{qf}, 0}^{+} & =\left[\Lambda_{\mathrm{qf}, 1}^{+}(p)\right]^{-1}\left[g_{\mathrm{qf}, 1}^{+}-\theta_{\mathrm{qf}}^{+}\right]
\end{aligned}
$$

satisfies items (i) and (ii). We recall that the exponents -1 in (6.27)-6.28) are used suggestively, since the relevant homogeneous equations have nonzero solutions. This freedom can be used to ensure that the remaining properties (iii)-(v) are also satisfied. In particular, we will modify $v_{\mathrm{qf}, 0}^{-}, v_{\mathrm{qf}, 0}^{\diamond}$ and $v_{\mathrm{qf}, 0}^{+}$by choosing $\psi^{B \diamond} \in B_{f}$, $\psi^{B-} \in B_{f}, \psi^{\widehat{P}-} \in \widehat{P}_{f}, \psi^{\widehat{Q} \diamond} \in \widehat{Q}_{f}, \psi^{S \diamond} \in P_{R, 0}^{\mathrm{fb}}$ and $\psi^{Q+} \in Q_{R, 0}^{\mathrm{fb}}$ and writing

$$
\begin{aligned}
& v_{\mathrm{qf}}^{-}=v_{\mathrm{qf}, 0}^{-}+e_{-2 \eta} E^{P-}\left(\psi^{B-}+\psi^{\widehat{P}}-\right), \\
& v_{\mathrm{qf}}^{\diamond}=v_{\mathrm{qf}, 0}^{\diamond}+e_{-2 \eta} E^{Q \diamond}\left(\psi^{B \diamond}+\psi^{\widehat{Q} \diamond}\right)+e_{-2 \eta} E^{S \diamond}(p) \psi^{S \diamond}, \\
& v_{\mathrm{qf}}^{+}=v_{\mathrm{qf}, 0}^{+}+e_{-2 \eta} E^{Q+}(p) \psi^{Q+},
\end{aligned}
$$


in which the extension operators $E^{P-}, E^{Q \diamond}$ and $E^{S \diamond}$ are relabelled versions of those defined in [HS10, Lem. 5.9] for the unweighted linearizations (3.15). In addition, $E^{Q+}$ is constructed from the operator $E_{R, 0}^{\mathrm{fb}}$ appearing in [HS10, Lem. 5.5], again for the unweighted linearization (3.15).

In terms of these new variables, the continuity condition (iii) can be written as

$$
\begin{aligned}
& \phi_{P}=\psi^{S \diamond}+\Pi_{P_{R, 0}^{\mathrm{fb}}} \operatorname{ev}_{\xi_{0}} E^{Q \diamond}\left(\psi^{B \diamond}+\psi^{\widehat{Q} \diamond}\right)-\Pi_{P_{R, 0}^{\mathrm{fb}}} \mathrm{ev}_{\xi_{0}} E^{Q+}(p) \psi^{Q+}, \\
& \phi_{Q}=\psi^{Q+}-\Pi_{Q_{R, 0}^{\mathrm{fb}}} \operatorname{ev}_{\xi_{0}} E^{Q \diamond}\left(\psi^{B \diamond}+\psi^{\widehat{Q} \diamond}\right)+\Pi_{Q_{R, 0}^{\mathrm{fb}}} \operatorname{ev}_{\xi_{0}} E^{S \diamond} \psi^{S \diamond},
\end{aligned}
$$

in which

$$
\begin{aligned}
& \phi_{P}=-\Pi_{P_{R, 0}^{\mathrm{fb}}} \mathrm{ev}_{\xi_{0}} e_{2 \eta} v_{\mathrm{qf}, 0}^{\diamond}+\Pi_{P_{R, 0}^{\mathrm{fb}}} \mathrm{ev}_{\xi_{0}} e_{2 \eta} v_{\mathrm{qf}, 0}^{+}, \\
& \phi_{Q}=+\Pi_{Q_{R, 0}^{\mathrm{fb}}} \operatorname{ev} v_{\xi_{0}} e_{2 \eta} v_{\mathrm{qf}, 0}^{\diamond}-\Pi_{Q_{R, 0}^{\mathrm{fb}}} \operatorname{ev}_{\xi_{0}} e_{2 \eta} v_{\mathrm{qf}, 0}^{+} .
\end{aligned}
$$

Conditions (iv) and (v) are equivalent to the system

$$
\begin{aligned}
& -\Pi_{B_{f}} \mathrm{ev}_{0} e_{2 \eta} v_{\mathrm{qf}, 0}^{-}+\tilde{\kappa}_{f} \mathrm{ev}_{0} q_{f}^{\prime}=\psi^{B-}, \\
& -\Pi_{B_{f}} \mathrm{ev}_{0} e_{2 \eta} v_{\mathrm{qf}, 0}^{\diamond}+\tilde{\kappa}_{f} \mathrm{ev}_{0} q_{f}^{\prime}=\psi^{B \diamond}+\Pi_{B_{f}} \mathrm{ev}_{0} E^{S \diamond} \psi^{S \diamond} \text {, } \\
& -\Pi_{\widehat{P}_{f}} \mathrm{ev}_{0} e_{2 \eta}\left(v_{\mathrm{qf}, 0}^{-}-v_{\mathrm{qf}, 0}^{\diamond}\right)=\psi^{\widehat{P}-}-\Pi_{\widehat{P}_{f}} \mathrm{ev}_{0} E^{S \diamond} \psi^{S \diamond}, \\
& \Pi_{\widehat{Q}_{f}} \mathrm{ev}_{0} e_{2 \eta}\left(v_{\mathrm{qf}, 0}^{-}-v_{\mathrm{qf}, 0}^{\diamond}\right)=\psi^{\widehat{Q} \diamond}+\Pi_{\widehat{Q}_{f}} \mathrm{ev}_{0} E^{S \diamond} \psi^{S \diamond} \text {. }
\end{aligned}
$$

Inspection of the system 6.31 - 6.33) readily shows that for sufficiently large $\xi_{*}$ and sufficiently small $\delta_{\tilde{\epsilon}}$, the right hand side is close to the identity matrix, allowing the system to be solved. To complete the proof, observe that the integral expressions (6.26) follow from (3.36), while the smoothness of the maps (6.24) follows from HS10, Lem. 5.3 and 5.5].

In order to construct the functions $\left(u_{\mathrm{qf}}^{ \pm}, w_{\mathrm{qf}}\right)$ featured in Proposition 5.5, it now suffices to solve the fixed point problem

$$
h_{\mathrm{qf}}=L_{\mathrm{qf}}(p)\left(B_{\mathrm{qf}}^{-}(p, \lambda) h_{\mathrm{qf}}, B_{\mathrm{qf}}^{\diamond}(p, \lambda) h_{\mathrm{qf}}, B_{\mathrm{qf}}^{+}(p, \lambda) h_{\mathrm{qf}}, 0\right)+L_{1}\left(\xi_{0}, \epsilon\right)(0,1),
$$

for $\left(\xi_{0}, \epsilon\right) \in \mathcal{D}_{p}\left(\xi_{*}, \delta_{\tilde{\epsilon}}\right)$ and $|\lambda|<\delta_{\lambda}$. In view of the bound (6.17), the constants $\xi_{*}, \delta_{\tilde{\epsilon}}$ and $\delta_{\lambda}$ can be chosen in such a way that this problem has a unique solution which we will denote by $h_{\mathrm{qf}}^{*}\left(\xi_{0}, \epsilon, \lambda\right)$. The triplet $\left(u_{\mathrm{qf}}^{-}, u_{\mathrm{qf}}^{+}, w_{\mathrm{qf}}\right)$ can subsequently be constructed from $h_{\mathrm{qf}}^{*}$ using (6.8)- - (6.9). Of course, a similar construction can be used to obtain the corresponding functions $h_{\mathrm{qb}}^{*}$ and $\left(u_{\mathrm{qb}}^{-}, u_{\mathrm{qb}}^{+}, w_{\mathrm{qb}}\right)$.

It remains to establish the Melnikov identities (5.5). To this end, notice that $h_{\mathrm{qf}}^{*}\left(\xi_{0}, 0,0\right)=e_{-2 \eta} q_{f}^{\prime}$. An easy computation now shows that the identities

$$
\begin{aligned}
& {\left[D_{\lambda} B_{\mathrm{qf}, 1}^{\#}\left(\xi_{0}, 0,0\right)\right] h_{\mathrm{qf}}^{*}\left(\xi_{0}, 0,0\right)=-e_{-2 \eta} q_{f}^{\prime},} \\
& {\left[D_{\lambda} B_{\mathrm{qf}, 2}^{\#}\left(\xi_{0}, 0,0\right)\right] h_{\mathrm{qf}}^{*}\left(\xi_{0}, 0,0\right)=0}
\end{aligned}
$$

hold for \# $=-, \diamond$. The Melnikov identity in (5.35) for $M_{f, \lambda}$ now follows easily from (6.26). Turning to $M_{f, \epsilon}$, we observe that

$$
\begin{aligned}
{\left[D_{\epsilon} B_{\mathrm{qf}, 1}^{\#}\left(\xi_{0}, 0,0\right)\right] h_{\mathrm{qf}}^{*}\left(\xi_{0}, 0,0\right) } & =e_{-2 \eta}\left[g^{\prime \prime}\left(q_{f}\right) D_{\epsilon} \bar{u}_{\mathrm{qf}}\left(\xi_{0}, 0\right) q_{f}^{\prime}-c_{*}^{-1} D_{\epsilon} c(0) L\left(q_{f}\right) q_{f}^{\prime}\right], \\
& =e_{-2 \eta}\left[g^{\prime \prime}\left(q_{f}\right) D_{\epsilon} \bar{u}_{\mathrm{qf}}\left(\xi_{0}, 0\right) q_{f}^{\prime}-D_{\epsilon} c(0) q_{f}^{\prime \prime}\right], \\
{\left[D_{\epsilon} B_{\mathrm{qf}, 2}^{\#}\left(\xi_{0}, 0,0\right)\right] h_{\mathrm{qf}}^{*}\left(\xi_{0}, 0,0\right) } & =e_{-2 \eta} q_{f}^{\prime},
\end{aligned}
$$


for both $\#=-, \diamond$. By definition, we also have

$$
\begin{aligned}
& c(\epsilon) \bar{u}_{\mathrm{qf}}^{\prime \prime}\left(\xi_{0}, \epsilon\right)=L\left(\bar{u}_{\mathrm{qf}}\left(\xi_{0}, \epsilon\right)\right) \bar{u}_{\mathrm{qf}}^{\prime}\left(\xi_{0}, \epsilon\right)-\bar{w}_{\mathrm{qf}}^{\prime}\left(\xi_{0}, \epsilon\right), \\
& c(\epsilon) \bar{w}_{\mathrm{qf}}^{\prime}\left(\xi_{0}, \epsilon\right)=\epsilon\left(\bar{u}_{\mathrm{qf}}\left(\xi_{0}, \epsilon\right)-\gamma \bar{w}_{\mathrm{qf}}\left(\xi_{0}, \epsilon\right)\right) .
\end{aligned}
$$

Differentiating these identities with respect to $\epsilon$ and evaluating them at $\epsilon=0$, we find

$$
c_{*} D_{\epsilon} \bar{w}_{\mathrm{qf}}^{\prime}\left(\xi_{0}, 0\right)=q_{f}
$$

and also

$$
\Lambda_{f} D_{\epsilon} \bar{u}_{\mathrm{qf}}^{\prime}\left(\xi_{0}, 0\right)=g^{\prime \prime}\left(q_{f}\right) D_{\epsilon} \bar{u}_{\mathrm{qf}}\left(\xi_{0}, 0\right) q_{f}^{\prime}-D_{\epsilon} c(0) q_{f}^{\prime \prime}-c_{*}^{-1} q_{f} .
$$

Using (6.26) we now compute $(6.40)$

$$
\begin{aligned}
M_{f, \epsilon} & =\int_{-\infty}^{\infty} d_{f}(\xi)^{*}\left[g^{\prime \prime}\left(q_{f}(\xi)\right) D_{\epsilon} \bar{u}_{\mathrm{qf}}\left(\xi_{0}, 0\right)(\xi) q_{f}^{\prime}(\xi)-D_{\epsilon} c(0) q_{f}^{\prime \prime}(\xi)-c_{*}^{-1} q_{f}(\xi)\right] d \xi \\
& =0
\end{aligned}
$$

in which the second identity follows from the fact that $d_{f} \in \mathbb{K}_{f}^{*}$.

The Melnikov identity in (5.35) for $M_{b, \lambda}$ follows from the computation

$$
\begin{aligned}
& {\left[D_{\lambda} B_{\mathrm{qb}, 1}^{\#}\left(\xi_{0}, 0,0\right)\right] h_{\mathrm{qb}}^{*}\left(\xi_{0}, 0,0\right)=-e_{-2 \eta} q_{b}^{\prime},} \\
& {\left[D_{\lambda} B_{\mathrm{qb}, 2}^{\#}\left(\xi_{0}, 0,0\right)\right] h_{\mathrm{qb}}^{*}\left(\xi_{0}, 0,0\right)=0}
\end{aligned}
$$

which holds for $\#=\diamond-, \diamond+$. To compute $M_{b, \epsilon}$, note that

$$
\begin{aligned}
{\left[D_{\epsilon} B_{\mathrm{qb}, 1}^{\#}\left(\xi_{0}, 0,0\right)\right] h_{\mathrm{qb}}^{*}\left(\xi_{0}, 0,0\right) } & =e_{-2 \eta}\left[g^{\prime \prime}\left(q_{b}\right) D_{\epsilon} \bar{u}_{\mathrm{qb}}\left(\xi_{0}, 0\right) q_{b}^{\prime}-c_{*}^{-1} D_{\epsilon} c(0) L\left(q_{b}\right) q_{b}^{\prime}\right] \\
& =e_{-2 \eta}\left[g^{\prime \prime}\left(q_{b}\right) D_{\epsilon} \bar{u}_{\mathrm{qb}}\left(\xi_{0}, 0\right) q_{b}^{\prime}-D_{\epsilon} c(0) L\left(q_{b}\right) q_{b}^{\prime \prime}\right], \\
{\left[D_{\epsilon} B_{\mathrm{qb}, 2}^{\#}\left(\xi_{0}, 0,0\right)\right] h_{\mathrm{qb}}^{*}\left(\xi_{0}, 0,0\right) } & =e_{-2 \eta} q_{b}^{\prime},
\end{aligned}
$$

again for $\#=\diamond-, \diamond+$. As above, we recall the definitions

$$
\begin{aligned}
& c(\epsilon) \bar{u}_{\mathrm{qb}}^{\prime \prime}\left(\xi_{0}, \epsilon\right)=L\left(\bar{u}_{\mathrm{qb}}\left(\xi_{0}, \epsilon\right)\right) \bar{u}_{\mathrm{qb}}^{\prime}\left(\xi_{0}, \epsilon\right)-\bar{w}_{\mathrm{qb}}^{\prime}\left(\xi_{0}, \epsilon\right) \\
& c(\epsilon) \bar{w}_{\mathrm{qb}}^{\prime}\left(\xi_{0}, \epsilon\right)=\epsilon\left(\bar{u}_{\mathrm{qb}}\left(\xi_{0}, \epsilon\right)-\gamma \bar{w}_{\mathrm{qb}}\left(\xi_{0}, \epsilon\right)\right)
\end{aligned}
$$

and differentiate with respect to $\epsilon$. Evaluating the result at $\epsilon=0$, we find

$$
c_{*} D_{\epsilon} \bar{w}_{\mathrm{qb}}^{\prime}\left(\xi_{0}, 0\right)=q_{b}-\gamma w_{*}
$$

and also

$$
\Lambda_{b} D_{\epsilon} \bar{u}_{\mathrm{qb}}^{\prime}\left(\xi_{0}, 0\right)=g^{\prime \prime}\left(q_{b}\right) D_{\epsilon} \bar{u}_{\mathrm{qb}}\left(\xi_{0}, 0\right) q_{b}^{\prime}-D_{\epsilon} c(0) q_{b}^{\prime \prime}-c_{*}^{-1}\left[q_{b}-\gamma w_{*}\right]
$$

which allows us to compute

$$
\begin{aligned}
M_{b, \epsilon}=\int_{-\infty}^{\infty} d_{b}(\xi)^{*}[ & g^{\prime \prime}\left(q_{b}(\xi)\right) D_{\epsilon} \bar{u}_{\mathrm{qb}}\left(\xi_{0}, 0\right)(\xi) q_{b}^{\prime}(\xi) \\
& \left.\quad-D_{\epsilon} c(0) q_{b}^{\prime \prime}(\xi)-c_{*}^{-1}\left[q_{b}(\xi)-\widetilde{s}_{R}\left(w_{*}\right)\right]\right] d \xi \\
=\frac{1}{c_{*}} \int_{-\infty}^{\infty} d_{b}(\xi)^{*} & {\left[\widetilde{s}_{R}\left(w_{*}\right)-\gamma w_{*}\right] d \xi . }
\end{aligned}
$$

With these calculations the proof of Proposition 5.5 is complete. 
6.2. Region $R_{1}$ : Exchange Lemma. In this part we set out to prove Proposition 5.6. We will reuse many of the function spaces from the previous section, but we will also need the families

$(6.47)$

$$
\begin{aligned}
& B C_{\mathrm{qsf}, \beta}^{\oplus}=\left\{(v, \theta) \in C\left(\left[\xi_{0}-1, \frac{1}{2} T+1\right], \mathbb{C}\right) \times C\left(\left[\xi_{0}, \frac{1}{2} T\right], \mathbb{C}\right)\right. \text { for which } \\
& \|(v, \theta)\|_{B C_{\mathrm{qsf}, \beta}^{\oplus}}:=\sup _{\xi_{0}-1 \leq \xi \leq \frac{1}{2} T+1} \mathrm{e}^{-\beta\left|\xi-\xi_{0}\right|}|v(\xi)| \\
& \left.+\sup _{\xi_{0} \leq \xi \leq \frac{1}{2} T} \mathrm{e}^{-\beta\left|\xi-\xi_{0}\right|}|\theta(\xi)|<\infty\right\}, \\
& B C_{\mathrm{qsb}, \beta}^{\ominus}=\left\{(v, \theta) \in C\left(\left[-\frac{1}{2} T-1,-\xi_{0}+1\right], \mathbb{C}\right) \times C\left(\left[-\frac{1}{2} T,-\xi_{0}\right], \mathbb{C}\right)\right. \text { for which } \\
& \|(v, \theta)\|_{B C_{\mathrm{qsb}, \beta}^{\ominus}}:=\sup _{-\frac{1}{2} T-1 \leq \xi \leq-\xi_{0}+1} \mathrm{e}^{-\beta\left|\xi+\xi_{0}\right|}|v(\xi)| \\
& \left.+\sup _{-\frac{1}{2} T \leq \xi \leq-\xi_{0}} \mathrm{e}^{-\beta\left|\xi+\xi_{0}\right|}|\theta(\xi)|<\infty\right\},
\end{aligned}
$$

together with

(6.48)

$$
\begin{array}{cc}
B C_{\mathrm{qsf}, \beta}^{+}= & \left\{(v, \theta) \in C\left(\left[\xi_{0}, \frac{1}{2} T\right], \mathbb{C}\right) \times C\left(\left[\xi_{0}, \frac{1}{2} T\right], \mathbb{C}\right)\right. \text { for which } \\
\left.\|(v, \theta)\|_{B C_{\mathrm{qsf}, \beta}^{+}}:=\sup _{\xi_{0} \leq \xi \leq \frac{1}{2} T} \mathrm{e}^{-\beta\left|\xi-\xi_{0}\right|}(|v(\xi)|+|\theta(\xi)|)<\infty\right\}, \\
B C_{\mathrm{qsb}, \beta}^{-}= & \left\{(v, \theta) \in C\left(\left[-\frac{1}{2} T,-\xi_{0}\right], \mathbb{C}\right) \times C\left(\left[-\frac{1}{2} T,-\xi_{0}\right], \mathbb{C}\right)\right. \text { for which } \\
\left.\|(v, \theta)\|_{B C_{\mathrm{qsb}, \beta}^{-}}:=\sup _{-\frac{1}{2} T \leq \xi \leq-\xi_{0}} \mathrm{e}^{-\beta\left|\xi+\xi_{0}\right|}(|v(\xi)|+|\theta(\xi)|)<\infty\right\},
\end{array}
$$

that are both parametrized by $\beta \in \mathbb{R}$.

We also introduce the family of composite function spaces

$$
\mathcal{H}_{\mathrm{qs}, \zeta}^{\circ}=B C_{\mathrm{qf},-\eta}^{\ominus} \times B C_{\mathrm{qf}}^{\odot} \times B C_{\mathrm{qsf}, \eta}^{\oplus} \times B C_{\mathrm{qsb}, \eta}^{\ominus} \times B C_{\mathrm{qb}}^{\odot-} \times B C_{\mathrm{qb}}^{\odot+} \times B C_{\mathrm{qb},-\eta+\zeta}^{\oplus}
$$

parametrized by $\zeta \geq 0$, together with

$$
\mathcal{H}_{\mathrm{qs}}=B C_{\mathrm{qf},-\eta}^{-} \times B C_{\mathrm{qf}}^{\diamond} \times B C_{\mathrm{qsf}, \eta}^{+} \times B C_{\mathrm{qsb}, \eta}^{-} \times B C_{\mathrm{qb}}^{\diamond-} \times B C_{\mathrm{qb}}^{\diamond+} \times B C_{\mathrm{qb},-\eta}^{+}
$$

It is important to note that we are using positive weights in the function spaces that describe the passage near $\mathcal{M}_{R}$. This will allow us to establish the exponential estimates in (5.43).

Our goal is to find

$$
h_{\mathrm{qs}}=\left(v_{f}^{-}, \theta_{f}^{-}, v_{f}^{\diamond}, \theta_{f}^{\diamond}, v_{f}^{+}, \theta_{f}^{+}, v_{b}^{-}, \theta_{b}^{-}, v_{b}^{\diamond-}, \theta_{b}^{\diamond-}, v_{b}^{\diamond+}, \theta_{b}^{\diamond+}, v_{b}^{+}, \theta_{b}^{+}\right) \in \mathcal{H}_{\mathrm{qs}}^{\circ},
$$

such that the choices

$$
w_{\mathrm{qs}}(\xi)= \begin{cases}w_{\mathrm{qf}}\left(\xi_{0}, T^{\mathrm{sl}} / T, \lambda\right)(\xi)+\theta_{f}^{-}(\xi) & \text { for } \xi \leq 0, \\ w_{\mathrm{qf}}\left(\xi_{0}, T^{\mathrm{sl}} / T, \lambda\right)(\xi)+\theta_{f}^{\diamond}(\xi) & \text { for } 0 \leq \xi \leq \xi_{0}, \\ w_{\mathrm{qf}}\left(\xi_{0}, T^{\mathrm{sl}} / T, \lambda\right)(\xi)+\theta_{f}^{+}(\xi) & \text { for } \xi_{0} \leq \xi \leq \frac{1}{2} T, \\ w_{\mathrm{qb}}\left(\xi_{0}, T^{\mathrm{sl}} / T, \lambda\right)(\xi-T)+\theta_{b}^{-}(\xi-T) & \text { for } \frac{1}{2} T \leq \xi \leq T-\xi_{0}, \\ w_{\mathrm{qb}}\left(\xi_{0}, T^{\mathrm{sl}} / T, \lambda\right)(\xi-T)+\theta_{b}^{\diamond}(\xi-T) & \text { for } T-\xi_{0} \leq \xi \leq T, \\ w_{\mathrm{qb}}\left(\xi_{0}, T^{\mathrm{sl}} / T, \lambda\right)(\xi-T)+\theta_{b}^{\diamond+}(\xi-T) & \text { for } T \leq \xi \leq T+\xi_{0}, \\ w_{\mathrm{qb}}\left(\xi_{0}, T^{\mathrm{sl}} / T, \lambda\right)(\xi-T)+\theta_{b}^{+}(\xi-T) & \text { for } \xi \geq T+\xi_{0},\end{cases}
$$


in combination with $u_{\mathrm{qsf}}=u_{\mathrm{qf}}^{-}\left(\xi_{0}, T^{\mathrm{sl}} / T, \lambda\right)+v_{f}^{-}$and

$$
\begin{aligned}
& u_{\mathrm{qsb}}(\xi)= \begin{cases}u_{\mathrm{qb}}^{+}\left(\xi_{0}, T^{\mathrm{sl}} / T, \lambda\right)(\xi-T)+v_{b}^{\diamond+}(\xi-T) & \text { for } T-1 \leq \xi \leq T+\xi_{0}, \\
u_{\mathrm{qb}}^{+}\left(\xi_{0}, T^{\mathrm{sl}} / T, \lambda\right)(\xi-T)+v_{b}^{+}(\xi-T) & \text { for } \xi \geq T+\xi_{0},\end{cases} \\
& u_{\mathrm{qsx}}(\xi)= \begin{cases}u_{\mathrm{qf}}^{+}\left(\xi_{0}, T^{\mathrm{sl}} / T, \lambda\right)(\xi)+v_{f}^{\diamond}(\xi) & \text { for }-1 \leq \xi \leq \xi_{0}, \\
u_{\mathrm{qf}}^{+}\left(\xi_{0}, T^{\mathrm{sl}} / T, \lambda\right)(\xi)+v_{f}^{+}(\xi) & \text { for } \xi_{0} \leq \xi \leq \frac{1}{2} T, \\
u_{\mathrm{qb}}^{-}\left(\xi_{0}, T^{\mathrm{sl}} / T, \lambda\right)(\xi-T)+v_{b}^{-}(\xi-T) & \text { for } \frac{1}{2} T \leq \xi \leq T-\xi_{0}, \\
u_{\mathrm{qb}}^{-}\left(\xi_{0}, T^{\mathrm{sl}} / T, \lambda\right)(\xi-T)+v_{b}^{\diamond-}(\xi-T) & \text { for } T-\xi_{0} \leq \xi \leq T,\end{cases}
\end{aligned}
$$

satisfies the conditions of Proposition 5.6 .

Plugging this Ansatz into (5.37), we find that we must solve the equations

$$
\begin{aligned}
\Lambda_{\mathrm{qsf}}^{\#}(\omega)\left(v_{f}^{\#}, \theta_{f}^{\#}\right):=\left(\Lambda_{\mathrm{qsf}, 1}^{\#}(\omega) v_{f}^{\#}, c(\omega)(D+2 \eta) \theta_{f}^{\#}\right) \\
=\left(-\theta_{f}^{\#}, 0\right)+B_{\mathrm{qsf}}^{\#}(\omega, \lambda)\left(v_{f}^{\#}, \theta_{f}^{\#}\right)-\lambda\left(v_{f}^{\#}, \theta_{f}^{\#}\right)+\mathcal{M}_{\mathrm{qsf}}^{\#}(\omega, \lambda) \tilde{\kappa}_{f}
\end{aligned}
$$

for $\#=-, \diamond,+$, together with

$(6.55)$

$$
\begin{aligned}
\Lambda_{\mathrm{qsb}}^{\#}(\omega)\left(v_{b}^{\#}, \theta_{b}^{\#}\right):=\left(\Lambda_{\mathrm{qsb}, 1}^{\#}(\omega) v_{b}^{\#}, c(\omega)(D+2 \eta) \theta_{b}^{\#}\right) \\
=\left(-\theta_{b}^{\#}, 0\right)+B_{\mathrm{qsb}}^{\#}(\omega, \lambda)\left(v_{b}^{\#}, \theta_{b}^{\#}\right)-\lambda\left(v_{b}^{\#}, \theta_{b}^{\#}\right)+\mathcal{M}_{\mathrm{qsb}}^{\#}(\omega, \lambda) \tilde{\kappa}_{b}
\end{aligned}
$$

for $\#=-, \diamond-, \diamond+,+$. Here we have

$$
\begin{array}{lll}
\Lambda_{\mathrm{qsf}, 1}^{\#}(\omega) v & =c_{*}(D+2 \eta) v-L\left(q_{f}\right) v, & \text { for } \#=-, \diamond, \\
\Lambda_{\mathrm{qsf}, 1}^{+}(\omega) v & =c(\omega)(D+2 \eta) v-L\left(s_{R}\left(\bar{w}_{\mathrm{qs}}(\omega), \epsilon\right)\right) v, & \\
\Lambda_{\mathrm{qsb}, 1}^{-}(\omega) v & =c(\omega)(D+2 \eta) v-L\left(s_{R}\left(\bar{w}_{\mathrm{qs}}(\omega), \epsilon\right)\right) v, & \\
\Lambda_{\mathrm{qsb}, 1}^{\#}(\omega) v & =c_{*}(D+2 \eta) v-L\left(q_{b}\right) v, & \\
\Lambda_{\mathrm{qsb}, 1}^{+}(\omega) v & =c(\omega)(D+2 \eta) v-L\left(s_{L}\left(\bar{w}_{\mathrm{qs}}(\omega), \epsilon\right)\right) v . &
\end{array}
$$

The inhomogeneities are given by

$$
\begin{aligned}
B_{\mathrm{qsf}, 1}^{-}(\omega, \lambda)(v, \theta)= & {\left[g^{\prime}\left(\bar{u}_{\mathrm{qsf}}(\omega)\right)-g^{\prime}\left(q_{f}\right)\right] v } \\
& +\frac{c_{*}-c(\epsilon)}{c(\epsilon)}\left[L\left(\bar{u}_{\mathrm{qsf}}(\omega)\right)-\theta-\lambda v\right], \\
B_{\mathrm{qsf}, 1}^{\diamond}(\omega, \lambda)(v, \theta)= & {\left[g^{\prime}\left(\bar{u}_{\mathrm{qsx}}(\omega)\right)-g^{\prime}\left(q_{f}\right)\right] v } \\
& +\frac{c_{*}-c(\epsilon)}{c(\epsilon)}\left[L\left(\bar{u}_{\mathrm{qsx}}(\omega)\right)-\theta-\lambda v\right], \\
B_{\mathrm{qsf}, 1}^{+}(\omega, \lambda)(v, \theta)= & {\left[g^{\prime}\left(\bar{u}_{\mathrm{qsx}}(\omega)\right)-g^{\prime}\left(s_{R}\left(\bar{w}_{\mathrm{qs}}(\omega), \epsilon\right)\right] v,\right.} \\
B_{\mathrm{qsb}, 1}^{-}(\omega, \lambda)(v, \theta)= & {\left[g^{\prime}\left(\bar{u}_{\mathrm{qsx}}(\omega)\right)-g^{\prime}\left(s_{R}\left(\bar{w}_{\mathrm{qs}}(\omega), \epsilon\right)\right] v,\right.} \\
B_{\mathrm{qsb}, 1}^{\diamond-}(\omega, \lambda)(v, \theta)= & {\left[g^{\prime}\left(\bar{u}_{\mathrm{qsx}}(\omega)\right)-g^{\prime}\left(q_{b}\right)\right] v } \\
& +\frac{c_{*}-c(\epsilon)}{c(\epsilon)}\left[L\left(\bar{u}_{\mathrm{qsx}}(\omega)\right)-\theta-\lambda\right], \\
B_{\mathrm{qsb}, 1}^{\diamond+}(\omega, \lambda)(v, \theta)= & {\left[g^{\prime}\left(\bar{u}_{\mathrm{qsb}}(\omega)\right)-g^{\prime}\left(q_{b}\right)\right] v } \\
& +\frac{c_{*}-c(\epsilon)}{c(\epsilon)}\left[L\left(\bar{u}_{\mathrm{qsb}}(\omega)\right)-\theta-\lambda\right], \\
B_{\mathrm{qsb}, 1}^{+}(\omega, \lambda)(v, \theta)= & {\left[g^{\prime}\left(\bar{u}_{\mathrm{qsb}}(\omega)\right)-g^{\prime}\left(s_{L}\left(\bar{w}_{\mathrm{qs}}(\omega), \epsilon\right)\right)\right] v, }
\end{aligned}
$$

together with

$$
\begin{aligned}
& B_{\mathrm{qsf}, 2}^{\#}(\omega, \lambda)(v, \theta)=\epsilon(v-\gamma \theta) \quad \text { for } \#=-, \diamond,+, \\
& B_{\mathrm{qsb}, 2}^{\#}(\omega, \lambda)(v, \theta)=\epsilon(v-\gamma \theta) \quad \text { for } \#=-, \diamond-, \diamond+,+ \text {. }
\end{aligned}
$$

The reason that we do not include the terms $\lambda\left(v_{f}^{\#}, \theta_{f}^{\#}\right)$ in the inhomogeneity $B_{\mathrm{qsf}}^{\#}$ is to ease our notation in the next section. We combine these inhomogeneities into 
the single entity

$$
B_{\mathrm{qs}}=\left(B_{\mathrm{qsf}}^{-}, B_{\mathrm{qsf}}^{\diamond}, B_{\mathrm{qsf}}^{+}, B_{\mathrm{qsb}}^{-}, B_{\mathrm{qsb}}^{\diamond-}, B_{\mathrm{qsb}}^{\diamond+}, B_{\mathrm{qsb}}^{+}\right) \in \mathcal{L}\left(\mathcal{H}_{\mathrm{qs}}^{\circ}, H_{\mathrm{qs}}\right) .
$$

Using Propositions 4.2 and 4.3 , we obtain the bound

$$
\left\|B_{\mathrm{qs}}(\omega, \lambda)\right\|_{\mathcal{L}\left(\mathcal{H}_{\mathrm{qs}}^{\circ}, \mathcal{H}_{\mathrm{qs}}\right)} \leq C\left[\epsilon \xi_{0}+e^{-\eta_{*} \xi_{0}}+e^{-\frac{1}{2} \eta T}\right] .
$$

The inhomogeneous terms in (6.54)-(6.55) are given by

$$
\begin{array}{lll}
\mathcal{M}_{\mathrm{qgf}, 1}^{-}(\omega, \lambda)=\left[g^{\prime}\left(\bar{u}_{\mathrm{qsf}}(\omega)\right)-g^{\prime}\left(\bar{u}_{\mathrm{qf}}\left(\xi_{0}, \epsilon\right)\right)\right] u_{\mathrm{qf}}\left(\xi_{0}, \epsilon, \lambda\right), & \\
\mathcal{M}_{\mathrm{qgf}, 1}^{\#}(\omega, \lambda)=\left[g^{\prime}\left(\bar{u}_{\mathrm{qsx}}(\omega)\right)-g^{\prime}\left(\bar{u}_{\mathrm{qf}}\left(\xi_{0}, \epsilon\right)\right)\right] u_{\mathrm{qf}}\left(\xi_{0}, \epsilon, \lambda\right), & & \text { for } \#=\diamond,+, \\
\mathcal{M}_{\mathrm{qgb}, 1}^{\#}(\omega, \lambda)=\left[g^{\prime}\left(\bar{u}_{\mathrm{qsx}}(\omega)\right)-g^{\prime}\left(\bar{u}_{\mathrm{qb}}\left(\xi_{0}, \epsilon\right)\right)\right] u_{\mathrm{qb}}\left(\xi_{0}, \epsilon, \lambda\right), & & \text { for } \#=-, \diamond-, \\
\mathcal{M}_{\mathrm{qsb}, 1}^{\#}(\omega, \lambda)=\left[g^{\prime}\left(\bar{u}_{\mathrm{qsb}}(\omega)\right)-g^{\prime}\left(\bar{u}_{\mathrm{qb}}\left(\xi_{0}, \epsilon\right)\right)\right] u_{\mathrm{qb}}\left(\xi_{0}, \epsilon, \lambda\right), & & \text { for } \#=\diamond+,+,
\end{array}
$$

with vanishing second components. Combining these into a single entity

$$
\mathcal{M}_{\mathrm{qs}}=\left(\mathcal{M}_{\mathrm{qsf}}^{-}, \mathcal{M}_{\mathrm{qsf}}^{\diamond}, \mathcal{M}_{\mathrm{qsf}}^{+}, \mathcal{M}_{\mathrm{qsb}}^{-}, \mathcal{M}_{\mathrm{qsb}}^{\diamond-}, \mathcal{M}_{\mathrm{qsb}}^{\diamond+}, \mathcal{M}_{\mathrm{qsb}}^{+}\right) \in \mathcal{H}_{\mathrm{qs}},
$$

we may invoke Proposition 4.3 to obtain the estimate

$$
\left\|\mathcal{M}_{\mathrm{qs}}(\omega, \lambda)\right\|_{\mathcal{H}_{\mathrm{qs}}}+\left\|D_{T^{\mathrm{sl}}} \mathcal{M}_{\mathrm{qs}}(\omega, \lambda)\right\|_{\mathcal{H}_{\mathrm{qs}}} \leq C e^{-\eta T}
$$

for some $C>0$.

Lemma 6.2. Fix sufficiently large constants $\xi_{*}$ and $M_{T}$ and a sufficiently small constant $\delta_{\mathrm{sl}}>0$. Then for any $\omega=\left(\xi_{0}, T^{\mathrm{sl}}, T\right) \in \Omega=\Omega\left(\xi_{*}, \delta_{\mathrm{sl}}, M_{T}\right)$, there exists a bounded linear map

$$
L_{\mathrm{qs}}(\omega): \mathcal{H}_{\mathrm{qs}} \times C([-1,1], \mathbb{C}) \times \mathbb{C} \rightarrow \mathcal{H}_{\mathrm{qs}}^{\circ}
$$

such that for any set

$$
g_{\mathrm{qs}}=\left(g_{f}^{-}, g_{f}^{\diamond}, g_{f}^{+}, g_{b}^{-}, g_{b}^{\diamond-}, g_{b}^{\diamond+}, g_{b}^{+}\right) \in \mathcal{H}_{\mathrm{qs}}
$$

and any pair of boundary values $\left(\phi_{\mathrm{hw}}, \vartheta_{\mathrm{hw}}\right) \in C([-1,1], \mathbb{C}) \times \mathbb{C}$, the set of functions

$$
h_{\mathrm{qs}}=L_{\mathrm{qs}}(\omega)\left(g_{\mathrm{qs}}, \phi_{\mathrm{hw}}, \vartheta_{\mathrm{hw}}\right) \in \mathcal{H}_{\mathrm{qs}}^{\circ} \text {, }
$$

written as (6.51), is the unique set that satisfies the following properties.

(i) The linear system

$$
\Lambda_{\mathrm{qsf}}^{\#}(\omega)\left(v_{f}^{\#}, \theta_{f}^{\#}\right)=\left(-\theta_{f}^{\#}, 0\right)+g_{f}^{\#}
$$

is satisfied for $\#=-, \diamond,+$. In addition, the linear system

$$
\Lambda_{\mathrm{qsb}}^{\#}(\omega)\left(v_{b}^{\#}, \theta_{b}^{\#}\right)=\left(-\theta_{b}^{\#}, 0\right)+g_{b}^{\#}
$$

is satisfied for $\#=-, \diamond-, \diamond+,+$.

(ii) The continuity conditions $\theta_{f}^{\diamond}(0)=\theta_{f}^{-}(0), \theta_{f}^{+}\left(\xi_{0}\right)=\theta_{f}^{\diamond}\left(\xi_{0}\right)$, together with $\theta_{b}^{\diamond-}\left(-\xi_{0}\right)=\theta_{b}^{-}\left(-\xi_{0}\right), \theta_{b}^{\diamond+}(0)=\theta_{b}^{\diamond-}(0)$ and $\theta_{b}^{+}\left(\xi_{0}\right)=\theta_{b}^{\diamond+}\left(\xi_{0}\right)$, all hold.

(iii) The continuity conditions

$$
\mathrm{ev}_{\xi_{0}} v_{f}^{\diamond}=\mathrm{ev}_{\xi_{0}} v_{f}^{+}, \quad \mathrm{ev}_{-\xi_{0}} v_{b}^{-}=\mathrm{ev}_{-\xi_{0}} v_{b}^{\diamond-} \quad \text { and } \quad \mathrm{ev}_{\xi_{0}} v_{b}^{\diamond+}=\mathrm{ev}_{\xi_{0}} v_{b}^{+}
$$

all hold.

(iv) We have the inclusions

$$
\begin{aligned}
& \mathrm{ev}_{0} e_{2 \eta} v_{f}^{-}, \mathrm{ev}_{0} e_{2 \eta} v_{f}^{\diamond} \in \Gamma_{f} \oplus \widehat{P}_{f} \oplus \widehat{Q}_{f}, \\
& \mathrm{ev}_{0} e_{2 \eta} v_{b}^{\diamond-}, \mathrm{ev}_{0} e_{2 \eta} v_{b}^{\diamond+} \in \Gamma_{b} \oplus \widehat{P}_{b} \oplus \widehat{Q}_{b} .
\end{aligned}
$$


(v) We have the jump conditions

$$
\begin{aligned}
& \mathrm{ev}_{0}\left[e_{2 \eta} v_{f}^{-}-e_{2 \eta} v_{f}^{\diamond}\right] \in \Gamma_{f}, \\
& \operatorname{ev}_{0}\left[e_{2 \eta} v_{b}^{\diamond-}-e_{2 \eta} v_{f}^{\diamond+}\right] \in \Gamma_{b} .
\end{aligned}
$$

(vi) We have the boundary conditions

$$
\begin{aligned}
& \mathrm{ev}_{\frac{1}{2} T} v_{f}^{+}-\mathrm{ev}_{-\frac{1}{2} T} v_{b}^{-}=\phi_{\mathrm{hw}}, \\
& \theta_{f}^{+}\left(\frac{1}{2} T\right)-\theta_{b}^{-}\left(-\frac{1}{2} T\right)=\vartheta_{\mathrm{hw}} .
\end{aligned}
$$

Furthermore, there exists a constant $\nu>0$ of order $\nu=O\left(\left(\xi_{*} M_{T}\right)^{-1}\right)$ such that the map

$$
T^{\mathrm{sl}} \mapsto L_{\mathrm{qs}}\left(\xi_{0}, T^{\mathrm{sl}}, T\right) \in \mathcal{L}\left(\mathcal{H}_{\mathrm{qs}} \times C([-1,1], \mathbb{C}) \times \mathbb{C}, \mathcal{H}_{\mathrm{qs}, \nu}^{\circ}\right)
$$

is $C^{1}$-smooth for any $\left(\xi_{0}, T^{\mathrm{sl}}, T\right) \in \Omega$. In addition, for some $C>0$, we have the uniform estimates

$$
\begin{array}{ll}
\left\|L_{\mathrm{qs}}(\omega)\left(g_{\mathrm{qs}}, \phi_{\mathrm{hw}}, \vartheta_{\mathrm{hw}}\right)\right\|_{\mathcal{H}_{\mathrm{qs}}^{\circ}} & \leq C\left[\left\|g_{\mathrm{qs}}\right\|_{H_{\mathrm{qs}}}+e^{-\frac{1}{2} \eta T}\left\|\phi_{\mathrm{hw}}\right\|+e^{-\frac{1}{2} \eta T}\left|\vartheta_{\mathrm{hw}}\right|\right], \\
\left\|D_{T^{\mathrm{s}}} L_{\mathrm{qs}}(\omega)\left(g_{\mathrm{qs}}, \phi_{\mathrm{hw}}, \vartheta_{\mathrm{hw}}\right)\right\|_{\mathcal{H}_{\mathrm{qs}, \nu}^{\circ}} \leq C e^{\nu T}\left[\left\|g_{\mathrm{qs}}\right\|_{H_{\mathrm{qs}}}+e^{-\frac{1}{2} \eta T}\left\|\phi_{\mathrm{hw}}\right\|+e^{-\frac{1}{2} \eta T}\left|\vartheta_{\mathrm{hw}}\right|\right],
\end{array}
$$

which hold for all $\omega \in \Omega$.

Proof. Solving the linear systems in (i) for the $\theta$-variables under the continuity conditions in (ii) and the boundary condition in (vi), we obtain

$$
\begin{aligned}
\theta_{f}^{-}(\xi) & =e^{-2 \eta \xi} \frac{1}{c(\epsilon)} \int_{-\infty}^{\xi} e^{2 \eta \xi^{\prime}} g_{f, 2}^{-}\left(\xi^{\prime}\right) \mathrm{d} \xi^{\prime} \\
\theta_{f}^{\diamond}(\xi) & =\theta_{f}^{-}(0) e^{-2 \eta \xi}+\frac{1}{c(\epsilon)} e^{-2 \eta \xi} \int_{0}^{\xi} e^{2 \eta \xi^{\prime}} g_{f, 2}^{\diamond}\left(\xi^{\prime}\right) \mathrm{d} \xi^{\prime}, \\
\theta_{f}^{+}(\xi) & =\theta_{f}^{\diamond}\left(\xi_{0}\right) e^{-2 \eta\left(\xi-\xi_{0}\right)}+\frac{1}{c(\epsilon)} e^{-2 \eta \xi} \int_{\xi_{0}}^{\xi} e^{2 \eta \xi^{\prime}} g_{f, 2}^{+}\left(\xi^{\prime}\right) \mathrm{d} \xi^{\prime}, \\
\theta_{b}^{-}(\xi) & =\left[\vartheta_{\mathrm{hw}}+\theta_{f}^{+}\left(\xi_{0}+T\right)\right] e^{-2 \eta\left(\xi+\xi_{0}+T\right)}+\frac{1}{c(\epsilon)} e^{-2 \eta \xi} \int_{-\xi_{0}}^{\xi} e^{2 \eta \xi^{\prime}} g_{b, 2}^{-}\left(\xi^{\prime}\right) \mathrm{d} \xi^{\prime}, \\
\theta_{b}^{\diamond-}(\xi) & =\theta_{b}^{-}\left(-\xi_{0}\right) e^{-2 \eta\left(\xi+\xi_{0}\right)}+\frac{1}{c(\epsilon)} e^{-2 \eta \xi} \int_{0}^{\xi} e^{2 \eta \xi^{\prime}} g_{b, 2}^{\diamond-}\left(\xi^{\prime}\right) \mathrm{d} \xi^{\prime}, \\
\theta_{b}^{\diamond+}(\xi) & =\theta_{b}^{\diamond-}(0) e^{-2 \eta \xi}+\frac{1}{c(\epsilon)} e^{-2 \eta \xi} \int_{\xi_{0}}^{\xi} e^{2 \eta \xi^{\prime}} g_{b, 2}^{\diamond+}\left(\xi^{\prime}\right) \mathrm{d} \xi^{\prime}, \\
\theta_{b}^{+}(\xi) & =\theta_{b}^{\diamond+}\left(\xi_{0}\right) e^{-2 \eta\left(\xi-\xi_{0}\right)}+\frac{1}{c(\epsilon)} e^{-2 \eta \xi} \int_{-\infty}^{\xi} e^{2 \eta \xi^{\prime}} g_{b, 2}^{+}\left(\xi^{\prime}\right) \mathrm{d} \xi^{\prime} .
\end{aligned}
$$

To obtain the accompanying functions $\left(v_{f}^{-}, v_{f}^{\diamond}, v_{f}^{+}, v_{b}^{-}, v_{b}^{\diamond-}, v_{b}^{\diamond+}, v_{b}^{+}\right)$and complete the proof, we may now proceed precisely as in the proof of [HS10, Lem. 6.1].

In order to find the quadruplet $\left(u_{\mathrm{qsf}}, u_{\mathrm{qsx}}, u_{\mathrm{qsb}}, w_{\mathrm{qs}}\right)$ described in Proposition 5.6. we need to solve the fixed point problem

$$
\begin{aligned}
h_{\mathrm{qs}}=L_{\mathrm{qs}}(\omega)\left(-\lambda h_{\mathrm{qs}}+B_{\mathrm{qs}}(\omega, \lambda) h_{\mathrm{qs}}, 0,0\right) \\
\quad+L_{\mathrm{qs}}(\omega)\left(M_{\mathrm{qs}}(\omega, \lambda)\left(\tilde{\kappa}_{f}, \tilde{\kappa}_{b}\right), \Phi_{\mathrm{hw}}(\omega, \lambda)\left(\tilde{\kappa}_{f}, \tilde{\kappa}_{b}\right), \Theta_{\mathrm{hw}}(\omega, \lambda)\left(\tilde{\kappa}_{f}, \tilde{\kappa}_{b}\right)\right),
\end{aligned}
$$

in which we have

$$
\begin{aligned}
& \Phi_{\mathrm{hw}}(\omega, \lambda)\left(\tilde{\kappa}_{f}, \tilde{\kappa}_{b}\right)=\tilde{\kappa}_{b} \mathrm{ev}_{-\frac{1}{2} T} u_{\mathrm{qb}}(\omega, \lambda)-\tilde{\kappa}_{f} \mathrm{ev}_{\frac{1}{2} T} u_{\mathrm{qf}}(\omega, \lambda), \\
& \Theta_{\mathrm{hw}}(\omega, \lambda)\left(\tilde{\kappa}_{f}, \tilde{\kappa}_{b}\right)=\tilde{\kappa}_{b} w_{\mathrm{qb}}(\omega, \lambda)\left(-\frac{1}{2} T\right)-\tilde{\kappa}_{f} w_{\mathrm{qf}}(\omega, \lambda)\left(\frac{1}{2} T\right) .
\end{aligned}
$$


Proposition 5.5 implies that we have the estimates

$$
\begin{aligned}
\left\|\Phi_{\mathrm{hw}}(\omega, \lambda)\left(\tilde{\kappa}_{f}, \tilde{\kappa}_{b}\right)\right\|+ & \left|\Theta_{\mathrm{hw}}(\omega, \lambda)\left(\tilde{\kappa}_{f}, \tilde{\kappa}_{b}\right)\right| \\
& \leq C e^{-\frac{1}{2} \eta T}\left(\left|\tilde{\kappa}_{f}\right|+\left|\tilde{\kappa}_{b}\right|\right), \\
\left\|D_{\left(T^{\mathrm{s}}, T, \lambda\right)} \Phi_{\mathrm{hw}}(\omega, \lambda)\left(\tilde{\kappa}_{f}, \tilde{\kappa}_{b}\right)\right\|+\left|D_{\left(T^{\mathrm{sl}}, T, \lambda\right)} \Theta_{\mathrm{hw}}(\omega, \lambda)\left(\tilde{\kappa}_{f}, \tilde{\kappa}_{b}\right)\right| & \leq C e^{-\frac{1}{2} \eta T}\left(\left|\tilde{\kappa}_{f}\right|+\left|\tilde{\kappa}_{b}\right|\right),
\end{aligned}
$$

for some $C>0$. In view of the bound (6.60), the fixed point problem (6.76) can be solved to yield a map

$$
h_{\mathrm{qs}}^{*}(\omega, \lambda) \in \mathcal{L}\left(\mathbb{C}^{2}, \mathcal{H}_{\mathrm{qs}}^{\circ}\right) .
$$

Using (6.63) and (6.78) we find the estimate

$$
\begin{array}{ll}
\left\|h_{\mathrm{qs}}^{*}(\omega, \lambda)\left(\tilde{\kappa}_{f}, \tilde{\kappa}_{b}\right)\right\|_{\mathcal{H}_{\mathrm{qs}}^{\circ}} & \leq C e^{-\eta T}\left(\left|\tilde{\kappa}_{f}\right|+\left|\tilde{\kappa}_{b}\right|\right), \\
\left\|D_{\left(T^{\mathrm{sl}}, \lambda\right)} h_{\mathrm{qs}}^{*}(\omega, \lambda)\left(\tilde{\kappa}_{f}, \tilde{\kappa}_{b}\right)\right\|_{\mathcal{H}_{\mathrm{qs}, \nu}^{\circ}} & \leq C e^{\nu T} e^{-\eta T}\left(\left|\tilde{\kappa}_{f}\right|+\left|\tilde{\kappa}_{b}\right|\right) .
\end{array}
$$

The differentiability of the functions $\tilde{\chi}_{f}$ and $\widetilde{\chi}_{b}$ defined in (5.42) with respect to the remaining variable $T$ can be obtained similarly as in [HS10, §6]. This completes the proof of Proposition 5.6 .

6.3. Region $R_{2}$. We now set out to establish Proposition 5.9. In contrast to our approach for $R_{1}$, we will attempt to construct potential eigenfunctions in a direct fashion. In particular, we reuse the function spaces $\mathcal{H}_{\mathrm{qs}}$ and $\mathcal{H}_{\mathrm{qs}}^{\circ}$ and set out to characterize sets $h_{\mathrm{qs}} \in \mathcal{H}_{\mathrm{qs}}^{\circ}$ that yield solutions to (5.37) by joining the different segments together.

This means that we must solve the linear system

$$
\left[\Lambda_{\mathrm{qsf}}^{\#}(\omega)+\lambda\right]\left(v_{f}^{\#}, \theta_{f}^{\#}\right)=\left(-\theta_{f}^{\#}, 0\right)+B_{\mathrm{qsf}}^{\#}(\omega, \lambda)\left(v_{f}^{\#}, \theta_{f}^{\#}\right)
$$

for $\#=-, \diamond,+$, together with

$$
\left[\Lambda_{\mathrm{qsb}}^{\#}(\omega)+\lambda\right]\left(v_{b}^{\#}, \theta_{b}^{\#}\right)=\left(-\theta_{b}^{\#}, 0\right)+B_{\mathrm{qsb}}^{\#}(\omega, \lambda)\left(v_{b}^{\#}, \theta_{b}^{\#}\right)
$$

for $\#=-, \diamond-, \diamond+,+$, in which all the operators are the same as in $\$ 6.2$ We obtain the following result.

Lemma 6.3. Pick $\delta_{0}>0$ to be sufficiently small and consider any $\lambda_{0} \notin 2 \pi i c_{*} \mathbb{Z}$ that has $\operatorname{Re} \lambda \geq-\delta_{0}$. Then there exist large constants $\xi_{*}>0, M_{T}>0$ and a small constant $\delta_{\mathrm{sl}}>0$ such that the following holds true. For any $\omega=\left(\xi_{0}, T^{\mathrm{sl}}, T\right) \in$ $\Omega\left(\xi_{*}, \delta_{\mathrm{sl}}, M_{T}\right)$ there exists a linear map

$$
L_{\mathrm{qs}, \lambda_{0}}(\omega): \mathcal{H}_{\mathrm{qs}} \rightarrow \mathcal{H}_{\mathrm{qs}}^{\circ}
$$

such that for any $g_{\mathrm{qs}} \in \mathcal{H}_{\mathrm{qs}}$, the set of functions

$$
h_{\mathrm{qs}}=L_{\mathrm{qs}, \lambda_{0}}(\omega)\left(g_{\mathrm{qs}}\right) \in \mathcal{H}_{\mathrm{qs}}^{\circ},
$$

written as in (6.51), is the unique set that satisfies the following properties.

(i) The linear system

$$
\left[\Lambda_{\mathrm{qsf}}^{\#}(\omega)+\lambda_{0}\right]\left(v_{f}^{\#}, \theta_{f}^{\#}\right)=\left(-\theta_{f}^{\#}, 0\right)+g_{f}^{\#}
$$

is satisfied for $\#=-, \diamond,+$. In addition, the linear system

$$
\left[\Lambda_{\mathrm{qsb}}^{\#}(\omega)+\lambda_{0}\right]\left(v_{b}^{\#}, \theta_{b}^{\#}\right)=\left(-\theta_{b}^{\#}, 0\right)+g_{b}^{\#}
$$

is satisfied for $\#=-, \diamond-, \diamond+,+$. 
(ii) The following continuity conditions all hold:

$$
\begin{array}{llll}
\mathrm{ev}_{0} v_{f}^{-} & =\mathrm{ev}_{0} v_{f}^{\diamond}, & \theta_{f}^{-}(0) & =\theta_{f}^{\diamond}(0), \\
\mathrm{ev}_{\xi_{0}} v_{f}^{\diamond}=\mathrm{ev}_{\xi_{0}} v_{f}^{+}, & \theta_{f}^{\diamond}\left(\xi_{0}\right) & =\theta_{f}^{+}\left(\xi_{0}\right), \\
\mathrm{ev}_{\frac{1}{2}} v_{f}^{+} & =\mathrm{ev}_{-\frac{1}{2} T} v_{b}^{-}, & \theta_{f}^{+}\left(\frac{1}{2} T\right) & =\theta_{b}^{-}\left(-\frac{1}{2} T\right), \\
\mathrm{ev}_{-\xi_{0}} v_{b}^{-} & =\mathrm{ev}_{-} \xi_{0} v_{b}^{\diamond-}, & \theta_{b}^{-}\left(-\xi_{0}\right) & =\theta_{b}^{\diamond-}\left(-\xi_{0}\right), \\
\mathrm{ev}_{0} v_{b}^{\diamond-} & =\mathrm{ev}_{0} v_{b}^{\diamond+}, & \theta_{b}^{\diamond-}(0) & =\theta_{b}^{\diamond+}(0), \\
\mathrm{ev}_{\xi_{0}} v_{b}^{\diamond+} & =\mathrm{ev}_{\xi_{0}} v_{b}^{+}, & \theta_{b}^{\diamond+}\left(\xi_{0}\right) & =\theta_{b}^{+}\left(\xi_{0}\right) .
\end{array}
$$

This map $L_{\mathrm{qs}, \lambda_{0}}(\omega)$ can be bounded uniformly for $\omega \in \Omega$.

Proof. We may proceed as in the proof of Lemma 6.2. The only difference is that now

$$
C([-1,1], \mathbb{C})=P_{f, \lambda} \oplus Q_{f, \lambda},
$$

which means that we can solve the linear systems in (i) with the additional continuity conditions $\mathrm{ev}_{0} v_{f}^{-}=\mathrm{ev}_{0} v_{f}^{\diamond}$ and $\mathrm{ev}_{0} v_{b}^{\diamond-}=\mathrm{ev}_{0} v_{b}^{\diamond+}$.

Proof of Proposition 5.9. Let us first take $\lambda=\lambda_{0}$. The differential equation (5.37) admits a nontrivial solution if and only if

$$
h_{\mathrm{qs}}=L_{\mathrm{qs}, \lambda_{0}}(\omega) B\left(\omega, \lambda_{0}\right) h_{\mathrm{qs}}
$$

admits a nontrivial solution. In view of the estimate (6.60), this can be prevented by choosing $\xi_{*}, M_{T}$ and $\delta_{\mathrm{sl}}$ appropriately. After taking $\delta_{\lambda}>0$ to be sufficiently small, the same argument works for all $\lambda \in \mathbb{C}$ that have $\left|\lambda-\lambda_{0}\right|<\delta_{\lambda}$.

\section{Discussion}

In this paper, we considered the discrete FitzHugh-Nagumo equation

$$
\begin{aligned}
& \dot{u}_{j}(t)=\alpha\left[u_{j+1}(t)-2 u_{j}(t)+u_{j-1}(t)\right]+g\left(u_{j}(t)\right)-w_{j}(t), \\
& \dot{w}_{j}(t)=\epsilon\left(u_{j}(t)-\gamma w_{j}(t)\right),
\end{aligned}
$$

posed on the one-dimensional lattice $j \in \mathbb{Z}$ with the cubic

$$
g(u)=g(u ; a)=u(1-u)(u-a) .
$$

We established that for all sufficiently small $\epsilon>0$, the travelling pulse

$$
\left(u_{j}, w_{j}\right)(t)=(\bar{u}(\epsilon), \bar{w}(\epsilon))(j+c(\epsilon) t)
$$

that was constructed in our previous paper HS10 is nonlinearly stable with an asymptotic phase. To prove this result, we considered the eigenvalue system

$$
\begin{aligned}
& c(\epsilon) u^{\prime}(\xi)=\alpha[u(\xi-1)+u(\xi+1)-2 u(\xi)]+g^{\prime}(\bar{u}(\epsilon)(\xi)) u(\xi)-w(\xi)-\lambda u(\xi), \\
& c(\epsilon) w^{\prime}(\xi)=\epsilon(u(\xi)-\gamma w(\xi))-\lambda w(\xi) .
\end{aligned}
$$

For an appropriate fixed choice of $0<\eta \ll 1$, we proved that (7.3) has, for each $\lambda$ near zero and each $0<\epsilon \ll 1$, a unique (up to normalization) solution of the form $(u, w)(\xi)=e^{2 \eta \xi}(\tilde{u}(\xi), \tilde{w}(\xi))$ such that $(\tilde{u}, \tilde{w})$ is bounded and piecewise differentiable with at most two jump discontinuities. These potential eigenfunctions $(u, w)(\xi)$ always decay to zero as $\xi \rightarrow-\infty$, and they also decay as $\xi \rightarrow \infty$ provided $\lambda$ is to the right of the essential spectrum associated with the pulse. If $\lambda$ lies to the left of the essential spectrum, the solutions $(u, w)(\xi)$ will, in general, grow exponentially 
as $\xi \rightarrow \infty$. Equations (5.44) and (5.45) show that the two jumps of $(u, w)$ vanish so that $(u, w)$ is a solution of (7.3) on $\mathbb{R}$, if and only if the linear system

$$
\begin{array}{cc}
\left(\begin{array}{cc}
\lambda M_{f, \lambda}+O\left(\lambda^{2}+\epsilon(|\epsilon|+|\lambda|)|\ln \epsilon|\right) & e^{-2 \eta T} O\left(e^{-\beta / \epsilon}\right) \\
e^{2 \eta T} O\left(e^{-\beta / \epsilon}\right) & \lambda M_{b, \lambda}+\epsilon M_{b, \epsilon}+O\left(\lambda^{2}+\epsilon(|\epsilon|+|\lambda|)|\ln \epsilon|\right)
\end{array}\right) \\
\times\left(\begin{array}{c}
\kappa_{f} \\
\kappa_{b}
\end{array}\right)=0 &
\end{array}
$$

has a nontrivial solution $\left(\kappa_{f}, \kappa_{b}\right)$, where $M_{f, \lambda}<0$ and $M_{b, \lambda}, M_{b, \epsilon}>0$ are the constants defined in (5.35) $, \beta, \eta>0$, and $T \geq \delta / \epsilon$ is the width of the pulse profile. Clearly, the determinant of (7.4) will vanish for precisely two values of $\lambda$ near zero: one of these roots is $\lambda=0$, which we know is an eigenvalue due to translational invariance of the travelling wave equation or, alternatively, from the fact that travelling waves are relative periodic orbits of the lattice system (7.1). The second root is given by

$$
\lambda_{2}(\epsilon)=-\frac{\epsilon M_{b, \epsilon}(1+o(1))}{M_{b, \lambda}},
$$

which lies in the open left half-plane. Since the rightmost part of the essential spectrum associated with the linearization about the travelling pulse, which is given in (5.7) by

$$
\lambda_{\text {ess }}(\nu, \epsilon)=-i \nu c-\epsilon\left(\gamma+\frac{1}{a+2 \alpha(1-\cos \nu)}\right)+O\left(\epsilon^{2}\right), \quad \nu \in \mathbb{R}
$$

also lies in the open left half-plane when $\epsilon>0$, the pulse is spectrally stable and therefore nonlinearly stable because of the results reviewed in $\$ 2$. Due to the decay properties of the solutions $(u, w)$ associated with $\lambda_{2}$ that we discussed above, we refer to $\lambda_{2}$ as an eigenvalue or a resonance pole depending on whether it lies respectively to the right or left of the essential spectrum.

We now comment on the interpretation of these results for the dynamics of the discrete FitzHugh-Nagumo equation (7.1) near the travelling pulse. Nonlinear
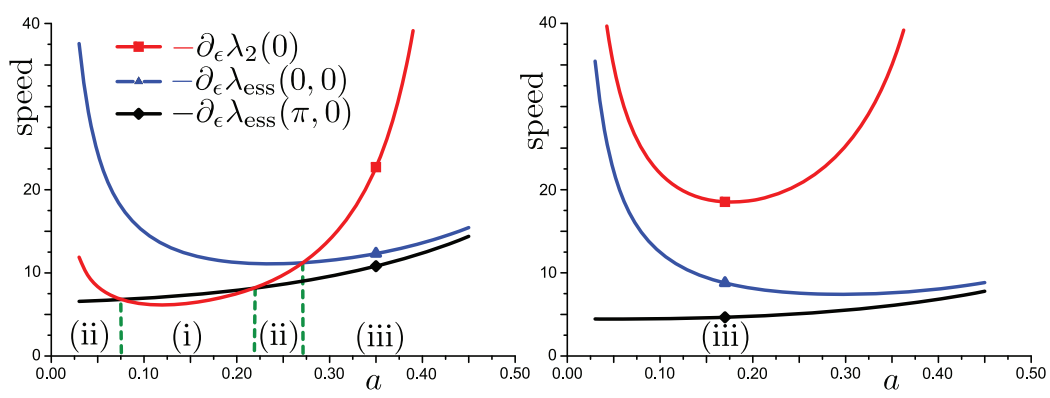

Figure 12. Plot of the speeds at which $\lambda_{2}$ and the essential spectrum move to the left at $\epsilon=0$. In both graphs, we used $\alpha=0.1$. In the left graph, we chose $\gamma=2(a-1)^{-2}$, while $\gamma=3.999(a-1)^{-2}$ in the right graph. The functions $q_{b}^{\prime}$ and $d_{b}$ appearing in (5.35) were computed using the numerical procedure described in HVL05. The labels (i), (ii) and (iii) correspond to the different cases shown in Figure 2, 
stability implies that small perturbations of the initial pulse profile will decay to zero exponentially as $t \rightarrow \infty$, though the final profile may experience a small phase shift. One interesting question is whether we can say more about how small perturbations decay to zero. This question is linked to the spectrum of the pulse that we discussed above, since the leading-order decay of perturbations should be governed by the spectral element closest to the imaginary axis. Thus, we first discuss whether $\lambda_{2}$ lies to the right or left of the curve $\lambda_{\text {ess }}$ of essential spectrum, and we refer to Figure 2 for the possible cases that may occur. Using (7.5) and (17.6), we see that $\lambda_{2}(\epsilon)$ and $\lambda_{\text {ess }}(\nu, \epsilon)$ satisfy

$$
\begin{array}{ll}
-\partial_{\epsilon} \lambda_{2}(0) & =M_{b, \lambda}^{-1} M_{b, \epsilon}, \\
-\partial_{\epsilon} \lambda_{\text {ess }}(0,0) & =\gamma+\frac{1}{a}, \\
-\partial_{\epsilon} \lambda_{\text {ess }}(\pi, 0) & =\gamma+\frac{1}{a+4 \alpha} .
\end{array}
$$

The location of $\lambda_{2}$ is therefore determined by the Melnikov integrals $M_{b, \epsilon}$ and $M_{b, \lambda}$ given in (5.35) that require knowledge of the Nagumo front and back. The latter are not known explicitly, and we therefore used the numerical method described in HVL05 to calculate them. The results are shown in Figure 12, In particular, we find that all three scenarios depicted in Figure 2 can be realized, depending on the values of $(a, \gamma)$. However, the situation in which $\lambda_{2}$ is a resonant pole seems to be prevalent.

If $\lambda_{2}$ lies to the right of the essential spectrum, our results tie in well with the intuition that the fast pulse could be considered as a bound state of the Nagumo front $q_{f}$ and the back $q_{b}$. Indeed, our analysis in Proposition 5.6 and the paragraphs following it show that the eigenfunction associated with $\lambda_{2}$ is found by gluing together $\kappa_{f} q_{f}^{\prime}$ and $\kappa_{b} q_{b}^{\prime}$, where the amplitudes $\left(\kappa_{f}, \kappa_{b}\right)$ satisfy (7.4) with $\lambda=\lambda_{2}$. In particular, it is easy to see that

$$
\left(\kappa_{f}, \kappa_{b}\right)=\left(\frac{1}{\epsilon} e^{-2 \eta T} O\left(e^{-\beta / \epsilon}\right), 1\right)
$$

so that the eigenfunction associated with $\lambda_{2}$ is centered on the back: if a perturbation excites only this eigenfunction, that is, if the perturbation changes the position of the back only, then the back will move back to its original position, with exponential rate $\lambda_{2}$, without interacting with the front. Other perturbations will lead to both a translation of the pulse profile and a movement of the back relative to the front.

If $\lambda_{2}$ does not lie to the right of the essential spectrum, the situation is less clear. On the linear level, the essential spectrum corresponds to modes that, relative to the pulse profile, transport localized perturbations of the background state $(u, w)=0$ to the right towards $j=\infty$. Thus, if $\lambda_{2}$ is an eigenvalue, and not a resonance pole, we might expect that its effect will still be felt as it affects the relative position of the front and the back that make up the pulse profile. We do not have much insight into what happens when $\lambda_{2}$ is a resonance pole.

One way of attacking both of these two remaining cases could be to aim for a nonlinear stability result in exponentially weighted spaces, using techniques similar to those developed in GS07. Alternatively, one might gain some insight by studying this problem for the FitzHugh-Nagumo PDE (1.11), using pointwise estimates via Green's functions. On the level of the Green's function, the effects of point and 
essential spectrum can be separated, and the question is then whether a similar analysis can be lifted to the nonlinear level for sufficiently localized perturbations.

\section{ACKNOWLEDGMENTS}

The first author acknowledges support from the Netherlands Organization for Scientific Research (NWO). The second author was supported in part by the NSF through grant DMS-0907904.

\section{REFERENCES}

[BC99] P. W. Bates and A. Chmaj, A Discrete Convolution Model for Phase Transitions, Arch. Rational Mech. Anal. 150 (1999), 281-305. MR.1741258 (2001c:82026)

[BG02] S. Benzoni-Gavage, Stability of Semi-Discrete Shock Profiles by Means of an Evans Function in Infinite Dimensions, J. Dynam. Diff. Equations 14 (2002), 613-674. MR 1917653 (2003j:35204)

[BGHR03] S. Benzoni-Gavage, P. Huot, and F. Rousset, Nonlinear Stability of Semidiscrete Shock Waves, SIAM J. Math. Anal. 35 (2003), 639-707. MR2048404 (2005j:35147)

[BHSZ10] M. Beck, H. J. Hupkes, B. Sandstede, and K. Zumbrun, Nonlinear Stability of Semidiscrete Shocks for Two-Sided Schemes, SIAM J. on Mathematical Analysis 42 (2010), 857-903. MR2644362 (2011d:35301)

[Cah60] J. W. Cahn, Theory of Crystal Growth and Interface Motion in Crystalline Materials, Acta Met. 8 (1960), 554-562.

[Car77] G. Carpenter, A Geometric Approach to Singular Perturbation Problems with Applications to Nerve Impulse Equations, J. Diff. Eq. 23 (1977), 335-367. MR0442379 (56:762a)

[Car05a] A. Carpio, Asymptotic Construction of Pulses in the Discrete Hodgkin-Huxley Model for Myelinated Nerves, Physical Review E 72 (2005), 011905. MR2178366 (2006e:92008)

[Car05b] Wave Trains, Self-Oscillations and Synchronization in Discrete Media, Physica D 207 (2005), 117-136. MR2166978 (2006d:37165)

[CB02] A. Carpio and L. L. Bonilla, Pulse Propagation in Discrete Systems of Coupled Excitable Cells, SIAM J. Appl. Math. 63 (2002), 619-635. MR1951953 (2003m:92027)

[CH99] X. Chen and S. P. Hastings, Pulse Waves for a Semi-Discrete Morris-Lecar Type Model, J. Math. Bio. 38 (1999), 1-20. MR.1674025 (2000b:92006)

[CMPS98] S. N. Chow, J. Mallet-Paret, and W. Shen, Traveling Waves in Lattice Dynamical Systems, J. Diff. Eq. 149 (1998), 248-291. MR1646240(2000b:37092)

[CR93] L. O. Chua and T. Roska, The CNN paradigm, IEEE Trans. Circ. Syst. 40 (1993), $147-156$

[EN93] T. Erneux and G. Nicolis, Propagating Waves in Discrete Bistable Reaction-Diffusion Systems, Physica D 67 (1993), 237-244. MR1234443 (94e:92009)

[Eva75] J. W. Evans, Nerve Axon Equations, IV: The Stable and Unstable Impulse, Indiana Univ. Math. J. 24 (1975), 1169-1190. MR0393891 (52:14698)

[EVV05] C. E. Elmer and E. S. Van Vleck, Spatially Discrete FitzHugh-Nagumo Equations, SIAM J. Appl. Math. 65 (2005), 1153-1174. MR2147323 (2006a:35167)

[GS07] A. Ghazaryan and B. Sandstede, Nonlinear Convective Instability of Turing-Unstable Fronts Near Onset: A Case Study, SIAM J. Appl. Dyn. Sys. 6 (2007), 319-347. MR 2318657 (2008d:37137)

[Has76] S. Hastings, On Travelling Wave Solutions of the Hodgkin-Huxley Equations, Arch. Rat. Mech. Anal. 60 (1976), 229-257. MR0402302 (53:6123)

[Hen81] D. Henry, Geometric Theory of Semilinear Parabolic Equations, Lecture Notes in Mathematics, vol. 840, Springer-Verlag, Berlin, 1981. MR610244 (83j:35084)

[HHK52] A. L. Hodgkin, A. F. Huxley, and B. Katz, Measurement of Current-Voltage Relations in the Membrane of the Giant Axon of Loligo, J. Physiology 116 (1952), 424-448.

[HS49] A. F. Huxley and R. Stamplli, Evidence for Saltatory Conduction in Peripheral Meylinated Nerve Fibres, J. Physiology 108 (1949), 315-339.

[HS10] H. J. Hupkes and B. Sandstede, Travelling Pulse Solutions for the Discrete FitzHughNagumo System, SIAM J. Appl. Dyn. Sys. 9 (2010), 827-882. MR2665452 
[HVL93] J. K. Hale and S. M. Verduyn-Lunel, Introduction to Functional-Differential Equations, Springer-Verlag, New York, 1993. MR1243878 (94m:34169)

[HVL05] H. J. Hupkes and S. M. Verduyn-Lunel, Analysis of Newton's Method to Compute Travelling Waves in Discrete Media, J. Dyn. Diff. Eq. 17 (2005), 523-572. MR2165558 (2006e:65129)

[HVL07] Center Manifold Theory for Functional Differential Equations of Mixed Type, J. Dyn. Diff. Eq. 19 (2007), 497-560. MR2333418 (2008c:34156)

[HVL09] , Lin's Method and Homoclinic Bifurcations for Functional Differential Equations of Mixed Type, Indiana Univ. Math. J. 58 (2009), 2433-2487. MR2603755 (2011d:34147)

[HZ93] D. Hankerson and B. Zinner, Wavefronts for a Cooperative Tridiagonal System of Differential Equations, J. Dyn. Diff. Eq. 5 (1993), 359-373. MR.1223452 (94c:34120)

[JKL91] C. K. R. T. Jones, N. Kopell, and R. Langer, Construction of the FitzHugh-Nagumo Pulse Using Differential Forms, Patterns and Dynamics in Reactive Media (New York) (H. Swinney, G. Aris, and D. G. Aronson, eds.), IMA Volumes in Mathematics and its Applications, vol. 37, Springer, 1991, pp. 101-116. MR.1228919 (94e:92015)

[Jon84] C. K. R. T. Jones, Stability of the Travelling Wave Solutions of the FitzHugh-Nagumo System, Trans. AMS 286 (1984), 431-469. MR760971 (86b:35011)

[Kee87] J. P. Keener, Propagation and its Failure in Coupled Systems of Discrete Excitable Cells, SIAM J. Appl. Math. 47 (1987), 556-572. MR889639(88k:92028)

[KS98] J. Keener and J. Sneyd, Mathematical Physiology, Springer-Verlag, New York, 1998. MR 1673204 (2000c:92010)

[LE92] J. P. Laplante and T. Erneux, Propagation Failure in Arrays of Coupled Bistable Chemical Reactors, J. Phys. Chem. 96 (1992), 4931-4934.

[Lil25] R. S. Lillie, Factors Affecting Transmission and Recovery in the Passive Iron Nerve Model, J. of General Physiology 7 (1925), 473-507.

[MP99a] J. Mallet-Paret, The Fredholm Alternative for Functional Differential Equations of Mixed Type, J. Dyn. Diff. Eq. 11 (1999), 1-48. MR1680463 (2000j:34107)

[MP99b] J. Mallet-Paret, The Global Structure of Traveling Waves in Spatially Discrete Dynamical Systems, J. Dyn. Diff. Eq. 11 (1999), 49-128. MR1680459(2000k:37125)

[MPVL] J. Mallet-Paret and S. M. Verduyn-Lunel, Exponential Dichotomies and Wiener-Hopf Factorizations for Mixed-Type Functional Differential Equations, J. Diff. Eq., to appear.

[Ran78] L. A. Ranvier, Lećons sur l'histologie du système nerveux, par m. l. ranvier, recueillies par m. ed. weber, F. Savy, Paris, 1878.

[Rus89] A. Rustichini, Functional Differential Equations of Mixed Type: The Linear Autonomous Case, J. Dyn. Diff. Eq. 11 (1989), 121-143. MR1010963 (91b:34108)

[Ton03] A. Tonnelier, McKean Caricature of the FitzHugh-Nagumo Model: Traveling Pulses in a Discrete Diffusive Medium, Physical Review E 67 (2003), 036105. MR.1976825 (2004c:35228)

[Yan85] E. Yanagida, Stability of Fast Travelling Wave Solutions of the FitzHugh-Nagumo Equations, J. Math. Biol. 22 (1985), 81-104. MR802737 (87a:92019)

[Zin91] B. Zinner, Stability of Traveling Wavefronts for the Discrete Nagumo Equation, SIAM J. Math. Anal. 22 (1991), 1016-1020. MR1112063(92d:34146)

Division of Applied Mathematics, Brown University, 182 George Street, Providence, RHODE ISLAND 02912

E-mail address: hjhupkes@dam.brown.edu

Current address: Mathematisch Instituut, Universiteit Leiden, P.O. Box 9512, 2300 RA Leiden, The Netherlands

E-mail address: hhupkes@math.leidenuniv.nl

Division of Applied Mathematics, Brown University, 182 George Street, Providence, RHode ISLAND 02912

E-mail address: bjorn_sandstede@brown.edu 